\title{
ROBUST CONTROL PROBLEMS OF VORTEX DYNAMICS IN SUPERCONDUCTING FILMS WITH GINZBURG-LANDAU COMPLEX SYSTEMS
}

\author{
AZIZ BELMILOUDI
}

Received 10 December 2004; Accepted 1 March 2005

We formulate and study robust control problems for a two-dimensional time-dependent Ginzburg-Landau model with Robin boundary conditions on phase-field parameter, which describes the phase transitions taking place in superconductor films with variable thickness. The objective of such study is to control the motion of vortices in the superconductor films by taking into account the influence of noises in data. Firstly, we introduce the perturbation problem of the nonlinear governing coupled system of equations (the deviation from the desired target). The existence and the uniqueness of the solution of the perturbation are proved as well as stability under mild assumptions. Afterwards, the robust control problems are formulated in the case when the control is in the external magnetic field and in the case when the control is in the initial condition of the vector potential. We show the existence of an optimal solution, and we also find necessary conditions for a saddle point optimality.

Copyright (C 2006 Aziz Belmiloudi. This is an open access article distributed under the Creative Commons Attribution License, which permits unrestricted use, distribution, and reproduction in any medium, provided the original work is properly cited.

\section{Introduction}

The aim of this contribution is the study of robust control problems to describe the phenomenon of vortex structure in the superconducting phase transitions, using the timedependent Ginzburg-Landau (TDGL) complex superconductivity model. Such model was derived by Gor'kov and Eliashberg in [28] from the microscopic BCS (BardeenCooper-Schieffer) theory [4] for a superconductor with paramagnetic impurities. It involves the real vector potential $U$ for the total magnetic field and a complex phase-field variable $\phi$ so that $|\phi|^{2}=\phi \bar{\phi}(\bar{\phi}$ is the complex conjugate of $\phi$ ) gives the relative density of the superconducting charge carriers (Cooper pairs of electrons), which varies between 0 in the normal phase and 1 in the superconducting phase. The need for $\phi$ to be complex is associated with the macroscopic quantum nature of superconductivity. Here we will be connected with the response of a superconducting material to an applied magnetic field under isothermal conditions below its critical temperature $T_{c}$ (the transition from 
normally conducting to superconducting is usually associated with critical temperature). The time evolution of $(U, \phi)$ is then governed by the following system:

$$
\begin{aligned}
& \eta \rho \frac{\partial \phi}{\partial t}-i \eta \kappa \operatorname{div}(\rho U) \phi+b(U) \cdot(\rho b(U))(\phi)+\rho G(\phi)=0 \quad \text { in } \mathscr{2}=\Omega \times(0, T), \\
& \rho \frac{\partial U}{\partial t}+\operatorname{curl}(\rho \operatorname{curl}(U))-\nabla(\operatorname{div}(\rho U))+\rho \mathscr{R}(b(U)(\phi) \bar{\phi})=\operatorname{curl}(\rho H) \quad \text { in } 2
\end{aligned}
$$

subjected to the Robin-type boundary conditions

$$
\frac{1}{\kappa^{2}} \frac{\partial \phi}{\partial n}=\mu \phi, \quad U \cdot \mathbf{n}=0, \quad \operatorname{curl}(U)=H \quad \text { in } \Sigma=\partial \Omega \times(0, T),
$$

and the initial conditions

$$
\phi(0)=\phi_{0}, \quad U(0)=U_{0} \quad \text { in } \Omega,
$$

where the operator $b$ (the covariant derivative) and the function $G$ are defined by: $b(U)=$ $((i / \kappa) \nabla+U), \bar{b}(U)=((-i / \kappa) \nabla+U)$ and $G(z)=\left(|z|^{2}-1\right) z$. The domain $\Omega$ is an open bounded domain in $\mathbb{R}^{2}$ with Lipschitz boundary $\partial \Omega, \mathbf{n}$ is the unit normal to the surface of the superconductor $\Gamma=\partial \Omega$ and $\mu$ is an arbitrary real number (the boundary condition is appropriate for the superconductor interface with vacuum or an insulator if $\mu=0$ and for the superconductor interface with normal metal if $\mu \neq 0)$. $\mathscr{R}(\cdot)$ (resp., $\mathscr{I}(\cdot)$ ) denotes the real part (resp., the imaginary part) of the quantity in $(\cdot)$ and curl denote the curl operators defined by (on the $(x, y)$-plane)

$$
\begin{aligned}
& \operatorname{curl}(\phi)=\left(\frac{\partial \phi}{\partial y},-\frac{\partial \phi}{\partial x}\right)^{T} \quad(\phi \text { is a scalar }) \\
& \operatorname{curl}(U)=\frac{\partial u_{2}}{\partial x}-\frac{\partial u_{1}}{\partial y} \quad\left(U=\left(u_{1}, u_{2}\right) \text { is a vector }\right) .
\end{aligned}
$$

$H$ is the applied magnetic field, $\eta$ is the nondimensional diffusivity and $\rho>0$ is a smooth function characterizing the vertical shape of the superconducting films and satisfying the following hypothesis

$$
\rho \in C^{1}(\bar{\Omega}) \quad \text { such that } \rho_{0} \leq \rho \leq \rho_{1} \text { and }|\nabla \rho| \leq \rho_{d}
$$

where $\left(\rho_{0}, \rho_{1}, \rho_{d}\right)$ are nonnegative constants.

The positive constant $\kappa$ is the Ginzburg-Landau parameter with $\kappa=\lambda / \xi$, where $\xi$ is a coherence length describing the size of thermodynamic fluctuations in the superconducting phase, and $\lambda$ is the London penetration depth describing the depth to which an external magnetic field can penetrate the superconductor. The parameter $\kappa$ determine the type of superconducting material: $\kappa<1 / \sqrt{2}$ describes type-I superconductors, $\kappa>1 / \sqrt{2}$ describes type-II superconductors. 


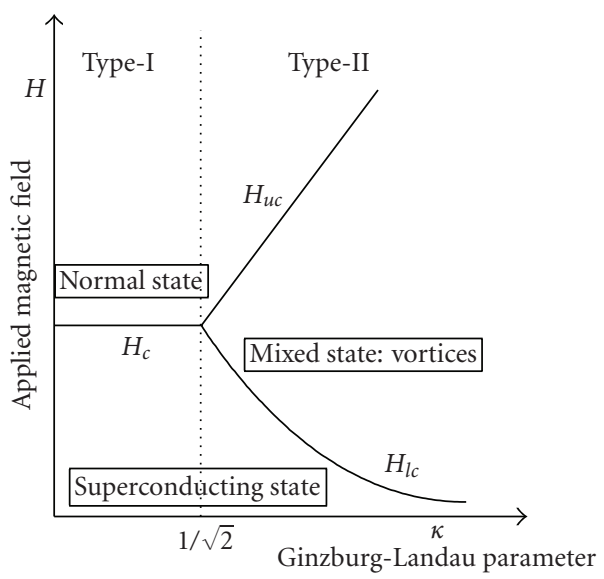

Figure 1.1. The reaction of a superconducting material.

Most applications of superconducting materials involve type-II superconductors in high magnetic fields. It is known that for the type-II superconductors, there is a critical magnetic field which splits into a lower critical field $H_{l c}$ and an upper critical field $H_{u c}$ (cf. Figure 1.1). For the magnetic fields below $H_{l c}$ the material is in the superconducting state and for magnetic field above $H_{u c}$ the material is in the normal state. For the magnetic fields between $H_{l c}$ and $H_{u c}$ the material is in the mixed state. This mixed state is described by physicists as follows: around some isolated points (called vortices, which are most commonly arranged in a hexagonal arrangement, see Abrikosov [1]) inside the material, the superconducting property is destroyed and the magnetic field become stronger in the nearby regions surrounding these vortices. While elsewhere, the superconducting property is still dominant and the field magnetic is excluded. Moreover, the motion of the vortices depends highly on the magnetization processes of the material (this is the result of the "Lorentz force" - that is more than anchorage forces of vortices and causes the displacement of the vortex-on the magnetic flux line carried by the vortex due to the transport current, e.g.). The motion of the vortices is undesirable, because this motion dissipate energy and leads to an electric field. So it is very interesting to study the applied magnetic in order to prevent their motion.

The objective of a robust control theory, which generalizes optimal control theory, is to compensate the undesirable effects of system disturbances through control actions such that a cost function achieves its minimum for the worst disturbances, that is, to find the best control which takes into account the worst-case (maximal) disturbance. More recently robust control frameworks have been the object of numerous studies either from a theoretical or from a numerical point of view to some classes of infinite (or finite)dimensional linear or nonlinear systems (see, e.g., [5-8, 10, 18, 24-26, 29, 31, 36, 37, 40] and the references therein).

Various problems associated with the Ginzburg-Landau models in superconductivity have been studied these last years (the literature on such model is vast, see, for example, $[9,11-13,15,17,19-22,30,38,39]$ and the references therein). For the optimal control 
problems associated with the TDGL models, we can mention [14] in which the authors studied the control of the vortices in superconducting films through the external magnetic field. Here, we consider a robust control problem, for the TDGL models with a Robin boundary condition on phase-field variable, which describes the phase transitions taking place in superconductors films, in order to take account the influence of noises in data. Indeed, such perturbations (noises) have the effect of impeding the ability of the material to become superconducting.

1.1. Assumptions and notations. We denote by $V_{n}=\left\{U \in H^{1}(\Omega) ; U \cdot \mathbf{n}=0\right.$ on $\left.\Omega\right\}$ and $V_{n}^{\prime}$ the dual of $V_{n}$. We denote by $\langle,\rangle_{V_{n}^{\prime}, V_{n}}$ the duality product between $V_{n}^{\prime}$ and $V_{n}$. For any pair of real number $r, s \geq 0$, we introduce the Sobolev space $H^{r, s}(2)$ defined by $H^{r, s}(2)=$ $L^{2}\left(0, T, H^{r}(\Omega)\right) \cap H^{s}\left(0, T, L^{2}(\Omega)\right)$, which is a Hilbert space normed by

$$
\left(\int_{0}^{T}\|v\|_{H^{r}(\Omega)}^{2} d t+\|v\|_{H^{s}\left(0, T, L^{2}(\Omega)\right)}^{2}\right)^{1 / 2},
$$

where $H^{s}\left(0, T, L^{2}(\Omega)\right)$ denotes the Sobolev space of order $s$ of functions defined on $(0, T)$ and taking values in $L^{2}(\Omega)$ and defined by $H^{s}\left(0, T, L^{2}(\Omega)\right)=\left[H^{m}\left(0, T, L^{2}(\Omega)\right), L^{2}(2)\right]_{\theta}$, where $\theta \in(0,1), s=(1-\theta) m, m$ is an integer and $H^{m}\left(0, T, L^{2}(\Omega)\right)=\left\{v \in L^{2}(2) \mid \partial^{j} v / \partial t^{j}\right.$ $\left.\in L^{2}(2), \forall j=1, m\right\}$.

Remark 1.1. (i) According to [27], we have the following embedding inequality on $V_{n}$ :

$$
\|U\|_{H^{1}}^{2} \leq C\left(\|U\|_{L^{2}}^{2}+\|\operatorname{div}(U)\|_{L^{2}}^{2}+\|\operatorname{curl}(U)\|_{L^{2}}^{2}\right), \quad \forall U \in V_{n} .
$$

(ii) For $v \in H^{r, s}(2)$, the trace functions of $v: \partial^{j} v / \partial n^{j}$ on $\Sigma=\partial \Omega \times(0, T)$ for an integer j such that $j \in[0, r-(1 / 2)]$ exist and satisfy $\partial^{j} v / \partial n^{j} \in H^{r_{j}, s_{j}}(\Sigma)$, where $r_{j}=r-j-1 / 2$ and $s_{j}=s(r-j-1 / 2) / r$. Moreover the functions $v \rightarrow \partial^{j} v / \partial n^{j}$ are continuous linear mappings from $H^{r, s}(2)$ into $H^{r_{j}, s_{j}}(\Sigma)$ (see, e.g., $[34,35]$ ).

If $X$ denotes some Banach space of real-valued functions, the corresponding space of complex-valued functions will be denoted by $\mathscr{X}$ and the corresponding space of vectorvalued functions, each of components belonging to $X$, will be denoted by $\mathbf{X}$, and we use $\|\cdot\|_{X}$ to denote the norms of spaces $X, \mathbf{X}$ or $\mathscr{L}$.

We can now introduce the following spaces: $\mathbf{W}_{n}=L^{2}\left(0, T, V_{n}\right) \cap H^{1}\left(0, T, V_{n}^{\prime}\right), \mathbf{E}_{n}=$ $L^{2}\left(0, T, V_{n}\right) \cap L^{\infty}\left(0, T, \mathbf{L}^{2}(\Omega)\right), \mathscr{W}=L^{2}\left(0, T, \mathscr{H}^{1}(\Omega)\right) \cap H^{1}\left(0, T,\left(\mathscr{H}^{1}\right)^{\prime}(\Omega)\right), \mathscr{E}=L^{2}(0, T$, $\left.\mathscr{H}^{1}(\Omega)\right) \cap L^{\infty}\left(0, T, \mathscr{L}^{2}(\Omega)\right)$ and $\mathscr{L}_{\infty}^{2}(\Omega)=\left\{\phi \in \mathscr{L}^{2}(\Omega) ;|\phi|\right.$ is bounded a.e. in $\left.\Omega\right\}$.

Remark 1.2. (i) $\mathcal{W}$ and $\mathbf{W}_{n}$ are continuously embedded into $C^{0}\left([0, T], \mathscr{L}^{2}(\Omega)\right)$ and $C^{0}([0$, $T], \mathbf{L}^{2}(\Omega)$ ), respectively (see, e.g., $[34,35]$ ).

(ii) Although $\mathscr{L}_{\infty}^{2}(\Omega)$ is a subset of $\mathscr{L}^{\infty}(\Omega)$, we equipped this space by the standard norm of the space $\mathscr{L}^{2}(\Omega)$. 
The weak formulation associated to problem (1.1) is then to find $(\phi, U) \in \mathcal{W} \times \mathbf{W}_{n}$ such that

$$
\begin{aligned}
& \eta \int_{\Omega} \rho \frac{\partial \phi}{\partial t} q d x-i \eta \kappa \int_{\Omega} \operatorname{div}(\rho U) \phi q d x+\int_{\Omega} \rho b(U)(\phi) \bar{b}(U)(q) d x \\
& \quad-i \mu \int_{\Gamma} \rho \phi q d \Gamma+\int_{\Omega} \rho G(\phi) q d x=0, \quad \text { a.e. } t \in(0, T), \forall q \in \mathcal{H}^{1}(\Omega), \\
& \int_{\Omega} \rho \frac{\partial U}{\partial t} v d x+\int_{\Omega} \rho \operatorname{curl}(U) \operatorname{curl}(v) d x+\int_{\Omega} \operatorname{div}(\rho U) \operatorname{div}(v) d x \\
& \quad+\int_{\Omega} \rho \mathscr{R}(b(U)(\phi) \bar{\phi}) v d x=\int_{\Omega} \rho H \operatorname{curl}(v) d x, \quad \text { a.e. } t \in(0, T), \forall v \in V_{n}, \\
& \phi(0)=\phi_{0}, \quad U(0)=U_{0} \quad \text { in } \Omega .
\end{aligned}
$$

\subsection{Preliminary results}

Definition 1.3. Let $\mathscr{K}_{1}$ and $\mathscr{K}_{2}$ be two arbitrary sets (whose nature will be stated precisely at each situation) and denote $\mathscr{K}=\mathscr{K}_{1} \times \mathscr{K}_{2}$.

A pair $\left(f^{*}, g^{*}\right) \in \mathscr{K}$ is a saddle point of the cost function $J$ on $\mathscr{K}$ if

$$
J\left(f^{*}, g\right) \leq J\left(f^{*}, g^{*}\right) \leq J\left(f, g^{*}\right), \quad \forall(f, g) \in \mathcal{K} .
$$

Lemma 1.4. Let $\Omega \subset \mathbb{R}^{m}, m \geq 1$, be an open and bounded set with a smooth boundary and let $q$ be a nonnegative integer. There exist the following results.

(i) $H^{q}(\Omega) \subset L^{p}(\Omega)$, for all $p \in[1,2 m /(m-2 q)]$, with continuous embedding (with the exception that if $2 q=m$, then $p \in[1,+\infty[$ and if $2 q>m$, then $p \in[1,+\infty])$.

(ii) (Gagliardo-Nirenberg inequalities). There exists $C>0$ such that $\|v\|_{L^{p}} \leq$ $C\|v\|_{H^{q}}^{\theta}\|v\|_{L^{2}}^{1-\theta}$, for all $v \in H^{q}(\Omega)$, where $0 \leq \theta<1$ and $p=2 m /(m-2 \theta q)$ (with the exception that if $q-m / 2$ is a nonnegative integer, then $\theta$ is restricted to 0$)$.

For the proof of this lemma, see for example Adams [2].

Lemma 1.5. (i) For all $z \in \mathbb{C}, G(z) \bar{z} \in \mathbb{R}$.

(ii) For all $\left(z_{1}, z_{2}\right) \in \mathbb{C}^{2}, G\left(z_{1}\right)-G\left(z_{2}\right)=\left(\left|z_{1}\right|^{2}+\left|z_{2}\right|^{2}-1\right)\left(z_{1}-z_{2}\right)+z_{1} z_{2}\left(\overline{z_{1}-z_{2}}\right)$.

Lemma 1.6. For $(\varphi, u)$ and $(\psi, v)$ sufficiently regular,

(i) $b(u)(\varphi)=b(v)(\varphi)+(u-v) \varphi$;

(ii) $b(u)(\varphi)-b(u)(\psi)=b(u)(\varphi-\psi)$;

(iii) $b(u)(\varphi) \cdot \overline{b(u)(\varphi)}=\left(1 / \kappa^{2}\right)|\nabla \varphi|^{2}+|u|^{2}|\varphi|^{2}-(2 / \kappa) \mathscr{S}(\bar{\varphi} \nabla \varphi) \cdot u$.

The proof of the previous lemmas are immediate.

Lemma 1.7. For $(u, v, w, X)$ sufficiently regular,

(i) $\|u\|_{H^{1}}\|v\|_{L^{4}}\|X\|_{L^{4}} \leq C_{1}\|u\|_{H^{1}}^{2}\|v\|_{L^{2}}+\delta\|\nabla X\|_{L^{2}}^{2}+C_{2}\left(\|v\|_{H^{1}}+\|v\|_{H^{1}}^{2}\right)\|X\|_{L^{2}}^{2}$, with $\delta$ chooses suitably at each situation,

(ii) $\|u\|_{L^{4}}\|v\|_{L^{4}}\|w\|_{L^{4}}\|X\|_{L^{4}} \leq C_{1}\|u\|_{L^{4}}^{2}\|v\|_{L^{4}}^{2}+\gamma\|\nabla X\|_{L^{2}}^{2}+C_{2}\left(\|w\|_{L^{4}}^{4}+\|w\|_{L^{4}}^{2}\right)\|X\|_{L^{2}}^{2}$, with $\gamma$ chooses suitably at each situation. 
Proof. (i) By using Gagliardo-Nirenberg inequalities (Lemma 1.4, with $m=2$ ), we obtain $\|u\|_{H^{1}}\|v\|_{L^{4}}\|X\|_{L^{4}} \leq c_{1}\|u\|_{H^{1}}^{2}\|v\|_{L^{2}}+c_{2}\|v\|_{H^{1}}\|X\|_{L^{2}}^{2}+c_{3}\|v\|_{H^{1}}\|X\|_{L^{2}}\|\nabla X\|_{L^{2}}$ and then by using the Young's formula, we can deduce the result (i).

(ii) $\|u\|_{L^{4}}\|v\|_{L^{4}}\|w\|_{L^{4}}\|X\|_{L^{4}} \leq c_{1}\|u\|_{L^{4}}^{2}\|v\|_{L^{4}}^{2}+c_{2}\|w\|_{L^{4}}^{2}\|X\|_{L^{4}}^{2}$.

By using Gagliardo-Nirenberg inequalities, we can deduce that $\|w\|_{L^{4}}^{2}\|X\|_{L^{4}}^{2} \leq$ $c_{3}\|w\|_{L^{4}}^{2}\|X\|_{L^{2}}^{2}+c_{4}\|w\|_{L^{4}}^{2}\|X\|_{L^{2}}\|\nabla X\|_{L^{2}}$.

According to Young's formula, we can deduce the result (ii).

1.3. Outline of paper. The rest of the paper is organized as follows. In Section 2, we prove the existence and uniqueness of the problem (1.1). In Section 3, we introduce the initial perturbation problem and prove the existence and the uniqueness of the perturbation and obtain a stability result. In Section 4, we study the Fréchet differentiability of the solution operator of the perturbation problem. This property is necessary to develop the robust control problem. In Section 5, we study the robust control problem corresponding to obtain the saddle point of the cost function $J$. The functional $J$ is depending on the disturbance (or noise), the control and the perturbation solution in the domain $\Omega$ over the time interval under consideration $[0, T]$. The robust problem is formulated in two cases of control: firstly the control is in the external magnetic field and the disturbance is in the external magnetic field or in the initial condition of the order parameter variable, secondly the control is in the initial condition of the vector potential and the disturbance is in the external magnetic field or in the initial condition of the order parameter variable. We prove the existence of an optimal solution (saddle point), to the robust control problem under consideration, and give necessary optimality conditions. The optimality system is corresponding to identify the gradient of the cost function that is necessary to develop a numerical computation in order to solve the robust control problem.

\section{Existence and uniqueness of the solution of the TDGL model}

The following results concern the existence and the uniqueness of the solution of the Ginzburg-Landau model with Robin-type boundary conditions on phase-field parameter (1.1).

Theorem 2.1. For any $\left(\phi_{0}, U_{0}\right) \in \mathscr{L}^{2}(\Omega) \times \mathbf{L}^{2}(\Omega)$ satisfying $\left|\phi_{0}\right| \leq 1$ a.e. in $\Omega$ and $H \in$ $L^{2}(2)$, there exists a unique solution $(\phi, U) \in \mathcal{W} \times \mathbf{W}_{n}$ of (1.6) satisfying $|\phi| \leq 1$ a.e. in 2 . Moreover, the following estimation exists:

$$
\|\phi\|_{W}^{2}+\|U\|_{W_{n}}^{2} \leq C\left(\|H\|_{L^{2}(2)}^{2}+\left\|\phi_{0}\right\|_{L^{2}(\Omega)}^{2}+\left\|U_{0}\right\|_{L^{2}(\Omega)}^{2}\right) .
$$

Proof. The proof of this result is obtained by using the same technique as in, for example, $[14,15]$. Here, we sketch only the proof of the existence by using the method of lines. The uniqueness is obtained by using the same technique as to prove the stability result in Theorem 3.1.

To begin with, we introduce the following semidiscretized approximation problem: let $N \geq 1$ be an integer, let $\tau=T / N$ be the time size, $t_{j}=j \tau$, for $j=0, \ldots, N$. The 
approximation problem is then to find $\left(\varphi_{j}, u_{j}\right) \in \mathscr{H}^{1}(\Omega) \times V_{n}, j=1, N$, such that

$$
\begin{aligned}
& \eta \int_{\Omega} \rho \frac{\phi_{j}-\varphi_{j-1}}{\tau} q d x-i \eta \kappa \int_{\Omega} \operatorname{div}\left(\rho U_{j}\right) \phi_{j} q d x+\int_{\Omega} \rho b\left(U_{j}\right)\left(\phi_{j}\right) \bar{b}\left(U_{j}\right)(q) d x \\
& \quad-i \mu \int_{\Gamma} \rho \phi_{j} q d \Gamma+\int_{\Omega} \rho G\left(\phi_{j}\right) q d x=0, \\
& \int_{\Omega} \rho \frac{U_{j}-U_{j-1}}{\tau} v d x+\int_{\Omega} \rho \mathscr{R}\left(\overline{\phi_{j-1}} b\left(U_{j-1}\right)\left(\phi_{j-1}\right)\right) v d x+\int_{\Omega} \rho \operatorname{curl}\left(U_{j}\right) \operatorname{curl}(v) d x \\
& +\int_{\Omega} \operatorname{div}\left(\rho U_{j}\right) \operatorname{div}(v) d x=\int_{\Omega} \rho H_{j} \operatorname{curl}(v) d x,
\end{aligned}
$$

where $\left(\phi_{0}, U_{0}\right)$ is given by $(1.6)$, and $H_{j}(\cdot)=(1 / \tau) \int_{t_{j-1}}^{t_{j}} H(\cdot, t) d t, j=1, N$.

We first notice that the first part and the second part of system (2.2) are independent of each other. The second part of (2.2) is a linear elliptic problem for $U_{j}$ with $\left(U_{j-1}, \phi_{j-1}\right)$ given by the previous step. By using the standard argument we obtain the existence and the uniqueness of $U_{j} \in V_{n}$. The first part of (2.2) is a semilinear elliptic problem with respect to $\phi_{j}$. By using the regularity of $\left(U_{j}, \phi_{j-1}\right)$ and a standard argument, we obtain the existence and the uniqueness of $\phi_{j}$.

Prove now, by using the maximum principal, that $\left|\phi_{j}\right| \leq 1, \forall j \geq 1$.

Let us consider the following notation: $r^{+}=\max (r, 0)$ and $r^{-}=(-r)^{+}$and then $r=$ $r^{+}-r^{-}$. Prove now that if $\left|\phi_{0}\right| \leq 1$ a.e. in $\Omega$ then $\left|\phi_{j}\right| \leq 1, \forall j \geq 1$ a.e. in $\Omega$. Suppose now that $\left|\phi_{j-1}\right| \leq 1$ a.e. in $\Omega$, and prove that $\left|\phi_{j}\right| \leq 1$ a.e. in $\Omega$. By choosing $q=\left(\left|\phi_{j}\right|^{2}-1\right)^{+} \bar{\phi}_{j}$ in the first part of (2.2) and by taking the real part we have then

$$
\begin{aligned}
\eta \int_{\Omega} \rho & \frac{\left(\left(\left|\phi_{j}\right|^{2}-1\right)^{+}\right)^{2}}{\tau} d x+\eta \int_{\Omega} \rho \frac{\left(\left|\phi_{j}\right|^{2}-1\right)^{+}}{\tau} d x-\eta \int_{\Omega} \rho \mathscr{R}\left(\overline{\phi_{j}} \phi_{j-1}\right) \frac{\left(\left|\phi_{j}\right|^{2}-1\right)^{+}}{\tau} d x \\
& +\int_{\Omega} \rho\left|b\left(U_{j}\right)\left(\phi_{j}\right)\right|^{2}\left(\left|\phi_{j}\right|^{2}-1\right)^{+} d x \\
& +\left.\left.\int_{\Omega^{+}} \frac{\rho}{2 \kappa^{2}}|\nabla| \phi_{j}\right|^{2}\right|^{2} d x+\int_{\Omega} \rho\left|\phi_{j}\right|^{2}\left(\left(\left|\phi_{j}\right|^{2}-1\right)^{+}\right)^{2} d x=0,
\end{aligned}
$$

where $\Omega^{+}=\left\{\left.x \in \Omega|| \phi_{j}\right|^{2}-1>0\right\}$. This implies (since $\left|\phi_{j-1}\right| \leq 1$ )

$$
\begin{gathered}
\eta \int_{\Omega} \rho \frac{\left(\left(\left|\phi_{j}\right|^{2}-1\right)^{+}\right)^{2}}{2 \tau} d x+\eta \int_{\Omega} \rho \frac{\left(\left|\phi_{j}\right|^{2}-1\right)^{+}}{2 \tau} d x+\int_{\Omega} \rho\left|b\left(U_{j}\right)\left(\phi_{j}\right)\right|^{2}\left(\left|\phi_{j}\right|^{2}-1\right)^{+} d x \\
+\left.\left.\int_{\Omega^{+}} \frac{\rho}{2 \kappa^{2}}|\nabla| \phi_{j}\right|^{2}\right|^{2} d x+\int_{\Omega} \rho\left|\phi_{j}\right|^{2}\left(\left(\left|\phi_{j}\right|^{2}-1\right)^{+}\right)^{2} d x \leq 0 .
\end{gathered}
$$

Therefore, we have that $\left|\phi_{j}\right| \leq 1$ a.e. in $\Omega$. 
Prove now some estimates. First, we take $(q, v)=\left(\overline{\phi_{j}}, U_{j}\right)$ in $(2.2)$, by using the uniform boundness of the sequence $\left(\phi_{j}\right)_{j \geq 1}$ and the discrete Gronwall's formula we obtain the following estimate

$$
\begin{aligned}
& \max _{j=1, n}\left(\left\|\phi_{j}\right\|_{L^{2}}^{2}+\left\|U_{j}\right\|_{L^{2}}^{2}\right)+\tau \sum_{j=1, N}\left(\left\|\nabla \phi_{j}\right\|_{L^{2}}^{2}+\left\|\operatorname{div}\left(U_{j}\right)\right\|_{L^{2}}^{2}+\left\|\operatorname{curl}\left(U_{j}\right)\right\|_{L^{2}}^{2}\right) \\
& \quad \leq C\left(\left\|\phi_{0}\right\|_{L^{2}}^{2}+\left\|U_{0}\right\|_{L^{2}}^{2}+\|H\|_{L^{2}(2)}^{2}\right) .
\end{aligned}
$$

By taking now $(q, v)=(1 / \tau)\left(\overline{\phi_{j}-\phi_{j-1}}, U_{j}-U_{j-1}\right)$ in $(2.2)$, by using the uniform boundness of the sequence $\left(\phi_{j}\right)_{j \geq 1}$ and the discrete Gronwall's formula, we have

$$
\begin{aligned}
\tau \sum_{j=1, N} & \left(\left\|\frac{1}{\tau}\left(\phi_{j}-\phi_{j-1}\right)\right\|_{L^{2}}^{2}+\left\|\frac{1}{\tau}\left(u_{j}-u_{j-1}\right)\right\|_{L^{2}}^{2}\right) \\
& +\sum_{j=1, N}\left(\left\|\nabla \phi_{j}\right\|_{L^{2}}^{2}+\left\|\operatorname{div}\left(U_{j}\right)\right\|_{L^{2}}^{2}+\left\|\operatorname{curl}\left(U_{j}\right)\right\|_{L^{2}}^{2}\right) \\
\leq & C\left(\left\|\phi_{0}\right\|_{L^{2}}^{2}+\left\|U_{0}\right\|_{L^{2}}^{2}+\|H\|_{L^{2}(2)}^{2}\right) .
\end{aligned}
$$

By using (2.2), Green's formula, the estimates (2.5), (2.6), the uniform boundness of $\left(\phi_{j}\right)_{j \geq 1}$, and Lemma 1.4 we obtain the following estimation:

$$
\begin{aligned}
& \sum_{j=1, N}\left(\left\|\frac{1}{\tau}\left(\phi_{j}-\varphi_{j-1}\right)\right\|_{\left(H^{1}(\Omega)\right)^{\prime}}^{2}+\left\|\frac{1}{\tau}\left(U_{j}-U_{j-1}\right)\right\|_{V_{n}^{\prime}}^{2}\right) \\
& \leq C\left(\left\|\phi_{0}\right\|_{L^{2}}^{2}+\left\|U_{0}\right\|_{L^{2}}^{2}+\|H\|_{L^{2}(2)}^{2}\right) .
\end{aligned}
$$

The proof of theorem can be completed by standard convergence arguments (see, e.g., [32]), by taking advantage of the above estimates and by using the continuous mapping from $\mathscr{H}^{1 / 2+s}(\Omega)$ into $\mathscr{L}^{2}(\Gamma), 0<s<1 / 2$, see, for example, [34, 35] (to pass to the limit in term $\left.\int_{\Gamma} \rho \phi_{j} q d \Gamma\right)$.

Remark 2.2. Throughout the paper, we suppose that the hypotheses of Theorem 2.1 are satisfied, to ensure that the solution of problem (1.1), is in $\left(\mathcal{W} \cap \mathscr{L}^{\infty}(2)\right) \times \mathbf{W}_{n}$.

\section{Studying the perturbation problem}

In the following, the solution $(\phi, U)$ of problem (1.1) will be treated as the target function. We are then interested in the robust regulation of the deviation of the problem from the desired target $(\phi, U)$. We analyze the full nonlinear equation which models large perturbations $(\varphi, u)$ to the target $(\phi, U)$. Hence we consider the following system (for $(\phi, U)$ 
given satisfying the regularity of Theorem 2.1):

$$
\begin{aligned}
\eta \rho \frac{\partial \varphi}{\partial t}- & i \eta \kappa \operatorname{div}(\rho(u+U)) \varphi-i \eta \kappa \operatorname{div}(\rho u) \phi+b(u+U) \cdot(\rho b(u+U))(\varphi) \\
& +(b(u+U) \cdot(\rho b(u+U))-b(U) \cdot(\rho b(U)))(\phi) \\
& +\rho(G(\phi+\varphi)-G(\phi))=0 \quad \text { in } 2, \\
\rho \frac{\partial v}{\partial t}+ & \operatorname{curl}(\rho \operatorname{curl}(u))-\nabla(\operatorname{div}(\rho u)) \\
& +\rho \mathscr{R}(b(u+U)(\varphi+\phi)(\bar{\varphi}+\bar{\phi})-b(U)(\phi) \bar{\phi})=\operatorname{curl}(\rho h) \quad \text { in } 2,
\end{aligned}
$$

subjected to the Robin-type boundary conditions

$$
\frac{1}{\kappa^{2}} \frac{\partial \varphi}{\partial n}=\mu \varphi, \quad u \cdot \mathbf{n}=0, \quad \operatorname{curl}(u)=h \quad \text { in } \Sigma,
$$

and the initial conditions

$$
\varphi(0)=\varphi_{0}, \quad u(0)=u_{0} \quad \text { in } \Omega
$$

If we set $F(\varphi)=G(\varphi+\phi)-G(\phi), B(u)=b(U+u)$ then (3.1) is reduced to

$$
\begin{aligned}
& \eta \rho \frac{\partial \varphi}{\partial t}-\operatorname{i\eta \kappa } \operatorname{div}(\rho(u+U)) \varphi-i \eta \kappa \operatorname{div}(\rho u) \phi \\
& \quad+B(u) \cdot(\rho B(u))(\varphi+\phi)+\rho F(\varphi)=B(0) \cdot(\rho B(0))(\phi) \quad \text { in } 2, \\
& \rho \frac{\partial u}{\partial t}+\operatorname{curl}(\rho \operatorname{curl}(u))-\nabla(\operatorname{div}(\rho u))+\rho \mathscr{R}(B(u)(\varphi+\phi)(\overline{\varphi+\phi})) \\
& =\rho \mathscr{R}(B(0)(\phi) \bar{\phi})+\operatorname{curl}(\rho h) \quad \text { in } 2,
\end{aligned}
$$

subjected to the Robin-type boundary conditions

$$
\frac{1}{\kappa^{2}} \frac{\partial \varphi}{\partial n}=\mu \varphi, \quad u \cdot \mathbf{n}=0, \quad \operatorname{curl}(u)=h \quad \text { in } \Sigma,
$$

and the initial conditions

$$
\varphi(0)=\varphi_{0}, \quad u(0)=u_{0} \quad \text { in } \Omega
$$

Now we give the weak formulation associated to problem (3.2).

Multiplying the first part of (3.2) by $q \in \mathscr{H}^{1}(\Omega)$ and the second part by $v \in V_{n}$ and integrating over $\Omega$ gives (according to the third part of (3.2)) the weak formulation 


$$
\begin{aligned}
& \eta \int_{\Omega} \rho \frac{\partial \varphi}{\partial t} q d x-i \eta \kappa \int_{\Omega} \operatorname{div}(\rho(u+U)) \varphi q d x-i \eta \kappa \int_{\Omega} \operatorname{div}(\rho u) \phi q d x-i \mu \int_{\Gamma} \rho \varphi q d \Gamma \\
& \quad+\int_{\Omega} \rho B(u)(\varphi+\phi) \bar{B}(u)(q) d x+\int_{\Omega} \rho F(\varphi) q d x=\int_{\Omega} \rho B(0)(\phi) \bar{B}(0)(q) d x \\
& \int_{\Omega} \rho \frac{\partial v}{\partial t} v d x+\int_{\Omega} \rho \operatorname{curl}(u) \operatorname{curl}(v) d x+\int_{\Omega} \operatorname{div}(\rho u) \operatorname{div}(v) d x \\
& \quad+\int_{\Omega} \rho \mathscr{R}((\bar{\varphi}+\bar{\phi}) B(u)(\varphi+\phi)) v d x=\int_{\Omega} \rho \mathscr{R}(\bar{\phi} B(0)(\phi)) v d x+\int_{\Omega} \rho h \operatorname{curl}(v) d x \\
& (\varphi(0), u(0))=\left(\varphi_{0}, u_{0}\right)
\end{aligned}
$$

3.1. The existence and the stability results. Now we show the existence of the solution to the problem (3.3).

Theorem 3.1. For any $\left(\varphi_{0}, u_{0}\right) \in \mathscr{L}_{\infty}^{2}(\Omega) \times \mathbf{L}^{2}(\Omega)$ and $h \in L^{2}(2)$, there exists $(\varphi, u) \in$ $\left(\mathscr{W} \cap \mathscr{L}^{\infty}(2)\right) \times \mathbf{W}_{n}$ solution of (3.3). Moreover, the following estimation exists:

$$
\|\varphi\|_{W}^{2}+\|u\|_{W_{n}}^{2} \leq C\left(\|h\|_{L^{2}(2)}^{2}+\left\|\varphi_{0}\right\|_{L^{2}(\Omega)}^{2}+\left\|u_{0}\right\|_{L^{2}(\Omega)}^{2}\right)
$$

Proof. The proof of this result is a consequence of the result of Theorem 2.1, so we omit the tedious details.

Next we will establish a stability result which gives us uniqueness of solution of (3.2) as a corollary.

Theorem 3.2. Let $\left(u_{01}, \varphi_{01}, h_{1}\right)$ and $\left(u_{02}, \varphi_{02}, h_{2}\right)$ be functions of $\mathbf{L}^{2}(\Omega) \times \mathscr{L}_{\infty}^{2}(\Omega) \times L^{2}(2)$. If $\left(u_{1}, \varphi_{1}\right) \in \mathbf{W}_{n} \times\left(\mathcal{W} \cap \mathscr{L}^{\infty}(2)\right)$ (resp., $\left.\left(u_{2}, \varphi_{2}\right) \in \mathbf{W}_{n} \times\left(\mathcal{W} \cap \mathscr{L}^{\infty}(2)\right)\right)$ is solution of (3.3) with $\left(\varphi_{01}, u_{01}, h_{1}\right)$ (resp., $\left.\left(\varphi_{02}, u_{02}, h_{1}\right)\right)$ the given data, then the following estimation exists:

$$
\|\varphi\|_{W}^{2}+\|u\|_{W_{n}}^{2} \leq C\left(\left\|\varphi_{0}\right\|_{L^{2}}^{2}+\left\|u_{0}\right\|_{L^{2}}^{2}+\|h\|_{L^{2}(2)}^{2}\right)
$$

where $\varphi=\varphi_{1}-\varphi_{2}, u=u_{1}-u_{2}, \varphi_{0}=\varphi_{01}-\varphi_{02}, u_{0}=u_{01}-u_{02}$, and $h=h_{1}-h_{2}$.

Proof. Let $\left(\varphi_{i}, u_{i}, \varphi_{0 i}, u_{0 i}, h_{i}\right)_{i=1,2}$ be two solutions of (3.3) with $\left(\varphi_{0 i}, u_{0 i}\right)_{i=1,2}$ the initial data respectively. We denote by $\varphi=\varphi_{1}-\varphi_{2}, u=u_{1}-u_{2}, \varphi_{0}=\varphi_{01}-\varphi_{02}, u_{0}=u_{01}-u_{02}$, and 
$h=h_{1}-h_{2}$. Then the couple $(\varphi, u)$ is solution of

$$
\begin{aligned}
& \eta \int_{\Omega} \rho \frac{\partial \varphi}{\partial t} q d x-i \eta \kappa\left(\int_{\Omega} \operatorname{div}\left(\rho U_{2}\right) \varphi q d x+\int_{\Omega} \operatorname{div}(\rho u) \phi_{1} q d x\right)-i \mu \int_{\Gamma} \rho \varphi q d \Gamma \\
& \quad+\int_{\Omega} \rho B\left(u_{1}\right)(\varphi) \bar{B}\left(u_{1}\right)(q) d x+\int_{\Omega} \rho u \phi_{2} \bar{B}\left(u_{2}\right)(q) d x \\
& \quad+\int_{\Omega} \rho B\left(u_{1}\right)\left(\phi_{2}\right) u q d x+\int_{\Omega} \rho\left(F\left(\varphi_{1}\right)-F\left(\varphi_{2}\right)\right) q d x=0, \\
& \int_{\Omega} \rho \frac{\partial u}{\partial t} v d x+\int_{\Omega} \rho \operatorname{curl}(u) \operatorname{curl}(v) d x+\int_{\Omega} \operatorname{div}(\rho u) \operatorname{div}(v) d x+\int_{\Omega} \rho \mathscr{R}\left(\bar{\varphi} B\left(u_{1}\right)\left(\phi_{1}\right)\right) v d x \\
& \quad+\int_{\Omega} \rho \mathscr{R}\left(\bar{\phi}_{2} B\left(u_{1}\right)(\varphi)\right) v d x+\int_{\Omega} u\left|\phi_{2}\right|^{2} v d x=\int_{\Omega} \rho h \operatorname{curl}(v) d x, \\
& (\varphi(0), u(0))=\left(\varphi_{0}, u_{0}\right),
\end{aligned}
$$

where $U_{i}=u_{i}+U$ and $\phi_{i}=\varphi_{i}+\phi$, for $i=1,2$ (according to the regularity of $\left(\varphi_{i}, u_{i}\right)$ and $(\phi, U)$, the couple $\left(\phi_{i}, U_{i}\right)$ is in $\left.\left(W \cap \mathscr{L}^{\infty}(2)\right) \times \mathbf{W}_{n}\right)$.

By choosing $(q, v)=(\bar{\varphi}, u)$ in (3.6) and taking the real part of the first part of the system, we have (according to Lemma 1.5 and to the expression of $F$ )

$$
\begin{aligned}
& \eta \int_{\Omega} \rho \frac{\partial|\varphi|^{2}}{2 \partial t} d x+\eta \kappa \int_{\Omega} \operatorname{div}(\rho u) \mathscr{I}\left(\phi_{1} \bar{\varphi}\right) d x+\int_{\Omega} \rho B\left(u_{1}\right)(\varphi) \overline{B\left(u_{1}\right)(\varphi)} d x \\
& \quad+\int_{\Omega} \rho u \mathscr{R}\left(\phi_{2} \overline{B\left(u_{2}\right)(\varphi)}\right) d x+\int_{\Omega} \rho u \mathscr{R}\left(B\left(u_{1}\right)\left(\phi_{2}\right) \bar{\varphi}\right) d x \\
& \quad+\int_{\Omega} \rho\left(\left|\phi_{1}\right|^{2}+\left|\phi_{2}\right|^{2}-1\right)|\varphi|^{2} d x+\int_{\Omega} \rho \mathscr{R}\left(\phi_{1} \phi_{2}(\bar{\varphi})^{2}\right) d x=0, \\
& \int_{\Omega} \rho \frac{\partial|u|^{2}}{2 \partial t} v d x+\int_{\Omega} \rho|\operatorname{curl}(u)|^{2} d x+\int_{\Omega} \operatorname{div}(\rho u) \operatorname{div}(u) d x+\int_{\Omega} \rho \mathscr{R}\left(\bar{\varphi} B\left(u_{1}\right)\left(\phi_{1}\right)\right) u d x \\
& \quad+\int_{\Omega} \rho \mathscr{R}\left(\bar{\phi}_{2} B\left(u_{1}\right)(\varphi)\right) u+\int_{\Omega}|u|^{2}\left|\phi_{2}\right|^{2} d x=\int_{\Omega} \rho h \operatorname{curl}(u) d x, \\
& (\varphi(0), u(0))=\left(\varphi_{0}, u_{0}\right)
\end{aligned}
$$

and then (according to hypothesis (1.3), Lemma 1.6 and to the regularity of $\phi,\left(\phi_{i}\right)_{i=1,2}$, that is, in $\left.\mathscr{L}^{\infty}(2)\right)$

$$
\begin{aligned}
\eta \rho_{0} \frac{d\|\varphi\|_{L^{2}}^{2}}{2 d t}+\frac{\rho_{0}}{\kappa^{2}}\|\nabla \varphi\|_{L^{2}}^{2} \\
\leq c_{1} \int_{\Omega}|\operatorname{div}(\rho u)||\varphi| d x+c_{2} \int_{\Omega}\left|U_{1}\right||\varphi||\nabla \varphi| d x+c_{3} \int_{\Omega}\left(1+\left|U_{1}\right|^{2}\right)|\varphi|^{2} d x \\
\quad+c_{4} \int_{\Omega}\left(\left|\nabla \phi_{2}\right|+\left|U_{2}\right|+\left|U_{1}\right|\right)|\varphi||u| d x+c_{5} \int_{\Omega}|\nabla \varphi||u| d x,
\end{aligned}
$$


12 Robust control of Ginzburg-Landau models

$$
\begin{aligned}
& \rho_{0} \frac{d\|u\|_{L^{2}}^{2}}{2 d t}+\rho_{0}\left(\|\operatorname{curl}(u)\|_{L^{2}}^{2}+\|\operatorname{div}(u)\|_{L^{2}}^{2}\right) \\
& \quad \leq c_{6}\left(\int_{\Omega}|u||\operatorname{div}(u)| d x+\int_{\Omega}|h||\operatorname{curl}(u)| d x\right)+c_{7} \int_{\Omega}|u||\varphi|\left(\left|\nabla \phi_{1}\right|+\left|U_{1}\right|\right) d x \\
& \quad+c_{8} \int_{\Omega}|u||\nabla \varphi| d x+c_{9} \int_{\Omega}|u|^{2} d x \\
& (\varphi(0), u(0))=\left(\varphi_{0}, u_{0}\right) .
\end{aligned}
$$

According to the regularity of $U_{i}, \phi_{i}$, and by using Young's inequality, we have then for all $\delta>0$ and $\gamma>0\left(\right.$ since $\left.H^{1} \subset L^{4}\right)$,

$$
\begin{aligned}
& \eta \rho_{0} \frac{d\|\varphi\|_{L^{2}}^{2}}{2 d t}+\frac{\rho_{0}}{\kappa^{2}}\|\nabla \varphi\|_{L^{2}}^{2} \\
& \leq \delta\left(\|\operatorname{curl}(u)\|_{L^{2}}^{2}+\|\operatorname{div}(u)\|_{L^{2}}^{2}\right)+\frac{\rho_{0}}{4 \kappa^{2}}\|\nabla \varphi\|_{L^{2}}^{2}+c_{10}\left\|U_{1}\right\|_{L^{4}}\|\varphi\|_{L^{4}}\|\nabla \varphi\|_{L^{2}} \\
& \quad+c_{11}\left(\left\|U_{1}\right\|_{L^{4}}^{2}+\left\|U_{2}\right\|_{L^{4}}^{2}\right)\|\varphi\|_{L^{4}}^{2}+c_{12}\|u\|_{L^{4}}\|\varphi\|_{L^{4}}\left\|\nabla \phi_{2}\right\|_{L^{2}}+c_{13}\left(\|\varphi\|_{L^{2}}^{2}+\|u\|_{L^{2}}^{2}\right), \\
& \rho_{0} \frac{d\|u\|_{L^{2}}^{2}}{2 d t}+\rho_{0}\left(\|\operatorname{curl}(u)\|_{L^{2}}^{2}+\|\operatorname{div}(u)\|_{L^{2}}^{2}\right) \\
& \leq \frac{\rho_{0}}{4}\left(\|\operatorname{curl}(u)\|_{L^{2}}^{2}+\|\operatorname{div}(u)\|_{L^{2}}^{2}\right)+\gamma\|\nabla \varphi\|_{L^{2}}^{2}+c_{14}\|h\|_{L^{2}}^{2} \\
& \quad+c_{15}\|u\|_{L^{4}}\|\varphi\|_{L^{4}}\left(\left\|\nabla \phi_{1}\right\|_{L^{2}}+\left\|U_{1}\right\|_{L^{2}}\right)+c_{16}\left(\|\varphi\|_{L^{2}}^{2}+\|u\|_{L^{2}}^{2}\right), \\
& (\varphi(0), u(0))=\left(\varphi_{0}, u_{0}\right) .
\end{aligned}
$$

By using Gagliardo-Nirenberg inequalities (Lemma 1.4), we have

$$
\begin{aligned}
& \frac{d}{d t}\left(\|\varphi\|_{L^{2}}^{2}+\|u\|_{L^{2}}^{2}\right)+\|\nabla \varphi\|_{L^{2}}^{2}+\left(\|\operatorname{curl}(u)\|_{L^{2}}^{2}+\|\operatorname{div}(u)\|_{L^{2}}^{2}\right) \\
& \leq c_{17}\|h\|_{L^{2}}^{2}+c_{18}\left(1+\left\|\nabla \phi_{1}\right\|_{L^{2}}^{2}+\left\|\nabla \phi_{2}\right\|_{L^{2}}^{2}\right)\left(\|\varphi\|_{L^{2}}^{2}+\|u\|_{L^{2}}^{2}\right) \\
& \quad+c_{19}\left(\left\|U_{1}\right\|_{L^{4}}^{4}+\left\|U_{2}\right\|_{L^{4}}^{4}+\left\|U_{1}\right\|_{L^{4}}^{2}+\left\|U_{2}\right\|_{L^{4}}^{2}\right)\|\varphi\|_{L^{2}}^{2}, \\
& (\varphi(0), u(0))=\left(\varphi_{0}, u_{0}\right) .
\end{aligned}
$$

Since $U_{i}, i=1,2$, are in $L^{\infty}\left(0, T, \mathbf{L}^{2}(\Omega)\right)$, by using again Gagliardo-Nirenberg inequalities, we can deduce that (by integrating over $(0, t)$ for $t \in(0, T)$ )

$$
\begin{aligned}
\|\varphi\|_{L^{2}}^{2}+ & \|u\|_{L^{2}}^{2}+\int_{0}^{t}\|\nabla \varphi\|_{L^{2}}^{2} d s+\int_{0}^{t}\left(\|\operatorname{curl}(u)\|_{L^{2}}^{2}+\|\operatorname{div}(u)\|_{L^{2}}^{2}\right) d s \\
\leq & c_{20} \int_{0}^{t}\left(1+\left\|\phi_{1}\right\|_{H^{1}}^{2}+\left\|\phi_{2}\right\|_{H^{1}}^{2}+\left\|U_{1}\right\|_{H^{1}}^{2}+\left\|U_{2}\right\|_{H^{1}}^{2}\right)\left(\|\varphi\|_{L^{2}}^{2}+\|u\|_{L^{2}}^{2}\right) d s \\
& +c_{21}\left(\left\|\varphi_{0}\right\|_{L^{2}}^{2}+\left\|u_{0}\right\|_{L^{2}}^{2}+\|h\|_{L^{2}(2)}^{2}\right) .
\end{aligned}
$$


Gronwall's formula implies

$$
\|\varphi\|_{\mathscr{E}}^{2}+\|u\|_{\mathbf{E}_{n}}^{2} \leq C\left(\left\|\varphi_{0}\right\|_{L^{2}}^{2}+\left\|u_{0}\right\|_{L^{2}}^{2}+\|h\|_{L^{2}(2)}^{2}\right) .
$$

By using (3.6), Green's formula, the estimate (3.12), and Lemma 1.4, we can deduce the result of the theorem.

According to Theorem 3.2, we have the following result.

Corollary 3.3. The solution of problem (3.3) is unique.

We are now going to study the differentiability of the operator solution of problem (3.3).

\section{Differentiability of the operator solution}

Before proceeding with investigation of the Fréchet differentiability of the function $\mathscr{F}$ : $\left(\varphi_{0}, u_{0}, h\right) \rightarrow(\varphi, u)$, which maps the source term $\left(\varphi_{0}, u_{0}, h\right) \in \mathscr{L}_{\infty}^{2}(\Omega) \times \mathbf{L}^{2}(\Omega) \times L^{2}(2)$ of problem (3.3) into the corresponding solution $(\varphi, u) \in \mathscr{E} \times \mathbf{E}_{n}$, we study the following problem $\left(\mathscr{P}_{I}\right)$ : find $(\psi, w) \in \mathscr{E} \times \mathbf{E}_{n}$ such that $\left(\forall(q, v) \in \mathscr{H}^{1}(\Omega) \times V_{n}\right.$ and a.e. $\left.t \in(0, T)\right)$ :

$$
\begin{aligned}
& \eta \int_{\Omega} \rho \frac{\partial \psi}{\partial t} q d x-i \eta \kappa \int_{\Omega} \operatorname{div}\left(\rho U_{1}\right) \psi q d x-i \eta \kappa \int_{\Omega} \operatorname{div}(\rho w) \phi_{1} q d x-i \mu \int_{\Gamma} \rho \psi q d \Gamma \\
& \quad+\int_{\Omega} \rho\left(B(u)(\psi)+\phi_{1} w\right) \bar{B}(u)(q) d x+\int_{\Omega} \rho B(u)\left(\phi_{1}\right) w q d x \\
& \quad+\int_{\Omega} \rho\left(\left(2\left|\phi_{1}\right|^{2}-1\right) \psi+\phi_{1}^{2} \bar{\psi}\right) q d x=0, \\
& \int_{\Omega} \rho \frac{\partial w}{\partial t} v d x+\int_{\Omega} \rho \operatorname{curl}(w) \operatorname{curl}(v) d x+\int_{\Omega} \operatorname{div}(\rho w) \operatorname{div}(v) d x+\int_{\Omega} \rho \mathscr{R}\left(\bar{\psi} B(u)\left(\phi_{1}\right)\right) v d x \\
& \quad+\int_{\Omega} \rho\left(\mathscr{R}\left(\overline{\phi_{1}} B(u)(\psi)\right)+w\left|\phi_{1}\right|^{2}\right) v d x=\int_{\Omega} \rho k \operatorname{curl}(v) d x, \\
& (\psi(0), w(0))=\left(\psi_{0}, w_{0}\right),
\end{aligned}
$$

where $U_{1}=U+u$ and $\phi_{1}=\phi+\varphi$.

Remark 4.1. The problem $\left(P_{I}\right)$ is the weak formulation of the following problem:

$$
\begin{aligned}
\eta \rho \frac{\partial \psi}{\partial t}- & i \eta \kappa\left(\operatorname{div}\left(\rho U_{1}\right) \psi+\operatorname{div}(\rho w) \phi_{1}\right)+B(u) \cdot\left(\rho\left(B(u)(\psi)+\phi_{1} w\right)\right)+\rho B(u)\left(\phi_{1}\right) w \\
& +\rho\left(\left(2\left|\phi_{1}\right|^{2}-1\right) \psi+\phi_{1}^{2} \bar{\psi}\right)=0 \quad \text { in } 2 \\
\rho \frac{\partial w}{\partial t}+ & \operatorname{curl}(\rho \operatorname{curl}(w))+\nabla(\operatorname{div}(\rho w))+\rho \mathscr{R}\left(\bar{\psi} B(u)\left(\phi_{1}\right)\right) \\
+ & \rho\left(\mathscr{R}\left(\overline{\phi_{1}} B(u)(\psi)\right)+w\left|\phi_{1}\right|^{2}\right)=\operatorname{curl}(\rho k) \quad \text { in } 2,
\end{aligned}
$$


subjected to the Robin-type boundary conditions

$$
\frac{1}{\kappa^{2}} \frac{\partial \psi}{\partial n}=\mu \psi, \quad w \cdot \mathbf{n}=0, \quad \operatorname{curl}(w)=k \quad \text { in } \Sigma,
$$

and the initial conditions

$$
(\psi(0), w(0))=\left(\psi_{0}, w_{0}\right) \quad \text { in } \Omega
$$

Theorem 4.2. If $(u, \varphi)$ and $(U, \phi)$ are in $\mathbf{W}_{n} \times\left(\mathcal{W} \cap \mathscr{L}^{\infty}(2)\right)$, then

(i) for any $\left(\psi_{0}, w_{0}, k\right) \in \mathscr{L}_{\infty}^{2}(\Omega) \times \mathbf{L}^{2}(\Omega) \times L^{2}(2)$, there exists a unique couple of functions $(\psi, w) \in \mathscr{E} \times \mathbf{E}_{n}$, solution of problem $\left(\mathscr{P}_{I}\right)$, such that

$$
\|\psi\|_{\mathscr{E}}^{2}+\|w\|_{\mathbf{E}_{n}}^{2} \leq C_{e}\left(\left\|\psi_{0}\right\|_{L^{2}}^{2}+\left\|w_{0}\right\|_{L^{2}}^{2}+\|k\|_{L^{2}(2)}^{2}\right)
$$

(ii) let $\left(\psi_{0 i}, w_{0 i}, k_{i}\right), i=1,2$, be two couples of $\in \mathscr{L}_{\infty}^{2}(\Omega) \times \mathbf{L}^{2}(\Omega) \times L^{2}(2)$. If $\left(\psi_{i}, w_{i}\right)$ is the solution of $\left(\mathscr{P}_{I}\right)$, where the initial condition is $\left(\psi_{0 i}, w_{0 i}\right), i=1,2$, then

$$
\left\|\psi_{1}-\psi_{2}\right\|_{\mathscr{E}}^{2}+\left\|w_{1}-w_{2}\right\|_{\mathbf{E}_{n}}^{2} \leq C_{e}\left(\left\|\psi_{01}-\psi_{02}\right\|_{L^{2}}^{2}+\left\|w_{01}-w_{02}\right\|_{L^{2}}^{2}+\left\|k_{1}-k_{2}\right\|_{L^{2}(2)}^{2}\right) .
$$

Proof. (i) The existence of the solution of $\left(\mathscr{P}_{I}\right)$ is obtained in the same way as to prove the Theorem 2.1 and by using the regularity of $\left(U_{1}, \phi_{1}\right)$. The uniqueness is a consequence of the estimate (4.3) (since the problem $\left(\mathscr{P}_{I}\right)$ is linear).

To prove the estimate $(4.3)$, we put $(q, v)=(\bar{\psi}, w)$ in $\left(P_{I}\right)$ and we obtain

$$
\begin{aligned}
& \eta \int_{\Omega} \rho \frac{\partial \psi}{\partial t} \bar{\psi} d x-i \eta \kappa \int_{\Omega} \operatorname{div}\left(\rho U_{1}\right)|\psi|^{2} d x-i \eta \kappa \int_{\Omega} \operatorname{div}(\rho w) \phi_{1} \bar{\psi} d x \\
& \quad-i \mu \int_{\Gamma} \rho|\psi|^{2} d \Gamma+\int_{\Omega} \rho|B(u)(\psi)|^{2} d x+\int_{\Omega} \rho\left(B(u)\left(\phi_{1}\right) \bar{\psi}+\phi_{1} \overline{B(u)(\psi)}\right) w d x \\
& \quad+\int_{\Omega} \rho\left(\left(2\left|\phi_{1}\right|^{2}-1\right)|\psi|^{2}+\phi_{1}^{2}(\bar{\psi})^{2}\right) d x=0, \\
& \int_{\Omega} \rho \frac{\partial|w|^{2}}{2 \partial t} d x+\int_{\Omega} \rho|\operatorname{curl}(w)|^{2} d x+\int_{\Omega} \operatorname{div}(\rho w) \operatorname{div}(w) d x+\int_{\Omega} \rho \mathscr{R}\left(\bar{\psi} B(u)\left(\phi_{1}\right)\right) w d x \\
& \quad+\int_{\Omega} \rho\left(\mathscr{R}\left(\overline{\phi_{1}} B(u)(\psi)\right)+w\left|\phi_{1}\right|^{2}\right) w d x=\int_{\Omega} \rho k \operatorname{curl}(w) d x, \\
& (\psi(0), w(0))=\left(\psi_{0}, w_{0}\right) .
\end{aligned}
$$

By taking the real part of the first part of previous system, by using the same technique as to prove the stability result and the regularity of $\left(U_{1}, \phi_{1}\right)$ (so we omit the details), we obtain the estimate given in (i).

(ii) Since the problem $\left(\mathscr{P}_{I}\right)$ is linear, then the result (ii) is a consequence of the estimate (4.3). 
We are now going to study the Fréchet differentiability of $\mathscr{F}$.

Theorem 4.3. (i) Let $\varphi_{0}, \varphi_{0}+\beta \in \mathscr{L}_{\infty}^{2}(\Omega)$ and $\left(u_{0}, h\right) \in \mathbf{L}^{2}(\Omega) \times L^{2}(2)$ with $\mathscr{F}\left(\varphi_{0}, u_{0}, h\right)$ and $\mathscr{F}\left(\varphi_{0}+\beta, u_{0}, h\right)$ being the corresponding solutions of (3.3). Then

$$
\left\|\mathscr{F}\left(\varphi_{0}+\beta, u_{0}, h\right)-\mathscr{F}\left(\varphi_{0}, u_{0}, h\right)-\mathscr{F}_{\varphi}^{\prime}\left(\varphi_{0}, u_{0}, h\right) \beta\right\|_{\mathscr{E} \times \mathbf{E}_{n}} \leq C\|\beta\|_{\mathscr{L}^{2}}^{3 / 2}
$$

where $\mathscr{F}_{\varphi}^{\prime}\left(\varphi_{0}, u_{0}, h\right): \mathscr{L}_{\infty}^{2}(\Omega) \rightarrow \mathscr{E} \times \mathbf{E}_{n}$ is a linear operator, and $(\psi, w)=\mathscr{F}_{\varphi}^{\prime}\left(\varphi_{0}, u_{0}, h\right) \beta$ is the solution of the problem $\left(\mathscr{P}_{I}\right)$ satisfying $(\psi, w)(t=0)=(\beta, 0)$ and $k=0$ (denote this problem by $\left.\left(\mathscr{P}_{F P}\right)\right)$.

Moreover, for all $X_{h}^{(i)}=\left(\varphi_{0 i}, u_{0 i}, h_{i}\right) \in \mathscr{L}_{\infty}^{2}(\Omega) \times \mathbf{L}^{2}(\Omega) \times L^{2}(2), i=1,2$, the following estimate exists: $\left\|\mathscr{F}_{\varphi}^{\prime}\left(X_{h}^{(1)}\right) \beta-\mathscr{F}_{\varphi}^{\prime}\left(X_{h}^{(2)}\right) \beta\right\|_{\mathscr{E} \times \mathbf{E}_{n}}^{2} \leq C_{e}\left(\|\beta\|_{\mathscr{L}^{2}}\left\|X_{h}\right\|_{\mathscr{L}^{2} \times \mathbf{L}^{2} \times L^{2}(2)}^{2}+\right.$ $\left.\|\beta\|_{\mathscr{L}^{2}}^{2}\left\|X_{h}\right\|_{\mathscr{L}^{2} \times \mathbf{L}^{2} \times L^{2}(2)}\right)$, where $\varphi_{0}=\varphi_{01}-\varphi_{02}, u_{0}=u_{01}-u_{02}, h=h_{1}-h_{2}$ and $X_{h}=X_{h}^{(1)}-$ $X_{h}^{(2)}$.

(ii) Let $u_{0}, u_{0}+z \in \mathbf{L}^{2}(\Omega)$ and $\left(\varphi_{0}, h\right) \in \mathscr{L}_{\infty}^{2}(\Omega) \times L^{2}(2)$ with $\mathscr{F}\left(\varphi_{0}, u_{0}, h\right)$ and $\mathscr{F}\left(\varphi_{0}, u_{0}\right.$ $+z, h)$ being the corresponding solutions of (3.3). Then

$$
\left\|\mathscr{F}\left(\varphi_{0}, u_{0}+z, h\right)-\mathscr{F}\left(\varphi_{0}, u_{0}, h\right)-\mathscr{F}_{u}^{\prime}\left(\varphi_{0}, u_{0}, h\right) z\right\|_{\mathscr{E} \times \mathbf{E}_{n}} \leq C\|z\|_{\mathbf{L}^{2}}^{3 / 2}
$$

where $\mathscr{F}_{u}^{\prime}\left(\varphi_{0}, u_{0}, h\right): \mathrm{L}^{2}(\Omega) \rightarrow \mathscr{E} \times \mathbf{E}_{n}$ is a linear operator, and $(\psi, w)=\mathscr{F}_{u}^{\prime}\left(\varphi_{0}, u_{0}, h\right) z$ is the solution of the problem $\left(\mathscr{P}_{I}\right)$ satisfying $(\psi, w)(t=0)=(0, z)$ and $k=0$ (denote this problem by $\left.\left(\mathscr{P}_{F u}\right)\right)$.

Moreover, for all $X_{h}^{(i)}=\left(\varphi_{0 i}, u_{0 i}, h_{i}\right) \in \mathscr{L}_{\infty}^{2}(\Omega) \times \mathbf{L}^{2}(\Omega) \times L^{2}(2), i=1,2$, the following estimate exists:

$$
\begin{aligned}
& \left\|\mathscr{F}_{u}^{\prime}\left(X_{h}^{(1)}\right) z-\mathscr{F}_{u}^{\prime}\left(X_{h}^{(2)}\right) z\right\|_{\mathscr{E} \times \mathbf{E}_{n}}^{2} \\
& \quad \leq C_{e}\left(\|z\|_{\mathbf{L}^{2}}\left\|X_{h}\right\|_{\mathscr{L}^{2} \times \mathbf{L}^{2} \times L^{2}(2)}^{2}+\|z\|_{\mathbf{L}^{2}}^{2}\left\|X_{h}\right\|_{\mathscr{L}^{2} \times \mathbf{L}^{2} \times L^{2}(2)}\right),
\end{aligned}
$$

where $\varphi_{0}=\varphi_{01}-\varphi_{02}, u_{0}=u_{01}-u_{02}, h=h_{1}-h_{2}$ and $X_{h}=X_{h}^{(1)}-X_{h}^{(2)}$.

(iii) Let $h, h+f \in L^{2}(2)$ and $\left(\varphi_{0}, u_{0}\right) \in \mathscr{L}_{\infty}^{2}(\Omega) \times \mathbf{L}^{2}(\Omega)$ with $\mathscr{F}\left(\varphi_{0}, u_{0}, h\right)$ and $\mathscr{F}\left(\varphi_{0}\right.$, $\left.u_{0}, h+f\right)$ being the corresponding solutions of (3.3). Then

$$
\left\|\mathscr{F}\left(\varphi_{0}, u_{0}, h+f\right)-\mathscr{F}\left(\varphi_{0}, u_{0}, h\right)-\mathscr{F}_{h}^{\prime}\left(\varphi_{0}, u_{0}, h\right) f\right\|_{\mathscr{E} \times \mathbf{E}_{n}} \leq C\|f\|_{L^{2}}^{3 / 2}
$$

where $\mathscr{F}_{h}^{\prime}\left(\varphi_{0}, u_{0}, h\right): L^{2}(2) \rightarrow \mathscr{E} \times \mathbf{E}_{n}$ is a linear operator, and $(\psi, w)=\mathscr{F}_{h}^{\prime}\left(\varphi_{0}, u_{0}, h\right) f$ is the solution of the problem $\left(\mathscr{P}_{I}\right)$ satisfying $(\psi, w)(t=0)=(0,0)$ and $k=f$ (denote this problem by $\left.\left(\mathscr{P}_{F h}\right)\right)$. 
Moreover, for all $X_{h}^{(i)}=\left(\varphi_{0 i}, u_{0 i}, h_{i}\right) \in \mathscr{L}_{\infty}^{2}(\Omega) \times \mathbf{L}^{2}(\Omega) \times L^{2}(2), i=1,2$, the following estimate exists:

$$
\begin{aligned}
& \left\|\mathscr{F}_{h}^{\prime}\left(X_{h}^{(1)}\right) f-\mathscr{F}_{h}^{\prime}\left(X_{h}^{(2)}\right) f\right\|_{\mathscr{E} \times \mathbf{E}_{n}}^{2} \\
& \leq C_{e}\left(\|f\|_{L^{2}(2)}\left\|X_{h}\right\|_{\mathscr{L}^{2} \times \mathbf{L}^{2} \times L^{2}(2)}^{2}+\|f\|_{L^{2}(2)}^{2}\left\|X_{h}\right\| \mathscr{L}_{\mathscr{L}^{2} \times \mathbf{L}^{2} \times L^{2}(2)}\right),
\end{aligned}
$$

where $\varphi_{0}=\varphi_{01}-\varphi_{02}, u_{0}=u_{01}-u_{02}, h=h_{1}-h_{2}$ and $X_{h}=X_{h}^{(1)}-X_{h}^{(2)}$.

Proof. According to Theorem 4.2, the problems $\left(\mathscr{P}_{F P}\right),\left(\mathscr{P}_{F u}\right)$, and $\left(\mathscr{P}_{F h}\right)$ have a unique solution in $\mathscr{E} \times \mathbf{E}_{n}$.

(i) Let be $(\varphi, u)=\mathscr{F}\left(\varphi_{0}, u_{0}, h\right)$ and $\left(\varphi_{\beta}, u_{\beta}\right)=\mathscr{F}\left(\varphi_{0}+\beta, u_{0}, h\right)$. From the stability estimate in Theorem 3.2, we know that

$$
\left\|\varphi_{\beta}-\varphi\right\|_{\mathscr{E}}^{2}+\left\|u_{\beta}-u\right\|_{\mathbf{E}_{n}}^{2} \leq C\|\beta\|_{\mathscr{L}^{2}}^{2}
$$

Denote $\phi_{\beta}=\varphi_{\beta}-\varphi, U_{\beta}=u_{\beta}-u, U_{1}=U+u, \phi_{1}=\phi+\varphi, \varphi^{*}=\phi_{\beta}-\psi, u^{*}=U_{\beta}-w$.

It is easy to see that $\left(\varphi^{*}, u^{*}\right)$ satisfies the linear problem (a.e. $\left.t \in(0, T)\right)$

$$
\begin{aligned}
& \eta \int_{\Omega} \rho \frac{\partial \varphi^{*}}{\partial t} q d x \\
&-i \eta \kappa\left(\int_{\Omega} \operatorname{div}\left(\rho U_{1}\right) \varphi^{*} q d x+\int_{\Omega} \operatorname{div}\left(\rho u^{*}\right) \phi_{1} q d x+\int_{\Omega} \operatorname{div}\left(\rho U_{\beta}\right) \phi_{\beta} q d x\right) \\
&-i \mu \int_{\Gamma} \rho \varphi^{*} q d \Gamma+\int_{\Omega} \rho\left(u^{*} \phi_{1}+B(u)\left(\varphi^{*}\right)+U_{\beta} \phi_{\beta}\right) \bar{B}(u)(q) d x \\
&+\int_{\Omega} \rho U_{\beta}\left(U_{\beta} \phi_{1}+B(u)\left(\phi_{\beta}\right)+U_{\beta} \phi_{\beta}\right) q d x \\
&+\int_{\Omega} \rho B(u)\left(\phi_{1}\right) u^{*} q d x+\int_{\Omega} \rho\left(\left(\left|\phi_{\beta}\right|^{2}+2 \mathscr{R}\left(\overline{\phi_{1}} \phi_{\beta}\right)\right) \phi_{\beta}+\phi_{1}\left|\phi_{\beta}\right|^{2}\right) q d x \\
&+\int_{\Omega} \rho\left(\left(2\left|\phi_{1}\right|^{2}-1\right) \varphi^{*}+\left(\phi_{1}\right)^{2} \overline{\varphi^{*}}\right) q d x=0, \\
& \int_{\Omega} \rho \frac{\partial u^{*}}{\partial t} v d x+\int_{\Omega} \rho \operatorname{curl}\left(u^{*}\right) \operatorname{curl}(v) d x+\int_{\Omega} \operatorname{div}\left(\rho u^{*}\right) \operatorname{div}(v) d x \\
&+\int_{\Omega} \rho \mathscr{R}\left(\overline{\phi_{\beta}} B(u)\left(\phi_{\beta}\right)\right) v d x \\
&+\int_{\Omega} \rho U_{\beta}\left(2 \mathscr{R}\left(\overline{\phi_{1}} \phi_{\beta}\right)+\left|\phi_{\beta}\right|^{2}\right) v d x+\int_{\Omega} \rho \mathscr{R}\left(\overline{\phi_{1}} B(u)\left(\varphi^{*}\right)+\overline{\varphi^{*}} B(u)\left(\phi_{1}\right)\right) v d x \\
&+\int_{\Omega} \rho u^{*}\left|\phi_{1}\right|^{2} v d x=0, \\
&\left(\varphi^{*}, u^{*}\right)(0)=(0,0) \quad \operatorname{in} \Omega .
\end{aligned}
$$


By choosing $(q, v)=\left(\overline{\varphi^{*}}, u^{*}\right)$ in $(4.12)$, we obtain (a.e. $\left.t \in(0, T)\right)$

$$
\begin{aligned}
& \eta \int_{\Omega} \rho \frac{\partial \varphi^{*}}{\partial t} \overline{\varphi^{*}} d x-i \eta \kappa\left(\int_{\Omega} \operatorname{div}\left(\rho U_{1}\right)\left|\varphi^{*}\right|^{2} d x+\int_{\Omega} \operatorname{div}\left(\rho u^{*}\right) \phi_{1} \overline{\varphi^{*}} d x\right. \\
& \left.\quad+\int_{\Omega} \operatorname{div}\left(\rho U_{\beta}\right) \phi_{\beta} \overline{\varphi^{*}} d x\right) \\
& \quad-i \mu \int_{\Gamma} \rho\left|\varphi^{*}\right|^{2} d \Gamma+\int_{\Omega} \rho\left(u^{*} \phi_{1}+B(u)\left(\varphi^{*}\right)+U_{\beta} \phi_{\beta}\right) \overline{B(u)\left(\varphi^{*}\right)} d x \\
& \quad+\int_{\Omega} \rho U_{\beta}\left(U_{\beta} \phi_{1}+B(u)\left(\phi_{\beta}\right)+U_{\beta} \phi_{\beta}\right) \overline{\varphi^{*}} d x+\int_{\Omega} \rho B(u)\left(\phi_{1}\right) u^{*} \overline{\varphi^{*}} d x \\
& \quad+\int_{\Omega} \rho\left(\left(\left|\phi_{\beta}\right|^{2}+2 \mathscr{R}\left(\overline{\phi_{1}} \phi_{\beta}\right)\right) \phi_{\beta}+\phi_{1}\left|\phi_{\beta}\right|^{2}\right) \overline{\varphi^{*}} d x \\
& \quad+\int_{\Omega} \rho\left(\left(2\left|\phi_{1}\right|^{2}-1\right)\left|\varphi^{*}\right|^{2}+\left(\phi_{1} \overline{\varphi^{*}}\right)^{2}\right) d x=0, \\
& \int_{\Omega} \rho \frac{\partial\left|u^{*}\right|^{2}}{2} d x+\int_{\Omega} \rho\left|\operatorname{curl}\left(u^{*}\right)\right|^{2} d x+\int_{\Omega} \operatorname{div}\left(\rho u^{*}\right) \operatorname{div}\left(u^{*}\right) d x+\int_{\Omega} \rho \mathscr{R}\left(\overline{\phi_{\beta}} B(u)\left(\phi_{\beta}\right)\right) u^{*} d x \\
& \quad+\int_{\Omega} \rho U_{\beta}\left(2 \mathscr{\Re}\left(\overline{\phi_{1}} \phi_{\beta}\right)+\left|\phi_{\beta}\right|^{2}\right) u^{*} d x+\int_{\Omega} \rho \mathscr{R}\left(\overline{\phi_{1}} B(u)\left(\varphi^{*}\right)+\overline{\varphi^{*}} B(u)\left(\phi_{1}\right)\right) u^{*} d x \\
& \quad+\int_{\Omega} \rho\left|u^{*}\right|^{2}\left|\phi_{1}\right|^{2} d x=0 .
\end{aligned}
$$

Since $\phi_{1}$ and $\phi_{\beta}$ are in $\mathscr{L}^{\infty}(2)$ and according to hypothesis (1.3) and Lemma 1.6, we have (by taking the real part of the first part of previous system)

$$
\begin{aligned}
\eta \rho_{0} \frac{d\left\|\varphi^{*}\right\|_{L^{2}}^{2}}{2 d t}+\frac{\rho_{0}}{\kappa^{2}}\left\|\nabla \varphi^{*}\right\|_{L^{2}}^{2} & \left(\int_{\Omega}\left|\operatorname{div}\left(\rho u^{*}\right)\right|\left|\varphi^{*}\right| d x+\int_{\Omega}\left|\operatorname{div}\left(\rho U_{\beta}\right)\right|\left|\phi_{\beta}\right|\left|\varphi^{*}\right| d x\right. \\
& +\int_{\Omega}\left(\left|u^{*}\right|+\left|U_{\beta}\right|\left|\phi_{\beta}\right|\right)\left|B(u)\left(\varphi^{*}\right)\right| d x \\
& +\int_{\Omega}\left|U_{1}\right|^{2}\left|\varphi^{*}\right|^{2} d x+\int_{\Omega}\left|U_{1}\right|\left|\varphi^{*}\right|\left|\nabla \varphi^{*}\right| d x \\
& +\int_{\Omega}\left|U_{\beta}\right|\left(\left|U_{\beta}\right|+\left|B(u)\left(\phi_{\beta}\right)\right|\right)\left|\varphi^{*}\right| d x \\
& \left.+\int_{\Omega}\left|B(u)\left(\phi_{1}\right)\right|\left|u^{*}\right|\left|\varphi^{*}\right| d x+\int_{\Omega}\left|\phi_{\beta}\right|^{2}\left|\varphi^{*}\right| d x+\int_{\Omega}\left|\varphi^{*}\right|^{2} d x\right), \\
\rho_{0} \frac{d\left\|u^{*}\right\|_{L^{2}}^{2}}{2 d t}+ & +\rho_{0}\left(\left.|| \operatorname{curl}\left(u^{*}\right)\right|_{L^{2}} ^{2}+\left.|| \operatorname{div}\left(u^{*}\right)\right|_{L^{2}} ^{2}\right) \\
\leq C( & \int_{\Omega}\left|u^{*}\right|\left|\operatorname{div}\left(u^{*}\right)\right| d x+\int_{\Omega}\left|u^{*}\right|^{2} d x+\int_{\Omega}\left|\phi_{\beta}\right|\left|B(u)\left(\phi_{\beta}\right)\right|\left|u^{*}\right| d x \\
& \left.+\int_{\Omega}\left|U_{\beta}\right|\left|\phi_{\beta}\right|\left|u^{*}\right| d x+\int_{\Omega}\left(\left|B(u)\left(\varphi^{*}\right)\right|+\left|\varphi^{*}\right|\left|B(u)\left(\phi_{1}\right)\right|\right)\left|u^{*}\right| d x\right),
\end{aligned}
$$




\section{Robust control of Ginzburg-Landau models}

and then

$$
\begin{aligned}
\eta \rho_{0} \frac{d \| \varphi^{*}}{2 d t} \|_{L^{2}}^{2} & +\frac{\rho_{0}}{\kappa^{2}}\left\|\nabla \varphi^{*}\right\|_{L^{2}}^{2} \\
\leq & C\left(\left\|U_{\beta}\right\|_{H^{1}}\left\|\phi_{\beta}\right\|_{L^{4}}\left\|\varphi^{*}\right\|_{L^{4}}+\left\|u^{*}\right\|_{H^{1}}\left\|\varphi^{*}\right\|_{L^{2}}+\left\|\varphi^{*}\right\|_{L^{2}}^{2}+\left.\left\|U_{1}\right\|\right|_{L^{4}}\left\|\varphi^{*}\right\| L_{L^{4}}\left\|\nabla \varphi^{*}\right\|_{L^{2}}\right. \\
& +\left\|U_{1}\right\|_{L^{4}}^{2}\left\|\varphi^{*}\right\|_{L^{4}}^{2} \\
& +\left(\left(1+\left\|U_{1}\right\|_{L^{4}}\right)\left(\left\|u^{*}\right\|_{L^{2}}+\left\|U_{\beta}\right\|_{L^{4}}^{2}+\left\|\phi_{\beta}\right\|_{L^{4}}^{2}\right)+\left\|U_{\beta}\right\|_{L^{4}}\left\|\phi_{\beta}\right\|_{H^{1}}\right)\left\|\varphi^{*}\right\|_{L^{4}} \\
& +\left(\left\|u^{*}\right\|_{L^{2}}+\left\|U_{\beta}\right\|_{L^{4}}\left\|\phi_{\beta}\right\|_{L^{4}}\right)\left\|\varphi^{*}\right\|_{H^{1}} \\
& \left.+\left(\left\|U_{1}\right\|_{H^{1}}+\left\|\phi_{1}\right\|_{H^{1}}\right)\left\|\varphi^{*}\right\|_{L^{4}}\left\|u^{*}\right\|_{L^{4}}+\left\|\phi_{\beta}\right\|_{L^{4}}^{4}\right), \\
\rho_{0} \frac{d\left\|u^{*}\right\|_{L^{2}}^{2}}{2 d t} & +\rho_{0}\left(\left\|\operatorname{curl}\left(u^{*}\right)\right\|_{L^{2}}^{2}+\left\|\operatorname{div}\left(u^{*}\right)\right\|_{L^{2}}^{2}\right) \\
\leq C & \left(\left\|u^{*}\right\|_{L^{2}}\left\|\operatorname{div}\left(u^{*}\right)\right\|_{L^{2}}+\left\|u^{*}\right\|_{L^{2}}^{2}+\left\|\phi_{\beta}\right\|_{L^{4}}\left(\left\|\phi_{\beta}\right\|_{H^{1}}+\left\|U_{1}\right\|_{L^{4}}\left\|\phi_{\beta}\right\|_{L^{4}}\right)\left\|u^{*}\right\|_{L^{4}}\right. \\
& +\left\|U_{\beta}\right\|_{L^{4}}\left\|\phi_{\beta}\right\|_{L^{4}}\left\|u^{*}\right\|_{L^{4}}+\left\|u^{*}\right\|_{L^{2}}\left(\left\|\varphi^{*}\right\|_{H^{1}}+\left\|U_{1}\right\|\left\|_{L^{4}}\right\| \varphi^{*} \|_{L^{4}}\right) \\
& \left.+\left\|u^{*}\right\|_{L^{4}}\left(\left\|\phi_{1}\right\|_{H^{1}}+\left\|U_{1}\right\|\left\|_{L^{4}}\right\| \phi_{1} \|_{L^{4}}\right)\left\|\varphi^{*}\right\|_{L^{4}}\right) .
\end{aligned}
$$

By using Nirenberg-Gagliardo inequality and Lemma 1.7, we have then

$$
\begin{gathered}
\eta \rho_{0} \frac{d\left\|\varphi^{*}\right\|_{L^{2}}^{2}}{2 d t}+\frac{\rho_{0}}{\kappa^{2}}\left\|\nabla \varphi^{*}\right\|_{L^{2}}^{2} \leq \frac{\rho_{0}}{4}\left(\left\|\operatorname{curl}\left(u^{*}\right)\right\|_{L^{2}}^{2}+\left\|\operatorname{div}\left(u^{*}\right)\right\|_{L^{2}}^{2}\right)+\frac{\rho_{0}}{4 \kappa^{2}}\left\|\nabla \varphi^{*}\right\|_{L^{2}}^{2} \\
+c_{1}\left(1+\left\|U_{1}\right\|_{H^{1}}^{2}+\left\|\phi_{1}\right\|_{H^{1}}^{2}+\left\|U_{1}\right\|_{L^{4}}^{4}\right)\left(\left\|u^{*}\right\|_{L^{2}}^{2}+\left\|\varphi^{*}\right\|_{L^{2}}^{2}\right) \\
+c_{2}\left(1+\left\|U_{\beta}\right\|_{H^{1}}^{2}+\left\|\phi_{\beta}\right\|_{H^{1}}^{2}\right)\left(\left\|u^{*}\right\|_{L^{2}}^{2}+\left\|\varphi^{*}\right\|_{L^{2}}^{2}\right) \\
+c_{3}\left\|U_{\beta}\right\|_{H^{1}}^{2}\left\|\phi_{\beta}\right\|_{L^{2}}+c_{4}\left\|\phi_{\beta}\right\|_{H^{1}}^{2}\left\|U_{\beta}\right\|_{L^{2}}+c_{5}\left(\left\|\phi_{\beta}\right\|_{L^{4}}^{4}+\left\|U_{\beta}\right\|_{L^{4}}^{4}\right), \\
\rho_{0} \frac{d \| u^{*}}{2 d t} \|_{L^{2}}^{2}+\rho_{0}\left(\left\|\operatorname{curl}\left(u^{*}\right)\right\|_{L^{2}}^{2}+\left\|\operatorname{div}\left(u^{*}\right)\right\|_{L^{2}}^{2}\right) \leq \frac{\rho_{0}}{4}\left(\left\|\operatorname{curl}\left(u^{*}\right)\right\|_{L^{2}}^{2}+\left\|\operatorname{div}\left(u^{*}\right)\right\|_{L^{2}}^{2}\right) \\
+\frac{\rho_{0}}{4 \kappa^{2}}\left\|\nabla \varphi^{*}\right\|_{L^{2}}^{2}+c_{6}\left(1+\left\|U_{1}\right\|_{H^{1}}^{2}+\left\|\phi_{1}\right\|_{H^{1}}^{2}+\left\|U_{1}\right\|_{L^{4}}^{4}\right)\left(\left\|u^{*}\right\|_{L^{2}}^{2}+\left\|\varphi^{*}\right\|_{L^{2}}^{2}\right) \\
+c_{7}\left\|\phi_{\beta}\right\|_{H^{1}}^{2}\left\|\phi_{\beta}\right\|_{L^{2}}+c_{8}\left(\left\|\phi_{\beta}\right\|_{L^{4}}^{4}+\left\|U_{\beta}\right\|_{L^{4}}^{4}\right) .
\end{gathered}
$$


By adding the first part and the second part of (4.16) and integrating in time, we obtain

$$
\begin{aligned}
\left\|\varphi^{*}\right\|_{L^{2}}^{2} & +\left\|u^{*}\right\|_{L^{2}}^{2}+\int_{0}^{t}\left(\left\|\nabla \varphi^{*}\right\|_{L^{2}}^{2}+\left\|\operatorname{curl}\left(u^{*}\right)\right\|_{L^{2}}^{2}+\left\|\operatorname{div}\left(u^{*}\right)\right\|_{L^{2}}^{2}\right) d s \\
\leq & c_{9} \int_{0}^{t}\left(1+\left\|U_{1}\right\|_{H^{1}}^{2}+\left\|\phi_{1}\right\|_{H^{1}}^{2}+\left\|U_{1}\right\|_{L^{4}}^{4}+\left\|U_{\beta}\right\|_{H^{1}}^{2}+\left\|\phi_{\beta}\right\|_{H^{1}}^{2}\right)\left(\left\|u^{*}\right\|_{L^{2}}^{2}+\left\|\varphi^{*}\right\|_{L^{2}}^{2}\right) d s \\
& +c_{10} \int_{0}^{t}\left(\left\|U_{\beta}\right\|_{H^{1}}^{2}+\left\|\phi_{\beta}\right\|_{H^{1}}^{2}\right)\left(\left\|\phi_{\beta}\right\|_{L^{2}}+\left\|U_{\beta}\right\|_{L^{2}}\right) d s+\int_{0}^{t}\left(\left\|\phi_{\beta}\right\|_{L^{4}}^{4}+\left\|U_{\beta}\right\|_{L^{4}}^{4}\right) d s .
\end{aligned}
$$

By using again Nirenberg-Gagliardo inequality, we have (since all functions in previous result are in $\left.L^{\infty}\left(0, T, L^{2}(\Omega)\right)\right)$

$$
\begin{aligned}
\left(\left\|\varphi^{*}\right\|_{L^{2}}^{2}+\left\|u^{*}\right\|_{L^{2}}^{2}\right)+\int_{0}^{t}\left(\left\|\nabla \varphi^{*}\right\|_{L^{2}}^{2}+\left\|\operatorname{curl}\left(u^{*}\right)\right\|_{L^{2}}^{2}+\left\|\operatorname{div}\left(u^{*}\right)\right\|_{L^{2}}^{2}\right) d s \\
\leq c_{11} \int_{0}^{t}\left(1+\left\|U_{1}\right\|_{H^{1}}^{2}+\left\|\phi_{1}\right\|_{H^{1}}^{2}+\left\|U_{\beta}\right\|_{H^{1}}^{2}+\left\|\phi_{\beta}\right\|_{H^{1}}^{2}\right)\left(\left\|u^{*}\right\|_{L^{2}}^{2}+\left\|\varphi^{*}\right\|_{L^{2}}^{2}\right) d s \\
\quad+c_{12}\left(\left\|\left(\phi_{\beta}, U_{\beta}\right)\right\|_{\mathscr{E} \times \mathbf{E}_{n}}^{3}+\left\|\left(\phi_{\beta}, U_{\beta}\right)\right\|_{\mathscr{E} \times \mathbf{E}_{n}}^{4}\right) .
\end{aligned}
$$

Using now Gronwall's formula and the stability result (4.11), we can deduce that (since all functions in previous inequality are bounded in $\left.L^{2}\left(0, T, H^{1}(\Omega)\right)\right)\left\|\left(\varphi^{*}, u^{*}\right)\right\|_{\mathscr{E} \times \mathbf{E}_{n}} \leq$ $\|\beta\|_{L^{2}}^{3 / 2}$. Therefore, we have the first part of (i).

Prove now the second part of (i).

Let $\left(\varphi_{0 i}, u_{0 i}, h_{i}\right)$ be in $\mathscr{L}_{\infty}^{2}(\Omega) \times \mathbf{L}^{2}(\Omega) \times L^{2}(2), i=1,2$, (given) and $\left(\psi_{i}, w_{i}\right)=\mathscr{F}_{\varphi}^{\prime}\left(\varphi_{0 i}\right.$, $\left.u_{0 i}, h_{i}\right) \cdot \beta$ be solution of $\left(\mathscr{P}_{F P}\right)$ (we denote by $\left(\varphi_{i}, u_{i}\right)=\mathscr{F}\left(\varphi_{0 i}, u_{0 i}, h_{i}\right)$ and by $(\varphi, u)=\left(\varphi_{1}-\right.$ $\left.\left.\varphi_{2}, u_{1}-u_{2}\right)\right)$.

Set $(\psi, w)=\left(\psi_{1}-\psi_{2}, w_{1}-w_{2}\right),\left(\varphi_{0}, u_{0}, h\right)=\left(\varphi_{01}-\varphi_{02}, u_{01}-u_{02}, h_{1}-h_{2}\right), U_{i}=u_{i}+U$ and $\phi_{i}=\varphi_{i}+\phi,(i=1,2)$. According to the equations satisfied by $\left(\psi_{1}, w_{1}\right)$ and $\left(\psi_{2}, w_{2}\right)$ we have $\left(\forall(q, v) \in \mathscr{H}^{1}(\Omega) \times V_{n}\right.$ and a.e. $\left.t \in(0, T)\right)$ :

$$
\begin{aligned}
\eta \int_{\Omega} \rho \frac{\partial \psi}{\partial t} & q d x-i \eta \kappa \int_{\Omega}\left(\operatorname{div}\left(\rho U_{2}\right) \psi+\operatorname{div}(\rho w) \phi_{1}\right) q d x \\
& -i \eta \kappa \int_{\Omega}\left(\operatorname{div}(\rho u) \psi_{1}+\operatorname{div}\left(\rho w_{2}\right) \varphi\right) q d x \\
& -i \mu \int_{\Gamma} \rho \psi q d \Gamma+\int_{\Omega} \rho\left(B\left(u_{2}\right)(\psi)+u \psi_{1}+\varphi w_{1}+\phi_{2} w\right) \bar{B}\left(u_{2}\right)(q) d x \\
& +\int_{\Omega} \rho\left(B\left(u_{1}\right)\left(\psi_{1}\right)+w_{1} \phi_{1}\right) u q d x+\int_{\Omega} \rho B\left(u_{1}\right)\left(\phi_{1}\right) w q d x \\
& +\int_{\Omega} \rho\left(B\left(u_{2}\right)(\varphi)+u \phi_{1}\right) w_{2} q d x \\
& +\int_{\Omega} \rho\left(\left(2\left|\phi_{1}\right|^{2}-1\right) \psi+\phi_{1}^{2} \bar{\psi}\right) q d x \\
& +\int_{\Omega} \rho\left(2\left(|\varphi|^{2}+2 \mathscr{R}\left(\varphi \overline{\phi_{2}}\right)\right) \psi_{2}+\varphi\left(\phi_{1}+\phi_{2}\right) \overline{\psi_{2}}\right) q d x=0
\end{aligned}
$$




$$
\begin{aligned}
& \int_{\Omega} \rho \frac{\partial w}{\partial t} v d x+\int_{\Omega} \rho \operatorname{curl}(w) \operatorname{curl}(v) d x+\int_{\Omega} \operatorname{div}(\rho w) \operatorname{div}(v) d x \\
&+\int_{\Omega} \rho\left(\mathscr{R}\left(\bar{\psi} B\left(u_{1}\right)\left(\phi_{1}\right)\right)+w\left|\phi_{1}\right|^{2}\right) v d x \\
&+\int_{\Omega} \rho \mathscr{R}\left(\overline{\phi_{2}}\left(B\left(u_{2}\right)(\psi)+u \psi_{1}\right)+\varphi B\left(u_{1}\right)\left(\psi_{1}\right)\right) v d x \\
&+\int_{\Omega} \rho \mathscr{R}\left(\overline{\psi_{2}}\left(B\left(u_{2}\right)(\varphi)+u \phi_{1}\right)\right) v d x+\int_{\Omega} \rho w_{2}\left(|\varphi|^{2}+2 \mathscr{R}\left(\varphi \phi_{2}\right)\right) v d x=0, \\
&(\psi(0), w(0))=(0,0) .
\end{aligned}
$$

Putting $(q, v)=(\bar{\psi}, w)$ in $(4.19)$, we have then

$$
\begin{aligned}
& \eta \int_{\Omega} \rho \frac{\partial \psi}{\partial t} \bar{\psi} d x-i \eta \kappa \int_{\Omega}\left(\operatorname{div}\left(\rho U_{2}\right)|\psi|^{2}+\operatorname{div}(\rho w) \phi_{1} \bar{\psi}\right) d x \\
& -i \eta \kappa \int_{\Omega}\left(\operatorname{div}(\rho u) \psi_{1}+\operatorname{div}\left(\rho w_{2}\right) \varphi\right) \bar{\psi} d x \\
& -i \mu \int_{\Gamma} \rho|\psi|^{2} d \Gamma+\int_{\Omega} \rho\left(B\left(u_{2}\right)(\psi)+u \psi_{1}+\varphi w_{1}+\phi_{2} w\right) \overline{B\left(u_{2}\right)(\psi)} d x \\
& +\int_{\Omega} \rho\left(B\left(u_{1}\right)\left(\psi_{1}\right)+w_{1} \phi_{1}\right) u \bar{\psi} d x+\int_{\Omega} \rho B\left(u_{1}\right)\left(\phi_{1}\right) w \bar{\psi} d x \\
& +\int_{\Omega} \rho\left(B\left(u_{2}\right)(\varphi)+u \phi_{1}\right) w_{2} \bar{\psi} d x \\
& +\int_{\Omega} \rho\left(\left(2\left|\phi_{1}\right|^{2}-1\right) \psi+\phi_{1}^{2} \bar{\psi}\right) \bar{\psi} d x \\
& +\int_{\Omega} \rho\left(2\left(|\varphi|^{2}+2 \Re\left(\varphi \overline{\phi_{2}}\right)\right) \psi_{2}+\varphi\left(\phi_{1}+\phi_{2}\right) \overline{\psi_{2}}\right) \bar{\psi} d x=0, \\
& \int_{\Omega} \rho \frac{\partial|w|^{2}}{2 \partial t} d x+\int_{\Omega} \rho|\operatorname{curl}(w)|^{2} d x+\int_{\Omega} \operatorname{div}(\rho w) \operatorname{div}(w) d x \\
& +\int_{\Omega} \rho\left(\mathscr{R}\left(\bar{\psi} B\left(u_{1}\right)\left(\phi_{1}\right)\right)+w\left|\phi_{1}\right|^{2}\right) w d x \\
& +\int_{\Omega} \rho \mathscr{R}\left(\overline{\phi_{2}}\left(B\left(u_{2}\right)(\psi)+u \psi_{1}\right)+\varphi B\left(u_{1}\right)\left(\psi_{1}\right)\right) w d x \\
& +\int_{\Omega} \rho \mathscr{R}\left(\overline{\psi_{2}}\left(B\left(u_{2}\right)(\varphi)+u \phi_{1}\right)\right) w d x+\int_{\Omega} \rho w_{2}\left(|\varphi|^{2}+2 \mathscr{R}\left(\varphi \phi_{2}\right)\right) w d x=0 \text {, } \\
& (\psi(0), w(0))=(0,0) \text {. }
\end{aligned}
$$

By taking the real part of the first part of previous system, we obtain ( since $\left(\phi_{i}\right)_{i=1,2}, \varphi$ are 
in $\mathscr{L}^{\infty}(2)$ and according to Lemma 1.6)

$$
\begin{aligned}
& \eta \rho_{0} \frac{d\|\psi\|_{L^{2}}^{2}}{2 d t}+\frac{\rho_{0}}{\kappa^{2}}\|\nabla \psi\|_{L^{2}}^{2} \\
& \leq C\left(\|w\|_{H^{1}}\|\psi\|_{L^{2}}+\|u\|_{H^{1}}\left\|\psi_{1}\right\|_{L^{4}}\|\psi\|_{L^{4}}+\left\|w_{2}\right\|_{H^{1}}\|\varphi\|_{L^{4}}\|\psi\|_{L^{4}}\right. \\
& +\left(\|\psi\|_{H^{1}}+\left\|U_{2}\right\|_{L^{4}}\|\psi\|_{L^{4}}\right)\left(\|u\|_{L^{4}}\left\|\psi_{1}\right\|_{L^{4}}+\left\|w_{1}\right\|_{L^{4}}\|\varphi\|_{L^{4}}+\|w\|_{L^{2}}+\left\|U_{2}\right\|_{L^{4}}\|\psi\|_{L^{4}}\right) \\
& +\|u\|_{L^{4}}\|\psi\|_{L^{4}}\left(\left\|w_{1}\right\|_{L^{2}}+\left\|\psi_{1}\right\|_{H^{1}}+\left\|U_{1}\right\|_{L^{4}}\left\|\psi_{1}\right\|_{L^{4}}\right) \\
& +\|\psi\|_{L^{4}}\|w\|_{L^{4}}\left(\left\|\phi_{1}\right\|_{H^{1}}+\left\|U_{1}\right\|_{L^{2}}\right) \\
& +\left\|w_{2}\right\|_{L^{4}}\|\psi\|_{L^{4}}\left(\|\varphi\|_{H^{1}}+\left\|U_{2}\right\|_{L^{4}}\|\varphi\|_{L^{4}}+\|u\|_{L^{2}}\right)+\|\psi\|_{L^{2}}^{2} \\
& \left.+\left\|\psi_{2}\right\|_{L^{4}}\|\varphi\|_{L^{4}}\|\psi\|_{L^{4}}\right) \\
& \rho_{0} \frac{d\|w\|_{L^{2}}^{2}}{2 d t}+\rho_{0}\left(\|\operatorname{curl}(w)\|_{L^{2}}^{2}+\|\operatorname{div}(w)\|_{L^{2}}^{2}\right) \\
& \leq \frac{\rho_{0}}{4}\left(\|\operatorname{curl}(w)\|_{L^{2}}^{2}+\|\operatorname{div}(w)\|_{L^{2}}^{2}\right) \\
& +C\|w\|_{L^{4}}\|\psi\|_{L^{4}}\left(\left\|\phi_{1}\right\|_{H^{1}}+\left\|U_{1}\right\|_{L^{2}}\right) \\
& +\|w\|_{L^{2}}\left(1+\|\psi\|_{H^{1}}+\left\|U_{2}\right\|_{L^{4}}\|\psi\|_{L^{4}}+\|u\|_{L^{4}}\left\|\psi_{1}\right\|_{L^{4}}\right) \\
& +\|w\|_{L^{4}}\|\varphi\|_{L^{4}}\left(\left\|\psi_{1}\right\|_{H^{1}}+\left\|U_{1}\right\|_{L^{4}}\left\|\psi_{1}\right\|_{L^{4}}+\left\|w_{2}\right\|_{L^{4}}\right) \\
& +\|w\|_{L^{4}}\left\|\psi_{2}\right\|_{L^{4}}\left(\left\|U_{2}\right\|_{L^{4}}\|\varphi\|_{L^{4}}+\|\varphi\|_{H^{1}}+\|u\|_{L^{2}}\right) .
\end{aligned}
$$

By using Lemma 1.7 and Young's formula, we can deduce that

$$
\begin{aligned}
\eta \rho_{0} \frac{d\|\psi\|_{L^{2}}^{2}}{2 d t}+\frac{\rho_{0}}{\kappa^{2}}\|\nabla \psi\|_{L^{2}}^{2} \\
\leq \frac{\rho_{0}}{4 \kappa^{2}}\|\nabla \psi\|_{L^{2}}^{2}+\frac{\rho_{0}}{4}\left(\|\operatorname{curl}(w)\|_{L^{2}}^{2}+\|\operatorname{div}(w)\|_{L^{2}}^{2}\right) \\
\quad+c_{0}\|\psi\|_{L^{2}}\left(1+\left\|U_{2}\right\|_{H^{1}}^{2}+\left\|U_{2}\right\|_{L^{4}}^{4}\right) \\
\quad+c_{1}\|\psi\|_{L^{2}}\left(\left\|\left(\phi_{1}, U_{1}\right)\right\|_{H^{1}}^{2}+\|(\varphi, u)\|_{H^{1}}^{2}+\left\|\left(\psi_{1}, w_{1}\right)\right\|_{H^{1}}^{2}+\left\|\left(\psi_{2}, w_{2}\right)\right\|_{H^{1}}^{2}\right) \\
\quad+c_{2}\left(1+\left\|\left(\phi_{1}, U_{1}\right)\right\|_{H^{1}}^{2}\right)\|w\|_{L^{2}}^{2}+c_{3}\|(\varphi, u)\|_{L^{4}}^{2}\left(\left\|\left(\psi_{1}, w_{1}\right)\right\|_{L^{4}}^{2}+\left\|\left(\psi_{2}, w_{2}\right)\right\|_{L^{4}}^{2}\right) \\
\quad+c_{4}\left(\|(\varphi, u)\|_{L^{2}}\left(\left\|\left(\psi_{1}, w_{1}\right)\right\|_{H^{1}}^{2}+\left\|\left(\psi_{2}, w_{2}\right)\right\|_{H^{1}}^{2}\right)\right. \\
\left.\quad+\|(\varphi, u)\|_{H^{1}}^{2}\left(\left\|\left(\psi_{1}, w_{1}\right)\right\|_{L^{2}}+\left\|\left(\psi_{2}, w_{2}\right)\right\|_{L^{2}}\right)\right)
\end{aligned}
$$


Robust control of Ginzburg-Landau models

$$
\begin{aligned}
& \rho_{0} \frac{d\|w\|_{L^{2}}^{2}}{2 d t}+\rho_{0}\left(\|\operatorname{curl}(w)\|_{L^{2}}^{2}+\|\operatorname{div}(w)\|_{L^{2}}^{2}\right) \\
& \leq \frac{\rho_{0}}{4}\left(\|\operatorname{curl}(w)\|_{L^{2}}^{2}+\|\operatorname{div}(w)\|_{L^{2}}^{2}\right)+\frac{\rho_{0}}{4 \kappa^{2}}\|\nabla \psi\|_{L^{2}}^{2}+c_{5}\|w\|_{L^{2}}\left(1+\left\|U_{2}\right\|_{H^{1}}^{2}+\left\|U_{2}\right\|_{L^{4}}^{4}\right) \\
& \quad+c_{6}\|w\|_{L^{2}}\left(\left\|\left(\phi_{1}, U_{1}\right)\right\|_{H^{1}}^{2}+\|(\varphi, u)\|_{H^{1}}^{2}+\left\|\left(\psi_{1}, w_{1}\right)\right\|_{H^{1}}^{2}+\left\|\left(\psi_{2}, w_{2}\right)\right\|_{H^{1}}^{2}\right) \\
& +c_{7}\left(1+\left\|\left(\phi_{1}, U_{1}\right)\right\|_{H^{1}}^{2}+\left\|U_{2}\right\|_{L^{4}}^{4}\right)\|\psi\|_{L^{2}}^{2} \\
& +c_{8}\|(\varphi, u)\|_{L^{4}}^{2}\left(\left\|\left(\psi_{1}, w_{1}\right)\right\|_{L^{4}}^{2}+\left\|\left(\psi_{2}, w_{2}\right)\right\|_{L^{4}}^{2}\right) \\
& +c_{9}\left(\|(\varphi, u)\|_{L^{2}}\left(\left\|\left(\psi_{1}, w_{1}\right)\right\|_{H^{1}}^{2}+\left\|\left(\psi_{2}, w_{2}\right)\right\|_{H^{1}}^{2}\right)\right. \\
& \left.\quad+\|(\varphi, u)\|_{H^{1}}^{2}\left(\left\|\left(\psi_{1}, w_{1}\right)\right\|_{L^{2}}+\left\|\left(\psi_{2}, w_{2}\right)\right\|_{L^{2}}\right)\right) .
\end{aligned}
$$

Integrating over $(0, t)$ for $t \in(0, T)$ and using Theorems 3.2 and 4.2, we obtain then (since $\left(\phi_{i}, U_{i}\right),\left(\psi_{i}, w_{i}\right), i=1,2$, and $(\varphi, u)$ are in $\left.L^{\infty}\left(0, T, L^{2}(\Omega)\right)\right)$

$$
\begin{aligned}
\eta \rho_{0}\|\psi\|_{L^{2}}^{2}+\frac{2 \rho_{0}}{\kappa^{2}} \int_{0}^{t}\|\nabla \psi\|_{L^{2}}^{2} d s \\
\leq \frac{\rho_{0}}{2 \kappa^{2}} \int_{0}^{t}\|\nabla \psi\|_{L^{2}}^{2} d s+\frac{\rho_{0}}{2} \int_{0}^{t}\left(\|\operatorname{curl}(w)\|_{L^{2}}^{2}+\|\operatorname{div}(w)\|_{L^{2}}^{2}\right) d s \\
\quad+c_{10} \int_{0}^{t}\|\psi\|_{L^{2}}\left(1+\left\|U_{2}\right\|_{H^{1}}^{2}\right) d s \\
\quad+c_{11} \int_{0}^{t}\|\psi\|_{L^{2}}\left(\left\|\left(\phi_{1}, U_{1}\right)\right\|_{H^{1}}^{2}+\|(\varphi, u)\|_{H^{1}}^{2}+\left\|\left(\psi_{1}, w_{1}\right)\right\|_{H^{1}}^{2}+\left\|\left(\psi_{2}, w_{2}\right)\right\|_{H^{1}}^{2}\right) d s \\
\quad+c_{12} \int_{0}^{t}\left(1+\left\|\left(\phi_{1}, U_{1}\right)\right\|_{H^{1}}^{2}\right)\|w\|_{L^{2}}^{2} d s+c_{13}\left(\|X\|_{L^{2}}^{2}\|\beta\|_{L^{2}}+\|\beta\|_{L^{2}}^{2}\left\|X_{h}\right\|_{L^{2}}\right),
\end{aligned}
$$

$\rho_{0}\|w\|_{L^{2}}^{2}+2 \rho_{0} \int_{0}^{t}\left(\|\operatorname{curl}(w)\|_{L^{2}}^{2}+\|\operatorname{div}(w)\|_{L^{2}}^{2}\right) d s$

$$
\begin{aligned}
\leq & \frac{\rho_{0}}{2} \int_{0}^{t}\left(\|\operatorname{curl}(w)\|_{L^{2}}^{2}+\|\operatorname{div}(w)\|_{L^{2}}^{2}\right) d s+\frac{\rho_{0}}{2 \kappa^{2}} \int_{0}^{t}\|\nabla \psi\|_{L^{2}}^{2} d s \\
& +c_{14} \int_{0}^{t}\|w\|_{L^{2}}\left(1+\left\|U_{2}\right\|_{H^{1}}^{2}\right) d s \\
& +c_{15} \int_{0}^{t}\|w\|_{L^{2}}\left(\left\|\left(\phi_{1}, U_{1}\right)\right\|_{H^{1}}^{2}+\|(\varphi, u)\|_{H^{1}}^{2}+\left\|\left(\psi_{1}, w_{1}\right)\right\|_{H^{1}}^{2}+\left\|\left(\psi_{2}, w_{2}\right)\right\|_{H^{1}}^{2}\right) d s \\
& +c_{16} \int_{0}^{t}\left(1+\left\|\left(\phi_{1}, U_{1}\right)\right\|_{H^{1}}^{2}+\left\|U_{2}\right\|_{L^{4}}^{4}\right)\|\psi\|_{L^{2}}^{2} d s+c_{17}\left(\|X\|_{L^{2}}^{2}\|\beta\|_{L^{2}}+\|\beta\|_{L^{2}}^{2}\left\|X_{h}\right\|_{L^{2}}\right),
\end{aligned}
$$

where $X_{h}=\left(\varphi_{0}, u_{0}, h\right)$. 
The previous statements lead to

$$
\begin{aligned}
\|\psi\|_{L^{2}}^{2}+ & \|w\|_{L^{2}}^{2}+\int_{0}^{t}\left(\|\nabla \psi\|_{L^{2}}^{2}+\|\operatorname{curl}(w)\|_{L^{2}}^{2}+\|\operatorname{div}(w)\|_{L^{2}}^{2}\right) d s \\
\leq & c_{18} \int_{0}^{t}\left(\|\psi\|_{L^{2}}^{2}+\|w\|_{L^{2}}^{2}\right)\left(1+\left\|\left(\phi_{1}, U_{1}\right)\right\|_{H^{1}}^{2}+\left\|U_{2}\right\|_{H^{1}}^{2}\right) d s \\
& +c_{19} \int_{0}^{t}\left(\|\psi\|_{L^{2}}^{2}+\|w\|_{L^{2}}^{2}\right)\left(\|(\varphi, u)\|_{H^{1}}^{2}+\left\|\left(\psi_{1}, w_{1}\right)\right\|_{H^{1}}^{2}+\left\|\left(\psi_{2}, w_{2}\right)\right\|_{H^{1}}^{2}\right) d s \\
& +c_{20}\left(\|X\|_{L^{2}}^{2}\|\beta\|_{L^{2}}+\|\beta\|_{L^{2}}^{2}\left\|X_{h}\right\|_{L^{2}}\right) .
\end{aligned}
$$

According to Gronwall's formula, we can deduce the second part of (i) (since $\left(\phi_{i}, U_{i}\right)_{i=1,2}$, $\left(\psi_{i}, w_{i}\right)_{i=1,2}$ and $(\varphi, u)$ are in $\left.L^{2}\left(0, T, H^{1}(\Omega)\right)\right)$.

By using the same technique to prove the results of (i), we have the result of (ii) and (iii). So, we omit the tedious details.

\section{Robust control problem}

The objective of the robust control problem is to find the best admissible control in the presence of the worst disturbance which maximally spoils the control objective. We formulated the problem in two situations: firstly the case where the control is in the external magnetic field and secondly the case where the control is in the initial condition $u_{0}$ (data assimilation).

5.1. The control in the external magnetic field. In this section, we consider two situations: firstly the case where the worst disturbance is in the external magnetic field $h$ and secondly the case where the disturbance is in the initial condition $\varphi_{0}$.

5.1.1. Distributed disturbance in the external magnetic field. We suppose now that the value $h$ is decomposed into the disturbance $f \in L^{2}(2)$ and the control $g \in L^{2}(2)$, that is, $h=f+g$. So the function $(\varphi, u)$ is assumed to be related to the disturbance $f$ and control $g$ through the problem $(3.3)\left(\forall(q, v) \in \mathscr{H}^{1}(\Omega) \times V_{n}\right.$ and a.e. $\left.t \in(0, T)\right)$

$$
\begin{aligned}
& \eta \int_{\Omega} \rho \frac{\partial \varphi}{\partial t} q d x-i \eta \kappa \int_{\Omega} \operatorname{div}(\rho(u+U)) \varphi q d x-i \eta \kappa \int_{\Omega} \operatorname{div}(\rho u) \phi q d x-i \mu \int_{\Gamma} \rho \varphi q d \Gamma \\
& \quad+\int_{\Omega} \rho B(u)(\varphi+\phi) \bar{B}(u)(q) d x+\int_{\Omega} \rho F(\varphi) q d x=\int_{\Omega} \rho B(0)(\phi) \bar{B}(0)(q) d x \\
& \int_{\Omega} \rho \frac{\partial u}{\partial t} v d x+\int_{\Omega} \rho \operatorname{curl}(u) \operatorname{curl}(v) d x+\int_{\Omega} \operatorname{div}(\rho u) \operatorname{div}(v) d x \\
& \quad+\int_{\Omega} \rho \mathscr{R}((\bar{\varphi}+\bar{\phi}) B(u)(\varphi+\phi)) v d x \\
& =\int_{\Omega} \rho \mathscr{R}(\bar{\phi} B(0)(\phi)) v d x+\int_{\Omega} \rho(f+g) \operatorname{curl}(v) d x \\
& (\varphi(0), u(0))=\left(\varphi_{0}, u_{0}\right) .
\end{aligned}
$$


To obtain the regularity of Theorem 4.2, we suppose the following hypotheses: $\left(\varphi_{0}, u_{0}\right) \in$ $\mathscr{L}_{\infty}^{2}(\Omega) \times \mathbf{L}^{2}(\Omega)$. Let $\mathscr{P}:(f, g) \rightarrow(\varphi, u)=\mathscr{P}(f, g)$ be the map: $\left(L^{2}(2)\right)^{2} \rightarrow \mathscr{E} \times \mathbf{E}_{n}$ defined by (5.1) and introduce the cost function defined by

$$
J(f, g)=\frac{a}{4}\left\||\varphi|^{2}-\Lambda\right\|_{L^{2}(2)}^{2}+\frac{b}{2}\left\|u-u_{\mathrm{obs}}\right\|_{L^{2}(2)}^{2}+\frac{\alpha}{2}\|f\|_{L^{2}(2)}^{2}-\frac{\gamma}{2}\|g\|_{L^{2}(2)}^{2}
$$

where $a, b, \alpha, \gamma$ are fixed such that $\alpha, \gamma>0, a, b \geq 0$, and $a+b>0$. The functions $u_{\mathrm{obs}} \in$ $\mathbf{L}^{2}(2)$ and $\Lambda \in L^{\infty}(2)$ are given and represent the observation.

Let $\mathscr{K}=\mathscr{K}_{1} \times \mathscr{K}_{2}$ such that $\mathscr{K}_{1}$ and $\mathscr{K}_{2}$ are (given) nonempty, closed, convex, bounded subsets of $L^{2}(2)$. We want to minimize the functional $J$ with respect to $f$ and maximize $J$ with respect to $g$, that is, to study the following problem $\left(M \mathscr{P}_{1}\right)$ :

find an admissible control $f^{*} \in \mathscr{K}_{1}$ and a disturbance $g^{*} \in \mathscr{K}_{2}$ such that $\left(f^{*}, g^{*}\right)$ is a saddle point of the functional $J$ on $\mathcal{K}$, subject to system (5.1).

Such a pair $\left(f^{*}, g^{*}\right)$ is called an optimal solution to $\left(\mathcal{M} \mathscr{P}_{1}\right)$.

Proposition 5.1. The function $\mathscr{P}$ is continuously Fréchet differentiable from $\left(L^{2}(2)\right)^{2}$ to $\mathscr{E} \times \mathbf{E}_{n}$ with the derivative $\mathscr{P}^{\prime}(f, g): Y=\left(\beta_{1}, \beta_{2}\right) \rightarrow(\psi, w)$ given by the linear problem $\left(\mathscr{P}_{F 1}\right)\left(\forall(q, v) \in \mathscr{H}^{1}(\Omega) \times V_{n}\right.$ and a.e. $\left.t \in(0, T)\right)$

$$
\begin{aligned}
& \eta \int_{\Omega} \rho \frac{\partial \psi}{\partial t} q d x-i \eta \kappa \int_{\Omega} \operatorname{div}\left(\rho U_{1}\right) \psi q d x-i \eta \kappa \int_{\Omega} \operatorname{div}(\rho w) \phi_{1} q d x-i \mu \int_{\Gamma} \rho \psi q d \Gamma \\
& \quad+\int_{\Omega} \rho\left(B(u)(\psi)+\phi_{1} w\right) \bar{B}(u)(q) d x+\int_{\Omega} \rho B(u)\left(\phi_{1}\right) w q d x \\
& \quad+\int_{\Omega} \rho\left(\left(2\left|\phi_{1}\right|^{2}-1\right) \psi+\phi_{1}^{2} \bar{\psi}\right) q d x=0, \\
& \int_{\Omega} \rho \frac{\partial w}{\partial t} v d x+\int_{\Omega} \rho \operatorname{curl}(w) \operatorname{curl}(v) d x+\int_{\Omega} \operatorname{div}(\rho w) \operatorname{div}(v) d x+\int_{\Omega} \rho \mathscr{R}\left(\bar{\psi} B(u)\left(\phi_{1}\right)\right) v d x \\
& \quad+\int_{\Omega} \rho\left(\mathscr{R}\left(\overline{\phi_{1}} B(u)(\psi)\right)+w\left|\phi_{1}\right|^{2}\right) v d x=\int_{\Omega} \rho\left(\beta_{1}+\beta_{2}\right) \operatorname{curl}(v) d x \\
& (\psi(0), w(0))=(0,0),
\end{aligned}
$$

where $\left(U_{1}, \phi_{1}\right)=(u+U, \varphi+\phi)$.

Moreover the following estimates exist: $\left(\forall\left(f_{i}, g_{i}\right) \in\left(L^{2}(\Omega)\right)^{2}, i=1,2\right)$

(i) $\left\|\mathscr{P}^{\prime}\left(f_{1}, g_{1}\right)\right\| \mathscr{L}\left(\left(L^{2}(2)\right)^{2}, \mathscr{E} \times \mathbf{E}_{n}\right) \leq C_{e}$;

(ii) $\left\|\mathscr{P}^{\prime}\left(f_{1}, g_{1}\right) Y-\mathscr{P}^{\prime}\left(f_{2}, g_{2}\right) Y\right\|_{\mathscr{E} \times \mathbf{E}_{n}}^{2} \leq C_{e}\left(\|X\|_{L^{2}(2)}\|Y\|_{L^{2}(2)}^{2}+\|X\|_{L^{2}(2)}^{2}\|Y\|_{L^{2}(2)}\right)$, where $f=f_{1}-f_{2}, g=g_{1}-g_{2}$ and $X=(f, g)$. 
Proof. The proof of this proposition is the consequence of the result of Theorem 4.3. Here we omit the tedious details.

Proposition 5.2. The map $\mathscr{P}$ defined by (5.1) is continuous from the weak topology of $\left(L^{2}(2)\right)^{2}$ to the strong topology of $\mathscr{L}^{2}(2) \times \mathbf{L}^{2}(2)$.

Proof. Let $\mathbf{f}=(f, g)$ be given in $\left(L^{2}(2)\right)^{2}$ and let be a sequence $\mathbf{f}_{k}=\left(f_{k}, g_{k}\right)$ such that $\mathbf{f}_{k}$ is weakly convergent in $\left(L^{2}(2)\right)^{2}$ to $\mathbf{f}$.

Set $(\varphi, u)=\mathscr{P}(f, g)$ and $\left(\varphi_{k}, u_{k}\right)=\mathscr{P}\left(f_{k}, g_{k}\right)$. Since $\mathbf{f}_{k} \rightarrow \mathbf{f}$ weakly in $\left(L^{2}(2)\right)^{2}$ then $\mathbf{f}_{k}$ is uniformly bounded in $\left(L^{2}(2)\right)^{2}$. In view of Theorem 3.2, we can deduce that the sequence $\left(\varphi_{k}, u_{k}\right)$ is uniformly bounded in $\mathscr{E} \times \mathbf{E}_{n}$. Therefore we can extract from $\left(\mathbf{f}_{k}, \varphi_{k}, u_{k}\right)$ a subsequence also denoted by $\left(\mathbf{f}_{k}, \varphi_{k}, u_{k}\right)$ and such that

$$
\begin{gathered}
\left(f_{k}, g_{k}\right) \longrightarrow(f, g) \text { weakly in }\left(L^{2}(2)\right)^{2}, \\
\left(\varphi_{k}, u_{k}\right) \longrightarrow(\tilde{\varphi}, \tilde{u}) \text { weakly in } \mathscr{E} \times \mathbf{E}_{n}, \\
\left(\varphi_{k}, u_{k}\right) \longrightarrow(\tilde{\varphi}, \tilde{u}) \text { strongly in } \mathscr{L}^{2}(\mathscr{2}) \times \mathbf{L}^{2}(\mathscr{2}), \\
\varphi_{k} \longrightarrow \tilde{\varphi} \text { weakly in } \mathscr{L}^{2}(\Sigma) .
\end{gathered}
$$

We prove easily that $(\tilde{\varphi}, \tilde{u})=\mathscr{P}(f, g)$ and according to the uniqueness of the solution of (5.1), we have then $\tilde{\varphi}=\varphi$ and $\tilde{u}=u$.

Theorem 5.3. For $\alpha$ and $\gamma$ sufficiently large (i.e., there exists $\left(\alpha_{l}, \gamma_{l}\right)$ such that $\alpha \geq \alpha_{l}$ and $\left.\gamma \geq \gamma_{l}\right)$, there exists $\left(f^{*}, g^{*}\right) \in \mathscr{Y}$ and $\left(\varphi^{*}, u^{*}\right) \in \mathscr{E} \times \mathbf{E}_{n}$ such that $\left(f^{*}, g^{*}\right)$ is defined by $\left(M \mathscr{P}_{1}\right)$ and $\left(\varphi^{*}, u^{*}\right)=\mathscr{P}\left(f^{*}, g^{*}\right)$ is solution of $(5.1)$.

Proof. Let $P_{g}$ be the map $f \rightarrow J(f, g)$ and let $Q_{f}$ be the map: $g \rightarrow J(f, g)$. To obtain the existence of the robust control problem, we prove that $P_{g}$ is convex and lower semicontinuous for all $g \in \mathscr{K}_{2}$, and $Q_{f}$ is concave and upper semicontinuous for all $f \in \mathcal{K}_{1}$ and we use the classical minimax theorem in infinite dimensions (see, e.g., $[3,23]$ ).

Firstly we prove, for $\alpha$ and $\gamma$ sufficiently large, the convexity of the map $P_{g}$ and the concavity of the map $Q_{f}$. In order to prove the convexity, it is sufficient to show that for all $\left(f_{1}, f_{2}\right) \in \mathcal{K}_{1}$ we have $\left(P_{g}^{\prime}\left(f_{1}\right)-P_{g}^{\prime}\left(f_{2}\right)\right) \cdot f \geq 0$, where $f=f_{1}-f_{2}$ (because $P_{g}$ is Gâteaux differentiable). According to the definition of $J$, we have that

$$
\begin{aligned}
\left(P_{g}^{\prime}\left(f_{1}\right)\right. & \left.-P_{g}^{\prime}\left(f_{2}\right)\right) \cdot f \\
= & \alpha\|f\|_{L^{2}(2)}^{2}+a \iint_{2} \mathscr{R}\left(\left(\left|\varphi_{1}\right|^{2}-\left|\varphi_{2}\right|^{2}\right) \psi_{2} \bar{\varphi}_{2}\right) d x d t \\
& +a \iint_{2} \mathscr{R}\left(\left(\left|\varphi_{1}\right|^{2}-\Lambda\right)\left(\varphi_{1}-\varphi_{2}\right) \bar{\psi}_{1}\right) d x d t+b \iint_{2}\left(u_{1}-u_{2}\right) w_{2} d x d t \\
& +a \iint_{2} \mathscr{R}\left(\left(\left|\varphi_{1}\right|^{2}-\Lambda\right)\left(\psi_{1}-\psi_{2}\right) \bar{\varphi}_{2}\right) d x d t+b \iint_{2}\left(u_{1}-u_{\mathrm{obs}}\right)\left(w_{1}-w_{2}\right) d x d t
\end{aligned}
$$

where $\left(\varphi_{i}, u_{i}\right)=\mathscr{P}\left(f_{i}, g\right),\left(\psi_{i}, w_{i}\right)=\mathscr{P}^{\prime}\left(f_{i}, g\right) \cdot(f, 0)$ (solution of problem $\left.\left(\mathscr{P}_{F 1}\right)\right)$, for $i=$ 1,2 . 
According to Theorem 3.2 and Proposition 5.1, we have

$$
\begin{array}{rl}
a \iint_{2} & \left(\mathscr{R}\left(\left(\left|\varphi_{1}\right|^{2}-\left|\varphi_{2}\right|^{2}\right) \psi_{2} \bar{\varphi}_{2}\right)+\mathscr{R}\left(\left(\left|\varphi_{1}\right|^{2}-\Lambda\right)\left(\varphi_{1}-\varphi_{2}\right) \bar{\psi}_{1}\right)\right) d x d t \\
& \quad+b \iint_{2}\left(u_{1}-u_{2}\right) w_{2} d x d t \\
\leq & C\left(\left\|\varphi_{1}-\varphi_{2}\right\|_{L^{2}(2)}\left(\left\|\psi_{1}\right\|_{L^{2}(2)}+\left\|\psi_{2}\right\|_{L^{2}(2)}\right)+\left\|u_{1}-u_{2}\right\|_{L^{2}(2)}\left\|w_{2}\right\|_{L^{2}(2)}\right) \\
\quad \leq & C_{0}\|f\|_{L^{2}(2)}^{2}, \\
a \iint_{2} & \mathscr{R}\left(\left(\left|\varphi_{1}\right|^{2}-\Lambda\right)\left(\psi_{1}-\psi_{2}\right) \bar{\varphi}_{2}\right) d x d t+b \iint_{2}\left(u_{1}-u_{\mathrm{obs}}\right)\left(w_{1}-w_{2}\right) d x d t \\
\quad \leq C\left(\left\|\left|\varphi_{1}\right|^{2}-\Lambda\right\|_{L^{2}(2)}|| \psi_{1}-\psi_{2}\left\|_{L^{2}(2)}+\right\| u_{1}-u_{\mathrm{obs}}\left\|_{L^{2}(2)}\right\| w_{1}-w_{2} \|_{L^{2}(2)}\right) \\
\leq C_{1}\|f\|_{L^{2}(2)}^{3 / 2} .
\end{array}
$$

From (5.5)-(5.6) we deduce that for $\alpha \geq \alpha_{l}$ such that $\alpha_{l}>C_{0}$ and $\left(\alpha_{l}-C_{0}\right) \min _{f \in \mathscr{K}_{1}}\|f\|_{L^{2}}^{1 / 2}$ $=C_{1}$, we have $\left(P_{g}^{\prime}\left(f_{1}\right)-P_{g}^{\prime}\left(f_{2}\right)\right) \cdot f \geq 0$ and then the convexity of $P_{g}$. In the same way, we can find $\gamma_{l}$ such that for $\gamma \geq \gamma_{l}$ we have the concavity of $Q_{f}$.

We prove now that $P_{g}$ is lower semicontinuous for all $g \in \mathscr{K}_{2}$, and $Q_{f}$ is upper semicontinuous for all $f \in \mathscr{K}_{1}$. Let $f_{k}$ be a minimizing sequence of $J$, that is, $\liminf _{k} J\left(f_{k}, g\right)=$ $\min _{f \in \mathscr{K}_{1}} J(f, g)\left(\forall g \in \mathscr{K}_{2}\right)$. Then $f_{k}$ is uniformly bounded in $\mathscr{K}_{1}$ and we can extract from $f_{k}$ a subsequence also denoted by $f_{k}$ such that $f_{k} \rightarrow f_{g}$ weakly in $\mathscr{K}_{1}$. By using Proposition 5.2, we have then

$$
\mathscr{P}\left(f_{k}, g\right) \longrightarrow\left(\varphi_{g}, u_{g}\right) \text { strongly in } \mathscr{L}^{2}(2) \times \mathbf{L}^{2}(\mathscr{2})
$$

Therefore, since the norm is lower semicontinuous, we have that the map $P_{g}: f \rightarrow J(f, g)$ is lower semicontinuous for all $g \in \mathscr{K}_{2}$. By using the same technique we obtain then $Q_{f}$ is upper semicontinuous for all $f \in \mathscr{K}_{1}$.

In order to obtain the necessary optimality conditions which have been satisfied by the solution of the robust control problem, we introduce the following adjoint problem corresponding to the primal problem (5.1) (we denote by $(\varphi, u)=\mathscr{P}(f, g)$ and $\left(\phi_{1}, U_{1}\right)=$ $(\varphi+\phi, u+U))$ : find $(P, Q) \in \mathscr{E} \times \mathbf{E}_{n}$ such that $\left(\forall(q, v) \in \mathscr{H}^{1}(\Omega) \times V_{n}\right.$ and a.e. $\left.t \in(0, T)\right)$ :

$$
\begin{aligned}
-\eta \int_{\Omega} \rho & \frac{\partial P}{\partial t} q d x-i \eta \kappa \int_{\Omega} \operatorname{div}\left(\rho U_{1}\right) P q d x-i \mu \int_{\Gamma} \rho P q d \Gamma+\int_{\Omega} \rho \bar{B}(u)(P) B(u)(q) d x \\
& +\int_{\Omega} \rho \overline{B(u)\left(\phi_{1}\right)} Q q d x+\int_{\Omega}\left(-\frac{i}{\kappa} \operatorname{div}\left(\rho \overline{\phi_{1}} Q\right)+\rho U_{1} \overline{\phi_{1}} Q\right) q d x \\
& +\int_{\Omega} \rho\left(\left(2\left|\phi_{1}\right|^{2}-1\right) P+\bar{\phi}_{1}^{2} \bar{P}\right) q d x=a \int_{\Omega}\left(|\varphi|^{2}-\Lambda\right) \bar{\varphi} q d x
\end{aligned}
$$




$$
\begin{aligned}
& -\int_{\Omega} \rho \frac{\partial Q}{\partial t} v d x+\int_{\Omega} \rho \operatorname{curl}(Q) \operatorname{curl}(v) d x+\int_{\Omega} \operatorname{div}(Q) \operatorname{div}(\rho q) d x+\int_{\Omega} \rho Q\left|\phi_{1}\right|^{2} v d x \\
& \quad+\int_{\Omega} \rho \mathscr{R}\left(\operatorname{i\eta } \kappa \nabla\left(\phi_{1} P\right)+P B(u)\left(\phi_{1}\right)+\phi_{1} \bar{B}(u)(P)\right) v d x=b \int_{\Omega}\left(u-u_{\mathrm{obs}}\right) v d x, \\
& (P(T), Q(T))=(0,0) .
\end{aligned}
$$

Remark 5.4. (i) The adjoint problem (5.8) is a linear system. By reversing sense of time, that is, $t:=T-t$, and by applying the same way to obtain the result of Theorem 4.2 , we obtain the existence and the uniqueness of $(P, Q)$.

(ii) The adjoint system (5.8) is the weak formulation of the following problem:

$$
\begin{aligned}
& -\eta \rho \frac{\partial P}{\partial t}-i \eta \kappa \operatorname{div}\left(\rho U_{1}\right) P+\bar{B}(u) \cdot(\rho \bar{B}(u)(P))+\rho \overline{B(u)\left(\phi_{1}\right)} Q-\frac{i}{\kappa} \operatorname{div}\left(\rho \overline{\phi_{1}} Q\right)+\rho U_{1} \overline{\phi_{1}} Q \\
& \quad+\rho\left(\left(2\left|\phi_{1}\right|^{2}-1\right) P+\bar{\phi}_{1}^{2} \bar{P}\right)=a\left(|\varphi|^{2}-\Lambda\right) \bar{\varphi}, \\
& -\rho \frac{\partial Q}{\partial t}+\operatorname{curl}(\rho \operatorname{curl}(Q))-\rho \nabla(\operatorname{div}(Q))+\rho Q\left|\phi_{1}\right|^{2} \\
& \quad+\rho \mathscr{R}\left(i \eta \kappa \nabla\left(\phi_{1} P\right)+P B(u)\left(\phi_{1}\right)+\phi_{1} \bar{B}(u)(P)\right)=b\left(u-u_{\mathrm{obs}}\right),
\end{aligned}
$$

subjected to the boundary conditions

$$
\frac{1}{\kappa^{2}} \frac{\partial P}{\partial n}=\mu P, \quad Q \cdot \mathbf{n}=0, \quad \operatorname{curl}(Q)=0, \quad \text { in } \Sigma,
$$

and the final condition

$$
(P(T), Q(T))=(0,0)
$$

We can now give the first-order optimality conditions for the robust control problem $\left(M \mathscr{P}_{1}\right)$.

THEOREM 5.5. Under the assumptions of Theorem 5.3, the optimal solution $\left(f^{*}, g^{*}, u^{*}, \varphi^{*}\right)$ $\in \mathscr{K} \times \mathbf{E}_{n} \times \mathscr{E}$, such that $\left(f^{*}, g^{*}\right)$ is defined by $\left(\mathcal{M}_{1}\right)$ and $\left(\varphi^{*}, u^{*}\right)=\mathscr{P}\left(f^{*}, g^{*}\right)$ solution of (5.1), satisfies

$$
\begin{gathered}
\iint_{2}\left(\rho \operatorname{curl}\left(Q^{*}\right)+\alpha f^{*}\right)\left(f^{*}-f\right) d x d t \geq 0, \\
\iint_{2}\left(\rho \operatorname{curl}\left(Q^{*}\right)-\gamma g^{*}\right)\left(g^{*}-g\right) d x d t \leq 0, \quad \forall(f, g) \in \mathcal{K},
\end{gathered}
$$

where $\left(P^{*}, Q^{*}\right)$ is solution of the adjoint problem (5.8) (corresponding to $\left(\varphi^{*}, u^{*}\right)$ ). 
Proof. The cost function $J$ is a composition of (Fréchet) differentiable maps then $J$ is differentiable and we have $\left(\forall Y=\left(\beta_{1}, \beta_{2}\right) \in \mathscr{K}\right)$

$$
\begin{aligned}
J^{\prime}(f, g) \cdot Y= & a \iint_{2} \mathscr{R}\left(\left(|\varphi|^{2}-\Lambda\right) \bar{\varphi} \psi\right) d x d t+b \iint_{2}\left(u-u_{\mathrm{obs}}\right) w d x d t \\
& +\alpha \iint_{2} f \beta_{1} d x d t-\gamma \iint_{2} g \beta_{2} d x d t
\end{aligned}
$$

where $(\psi, w)=\mathscr{P}^{\prime}(f, g) \cdot Y$ is solution of problem $\left(\mathscr{P}_{F 1}\right)$.

By taking $(q, v)=(P, Q)$ in $\left(\mathscr{P}_{F 1}\right)$, using Green's formula and integrating by time, we obtain (according to the initial condition)

$$
\begin{aligned}
& -\eta \iint_{2} \rho \frac{\partial P}{\partial t} \psi d x d t+\int_{\Omega} \rho P(T) \psi(T) d x-i \eta \kappa \iint_{2} \operatorname{div}\left(\rho U_{1}\right) P \psi d x d t \\
& \quad+i \eta \kappa \iint_{2} \rho w \nabla\left(\phi_{1} P\right) d x d t-i \mu \iint_{\Sigma} \rho P \psi d \Gamma d t+\iint_{2} \rho \bar{B}(u)(P) B(u)(\psi) d x d t \\
& \quad+\iint_{2} \rho \phi_{1} w \bar{B}(u)(P) d x d t+\iint_{2} \rho B(u)\left(\phi_{1}\right) w P d x d t \\
& \quad+\iint_{2} \rho\left(\left(2\left|\phi_{1}\right|^{2}-1\right) P \psi+\phi_{1}^{2} P \bar{\psi}\right) d x d t=0, \\
& -\iint_{2} \rho \frac{\partial Q}{\partial t} w d x d t+\int_{\Omega} \rho Q(T) w(T) d x+\iint_{2} \rho \operatorname{curl}(Q) \operatorname{curl}(w) d x d t \\
& \quad+\iint_{2} \operatorname{div}(Q) \operatorname{div}(\rho w) d x d t+\iint_{2} \rho \Re\left(\bar{\psi} B(u)\left(\phi_{1}\right)\right) Q d x d t \\
& \quad+\iint_{2} \rho\left(\mathscr{R}\left(\overline{\phi_{1}} B(u)(\psi)\right) Q+w\left|\phi_{1}\right|^{2} Q\right) d x d t=\iint_{2} \rho\left(\beta_{1}+\beta_{2}\right) \operatorname{curl}(Q) d x d t .
\end{aligned}
$$

Since $(P, Q)$ is solution of $(5.8)$, we have that

$$
\begin{aligned}
& i \eta \kappa \iint_{2} \rho w \nabla\left(\phi_{1} P\right) d x d t+\iint_{2} \rho \phi_{1} w \bar{B}(u)(P) d x d t+\iint_{2} \rho B(u)\left(\phi_{1}\right) w P d x d t \\
& +\iint_{2} \rho\left(\phi_{1}^{2} P \bar{\psi}-{\overline{\phi_{1}}}^{2} \bar{P} \psi\right) d x d t-\iint_{2} \rho \overline{B(u)\left(\phi_{1}\right)} Q \psi d x d t \\
& -\iint_{2}\left(-\frac{i}{\kappa} \operatorname{div}\left(\rho \overline{\phi_{1}} Q\right)+\rho U_{1} \overline{\phi_{1}} Q\right) \psi d x d t+a \iint_{2}\left(|\varphi|^{2}-\Lambda\right) \bar{\varphi} \psi d x d t=0 \text {, } \\
& \iint_{2} \rho \mathscr{R}\left(\bar{\psi} B(u)\left(\phi_{1}\right)+\overline{\phi_{1}} B(u)(\psi)\right) Q d x d t+b \iint_{2}\left(u-u_{\mathrm{obs}}\right) w d x d t \\
& -\iint_{2} \rho \mathscr{R}\left(i \eta \kappa \nabla\left(\phi_{1} P\right)+P B(u)\left(\phi_{1}\right)+\phi_{1} \bar{B}(u)(P)\right) w d x d t \\
& =\iint_{2} \rho\left(\beta_{1}+\beta_{2}\right) \operatorname{curl}(Q) d x d t \text {. }
\end{aligned}
$$


By adding the real part of the first part of (5.13) and the second part of (5.13), we obtain (since $\mathscr{R}\left(\phi_{1}^{2} P \bar{\psi}-{\overline{\phi_{1}}}^{2} \bar{P} \psi\right)=0$ and $\int_{\Omega}\left(-(i / \kappa) \operatorname{div}\left(\rho \overline{\phi_{1}} Q\right)+\rho U_{1} \overline{\phi_{1}} Q\right) \psi d x=\int_{\Omega} \rho \overline{\phi_{1}} B(u)(\psi) Q d x$, because $Q \cdot \mathbf{n}=0$ )

$$
a \iint_{2} \mathscr{R}\left(\left(|\varphi|^{2}-\Lambda\right) \bar{\varphi} \psi\right) d x d t+b \iint_{2}\left(u-u_{\mathrm{obs}}\right) w d x d t=\iint_{2} \rho\left(\beta_{1}+\beta_{2}\right) \operatorname{curl}(Q) d x d t .
$$

According to the expression of $J^{\prime}(f, g) \cdot Y$, we can deduce that

$$
J^{\prime}(f, g) \cdot Y=\iint_{2}(\rho \operatorname{curl}(Q)+\alpha f) \beta_{1} d x d t+\iint_{2}(\rho \operatorname{curl}(Q)-\gamma g) \beta_{2} d x d t .
$$

Since $\left(f^{*}, g^{*}\right)$ is an optimal solution, we have

$$
\begin{gathered}
\frac{\partial J}{\partial f}\left(f^{*}, g^{*}\right) \cdot\left(f^{*}-f\right) \geq 0, \\
\frac{\partial J}{\partial f}\left(f^{*}, g^{*}\right) \cdot\left(g^{*}-g\right) \leq 0, \quad \forall(f, g) \in \mathscr{K},
\end{gathered}
$$

and then

$$
\begin{gathered}
\iint_{2}\left(\rho \operatorname{curl}\left(Q^{*}\right)+\alpha f^{*}\right)\left(f^{*}-f\right) d x d t \geq 0, \\
\iint_{2}\left(\rho \operatorname{curl}\left(Q^{*}\right)-\gamma g^{*}\right)\left(g^{*}-g\right) d x d t \leq 0, \quad \forall(f, g) \in \mathcal{K} .
\end{gathered}
$$

This completes the proof.

5.1.2. Distributed disturbance in the initial condition of the order parameter. In this section, the disturbance is in $\varphi_{0}$ and the control is in $h$, that is, $\varphi_{0}=g\left(g \in \mathscr{L}_{\infty}^{2}(\Omega)\right), h=$ $f\left(f \in L^{2}(2)\right)$. So the function $(\varphi, u)$ is assumed to be related to the disturbance $f$ and control $g$ through the problem (3.3) (a.e. $t \in(0, T)$ )

$$
\begin{aligned}
& \eta \int_{\Omega} \rho \frac{\partial \varphi}{\partial t} q d x-i \eta \kappa \int_{\Omega} \operatorname{div}(\rho(u+U)) \varphi q d x-i \eta \kappa \int_{\Omega} \operatorname{div}(\rho u) \phi q d x-i \mu \int_{\Gamma} \rho \varphi q d \Gamma \\
& \quad+\int_{\Omega} \rho B(u)(\varphi+\phi) \bar{B}(u)(q) d x+\int_{\Omega} \rho F(\varphi) q d x=\int_{\Omega} \rho B(0)(\phi) \bar{B}(0)(q) d x \\
& \int_{\Omega} \rho \frac{\partial u}{\partial t} v d x+\int_{\Omega} \rho \operatorname{curl}(u) \operatorname{curl}(v) d x+\int_{\Omega} \operatorname{div}(\rho u) \operatorname{div}(v) d x \\
& \quad+\int_{\Omega} \rho \mathscr{R}((\bar{\varphi}+\bar{\phi}) B(u)(\varphi+\phi)) v d x=\int_{\Omega} \rho \mathscr{R}(\bar{\phi} B(0)(\phi)) v d x+\int_{\Omega} \rho f \operatorname{curl}(v) d x \\
& (\varphi(0), u(0))=\left(g, u_{0}\right) .
\end{aligned}
$$

To obtain the regularity of Theorem 4.2 , we suppose that $u_{0} \in \mathbf{L}^{2}(\Omega)$. Let $\mathscr{P}:(f, g) \rightarrow$ $(\varphi, u)=\mathscr{P}(f, g)$ be the map: $L^{2}(2) \times \mathscr{L}_{\infty}^{2}(\Omega) \rightarrow \mathscr{E} \times \mathbf{E}_{n}$ defined by (5.18) and introduce 
the cost function defined by

$$
J(f, g)=\frac{a}{4}\left\||\varphi|^{2}-\Lambda\right\|_{L^{2}(2)}^{2}+\frac{b}{2}\left\|u-u_{\mathrm{obs}}\right\|_{L^{2}(2)}^{2}+\frac{\alpha}{2}\|f\|_{L^{2}(2)}^{2}-\frac{\gamma}{2}\|g\|_{L^{2}}^{2}
$$

where $\alpha, \beta>0, a, b \geq 0$ and $a+b>0$. The functions $\left(u_{\mathrm{obs}}, \Lambda\right) \in \mathbf{L}^{2}(2) \times L^{\infty}(2)$ represent the observation.

In this section, we study the following minimax control problem $\left(M \mathscr{P}_{2}\right)$ :

find an admissible control $f^{*} \in \mathscr{K}_{1}$ and a disturbance $g^{*} \in \mathscr{K}_{2}$ such that $\left(f^{*}, g^{*}\right)$ is a saddle point of the functional $J$ on $\mathscr{K}$, subject to system (5.18),

where, $\mathscr{K}=\mathscr{K}_{1} \times \mathscr{K}_{2}$ such that $\mathscr{K}_{1}$ and $\mathscr{K}_{2}$ are nonempty, closed, convex, bounded subsets of $L^{2}(2)$ and $\mathscr{L}_{\infty}^{2}(\Omega)$, respectively.

The proof of the following propositions and theorem of existence is very similar to that of Propositions 5.1, 5.2 and of Theorem 5.3. So we omit the details.

Proposition 5.6. The function $\mathscr{P}$ is continuously Fréchet differentiable from $L^{2}(2) \times$ $\mathscr{L}_{\infty}^{2}(\Omega)$ to $\mathscr{E} \times \mathbf{E}_{n}$ with the derivative $\mathscr{P}^{\prime}(f, g): Y=\left(\beta_{1}, \beta_{2}\right) \rightarrow(\psi, w)$ given by the linear problem $\left(\mathscr{P}_{F 2}\right)$ (a.e. $t \in(0, T)$ )

$$
\begin{aligned}
& \eta \int_{\Omega} \rho \frac{\partial \psi}{\partial t} q d x-i \eta \kappa \int_{\Omega} \operatorname{div}\left(\rho U_{1}\right) \psi q d x-i \eta \kappa \int_{\Omega} \operatorname{div}(\rho w) \phi_{1} q d x-i \mu \int_{\Gamma} \rho \psi q d \Gamma \\
& \quad+\int_{\Omega} \rho\left(B(u)(\psi)+\phi_{1} w\right) \bar{B}(u)(q) d x+\int_{\Omega} \rho B(u)\left(\phi_{1}\right) w q d x \\
& \quad+\int_{\Omega} \rho\left(\left(2\left|\phi_{1}\right|^{2}-1\right) \psi+\phi_{1}^{2} \bar{\psi}\right) q d x=0, \\
& \int_{\Omega} \rho \frac{\partial w}{\partial t} u d x+\int_{\Omega} \rho \operatorname{curl}(w) \operatorname{curl}(v) d x+\int_{\Omega} \operatorname{div}(\rho w) \operatorname{div}(v) d x+\int_{\Omega} \rho \mathscr{\Re}\left(\bar{\psi} B(u)\left(\phi_{1}\right)\right) v d x \\
& \quad+\int_{\Omega} \rho\left(\Re\left(\overline{\phi_{1}} B(u)(\psi)\right)+w\left|\phi_{1}\right|^{2}\right) v d x=\int_{\Omega} \rho \beta_{1} \operatorname{curl}(v) d x, \\
& (\psi(0), w(0))=\left(\beta_{2}, 0\right),
\end{aligned}
$$

where $\left(U_{1}, \phi_{1}\right)=(u+U, \varphi+\phi)$.

Moreover the following estimates $\left(\forall\left(f_{i}, g_{i}\right) \in L^{2}(2) \times \mathscr{L}_{\infty}^{2}(\Omega), i=1,2\right)$ exist:

(i) $\left\|\mathscr{P}^{\prime}\left(f_{1}, g_{1}\right)\right\|_{\mathscr{L}\left(L^{2}(2) \times L^{2}, \mathscr{E} \times \mathbf{E}_{n}\right)} \leq C_{e}$,

(ii) $\left\|\mathscr{P}^{\prime}\left(f_{1}, g_{1}\right) Y-\mathscr{P}^{\prime}\left(f_{2}, g_{2}\right) Y\right\|_{\mathscr{E} \times \mathbf{E}_{n}}^{2} \leq C_{e}\left(\|X\|_{L^{2}(2) \times L^{2}}\|Y\|_{L^{2}(2) \times L^{2}}^{2}+\|X\|_{L^{2}(2) \times L^{2}}^{2}\|Y\|_{L^{2}(2) \times L^{2}}\right)$, where $f=f_{1}-f_{2}, g=g_{1}-g_{2}$ and $X=(f, g)$.

Proposition 5.7. The map $\mathscr{P}$ defined by (5.18) is continuous from the weak topology of $L^{2}(2) \times \mathscr{L}_{\infty}^{2}(\Omega)$ to the strong topology of $\mathscr{L}^{2}(2) \times \mathbf{L}^{2}(2)$.

Theorem 5.8. For $\alpha$ and $\gamma$ sufficiently large, there exist $\left(f^{*}, g^{*}\right) \in \mathscr{K}$ and $\left(u^{*}, \varphi^{*}\right) \in \mathbf{E}_{n} \times \mathscr{E}$ such that $\left(f^{*}, g^{*}\right)$ is defined by $\left(M \mathscr{P}_{2}\right)$ and $\left(\varphi^{*}, u^{*}\right)=\mathscr{P}\left(f^{*}, g^{*}\right)$ is solution of (5.18). 
Now we establish necessary optimality conditions for the robust control problem $\left(\mathcal{M} \mathscr{P}_{2}\right)$.

Theorem 5.9. Under the assumptions of Theorem 5.8, the optimal solution $\left(f^{*}, g^{*}, u^{*}, \varphi^{*}\right)$ $\in \mathscr{K} \times \mathbf{E}_{n} \times \mathscr{E}$, such that $\left(f^{*}, g^{*}\right)$ is defined by $\left(M \mathscr{P}_{2}\right)$ and $\left(\varphi^{*}, u^{*}\right)=\mathscr{P}\left(f^{*}, g^{*}\right)$ is solution of (5.18), satisfies

$$
\begin{gathered}
\iint_{2}\left(\rho \operatorname{curl}\left(Q^{*}\right)+\alpha f^{*}\right)\left(f^{*}-f\right) d x d t \geq 0, \\
\int_{\Omega} \mathscr{R}\left(\left(\eta \rho P^{*}(0)-\gamma \overline{g^{*}}\right)\left(g^{*}-g\right)\right) d x \leq 0, \quad \forall(f, g) \in \mathscr{K},
\end{gathered}
$$

where $\left(P^{*}, Q^{*}\right)$ is solution of the adjoint problem (5.8) (corresponding to $\left(\varphi^{*}, u^{*}\right)$ ).

Proof. The cost function $J$ is a composition of (Fréchet) differentiable maps, then $J$ is differentiable and we have $\left(\forall Y=\left(\beta_{1}, \beta_{2}\right) \in \mathscr{K}\right)$

$$
\begin{aligned}
J^{\prime}(f, g) \cdot Y= & a \iint_{2} \mathscr{R}\left(\left(|\varphi|^{2}-\Lambda\right) \bar{\varphi} \psi\right) d x d t+b \iint_{2}\left(u-u_{\mathrm{obs}}\right) w d x d t+ \\
& \alpha \iint_{2} f \beta_{1} d x d t-\gamma \int_{\Omega} \mathscr{R}\left(\bar{g} \beta_{2}\right) d x
\end{aligned}
$$

where $(\psi, w)=\mathscr{P}^{\prime}(f, g) \cdot Y$ is solution of problem $\left(\mathscr{P}_{F 2}\right)$.

By taking $(q, v)=(P, Q)$ in $\left(\mathscr{P}_{F 2}\right)$ and integrating by time, we obtain (according to the initial condition)

$$
\begin{aligned}
& -\eta \iint_{2} \rho \frac{\partial P}{\partial t} \psi d x d t+\eta \int_{\Omega} \rho P(T) \psi(T) d x-\eta \int_{\Omega} \rho P(0) \beta_{2} d x-i \eta \kappa \iint_{2} \operatorname{div}\left(\rho U_{1}\right) P \psi d x d t \\
& +i \eta \kappa \iint_{2} \rho w \nabla\left(\phi_{1} P\right) d x d t-i \mu \iint_{\Sigma} \rho \psi P d \Gamma d t+\iint_{2} \rho \bar{B}(u)(P) B(u)(\psi) d x d t \\
& \quad+\iint_{2} \rho \phi_{1} w \bar{B}(u)(P) d x d t+\iint_{2} \rho B(u)\left(\phi_{1}\right) w P d x d t \\
& \quad+\iint_{2} \rho\left(\left(2|\varphi|^{2}-1\right) P \psi+\varphi^{2} P \bar{\psi}\right) d x d t=0, \\
& -\iint_{2} \rho \frac{\partial Q}{\partial t} w d x d t+\int_{\Omega} \rho Q(T) w(T) d x+\iint_{2} \rho \operatorname{curl}(Q) \operatorname{curl}(w) d x d t \\
& \quad+\iint_{2} \operatorname{div}(Q) \operatorname{div}(\rho w) d x d t+\iint_{2} \rho \Re\left(\bar{\psi} B(u)\left(\phi_{1}\right)\right) Q d x d t \\
& \quad+\iint_{2} \rho\left(\mathscr{R}\left(\overline{\phi_{1}} B(u)(\psi)\right) Q+w\left|\phi_{1}\right|^{2} Q\right) d x d t=\iint_{2} \rho \beta_{1} \operatorname{curl}(Q) d x d t .
\end{aligned}
$$


Since $(P, Q)$ is solution of $(5.8)$, we have that

$$
\begin{aligned}
i \eta \kappa \iint_{2} \rho w \nabla\left(\phi_{1} P\right) d x d t+\iint_{2} \rho \phi_{1} w \bar{B}(u)(P) d x d t+\iint_{2} \rho B(u)\left(\phi_{1}\right) w P d x d t \\
\quad+\iint_{2} \rho\left(\phi_{1}^{2} P \bar{\psi}-{\overline{\phi_{1}}}^{2} \bar{P} \psi\right) d x d t-\eta \int_{\Omega} \rho P(0) \beta_{2} d x-\iint_{2} \rho \overline{B(u)\left(\phi_{1}\right)} Q \psi d x d t \\
\quad-\iint_{2}\left(-\frac{i}{\kappa} \operatorname{div}\left(\rho \overline{\phi_{1}} Q\right)+\rho U_{1} \overline{\phi_{1}} Q\right) \psi d x d t+a \iint_{2}\left(|\varphi|^{2}-\Lambda\right) \bar{\varphi} \psi d x d t=0, \\
\iint_{2} \rho \mathscr{R}\left(\bar{\psi} B(u)\left(\phi_{1}\right)\right) Q d x d t+\iint_{2} \rho \mathscr{R}\left(\overline{\phi_{1}} B(u)(\psi)\right) Q d x d t \\
\quad-\iint_{2} \rho \mathscr{R}\left(i \eta \kappa \nabla\left(\phi_{1} P\right)+P B(u)\left(\phi_{1}\right)+\phi_{1} \bar{B}(u)(P)\right) w d x d t \\
\quad+b \iint_{2}\left(u-u_{\mathrm{obs}}\right) w d x d t=\iint_{2} \rho \beta_{1} \operatorname{curl}(Q) d x d t .
\end{aligned}
$$

By adding the real part of the first part of (5.24) and the second part of (5.24), we obtain (by using Green's formula)

$$
\begin{aligned}
& -\eta \int_{\Omega} \rho \mathscr{R}\left(P(0) \beta_{2}\right) d x+a \iint_{2} \mathscr{R}\left(\left(|\varphi|^{2}-\Lambda\right) \bar{\varphi} \psi\right) d x d t+b \iint_{2}\left(u-u_{\mathrm{obs}}\right) w d x d t \\
& \quad=\iint_{2} \rho \beta_{1} \operatorname{curl}(Q) d x d t .
\end{aligned}
$$

According to the expression of $J^{\prime}(f, g) \cdot Y$, we can deduce that

$$
J^{\prime}(f, g) \cdot Y=\iint_{2}(\rho \operatorname{curl}(Q)+\alpha f) \beta_{1} d x d t+\int_{\Omega} \mathscr{R}\left((\eta \rho P(0)-\gamma \bar{g}) \beta_{2}\right) d x
$$

Since $\left(f^{*}, g^{*}\right)$ is an optimal solution, we have then

$$
\begin{gathered}
\iint_{2}\left(\rho \operatorname{curl}\left(Q^{*}\right)+\alpha f^{*}\right)\left(f^{*}-f\right) d x d t \geq 0, \\
\int_{\Omega} \mathscr{R}\left(\left(\eta \rho P^{*}(0)-\gamma \overline{g^{*}}\right)\left(g^{*}-g\right)\right) d x \leq 0, \quad \forall(f, g) \in \mathscr{K} .
\end{gathered}
$$

This completes the proof.

Remark 5.10. In the case where the distributed disturbance is in the initial condition of the potential, we obtain the same results. In this case, the cost functional is given by

$$
J(f, g)=\frac{a}{4}\left\||\varphi|^{2}-\Lambda\right\|_{L^{2}(2)}^{2}+\frac{b}{2}\left\|u-u_{\mathrm{obs}}\right\|_{L^{2}(2)}^{2}+\frac{\alpha}{2}\|f\|_{L^{2}(2)}^{2}-\frac{\gamma}{2}\|g\|_{L^{2}}^{2},
$$

where $\alpha, \gamma>0, a, b \geq 0$ and $a+b>0$. The functions $\left(u_{\mathrm{obs}}, \Lambda\right) \in \mathrm{L}^{2}(2) \times L^{\infty}(2)$ represent the observation.

We can prove also an existence theorem of the robust control problem and obtain necessary optimality conditions for its solution using the same method.

Let $\mathscr{K}=\mathscr{K}_{1} \times \mathscr{K}_{2}$ such that $\mathscr{K}_{1}$ and $\mathscr{K}_{2}$ are nonempty, closed, convex, bounded subsets of $L^{2}(2)$ and $\mathrm{L}^{2}(\Omega)$, respectively. 
For $\alpha$ and $\gamma$ sufficiently large, there exists $\left(f^{*}, g^{*}, u, \varphi\right) \in \mathscr{K} \times \mathbf{E}_{n} \times \mathscr{E}$ satisfying (a.e. $t \in(0, T))$

$$
\begin{aligned}
& \eta \int_{\Omega} \rho \frac{\partial \varphi}{\partial t} q d x-i \eta \kappa \int_{\Omega} \operatorname{div}(\rho(u+U)) \varphi q d x-i \eta \kappa \int_{\Omega} \operatorname{div}(\rho u) \phi q d x-i \mu \int_{\Gamma} \rho \varphi q d \Gamma \\
& \quad+\int_{\Omega} \rho B(u)(\varphi+\phi) \bar{B}(u)(q) d x+\int_{\Omega} \rho F(\varphi) q d x=\int_{\Omega} \rho B(0)(\phi) \bar{B}(0)(q) d x, \\
& \int_{\Omega} \rho \frac{\partial u}{\partial t} v d x+\int_{\Omega} \rho \operatorname{curl}(u) \operatorname{curl}(v) d x+\int_{\Omega} \operatorname{div}(\rho u) \operatorname{div}(v) d x \\
& \quad+\int_{\Omega} \rho \mathscr{R}((\bar{\varphi}+\bar{\phi}) B(u)(\varphi+\phi)) v d x=\int_{\Omega} \rho \mathscr{R}(\bar{\phi} B(0)(\phi)) v d x+\int_{\Omega} \rho f^{*} \operatorname{curl}(v) d x, \\
& (\varphi(0), u(0))=\left(\varphi_{0}, g^{*}\right), \\
& \iint_{2}\left(\rho \operatorname{curl}(Q)+\alpha f^{*}\right)\left(f^{*}-f\right) d x d t \geq 0, \\
& \int_{\Omega}\left(\rho Q(0)-\gamma g^{*}\right)\left(g^{*}-g\right) d x \leq 0, \quad \forall(f, g) \in \mathscr{K},
\end{aligned}
$$

where $(P, Q)$ is solution of (5.8).

5.2. The control in the initial condition of the vector potential. In this section, we formulate the problem in two situations: firstly, the case where the worst disturbance is in the initial condition $\varphi_{0}$, and secondly the case where the disturbance is in the external magnetic field $h$.

5.2.1. Distributed disturbance in the initial condition of the order parameter. We suppose that the control is in $u_{0}$, that is, $u_{0}=f\left(f \in \mathbf{L}^{2}(\Omega)\right)$ and the disturbance is in $\varphi_{0}$, that is, $\varphi_{0}=g\left(g \in \mathscr{L}_{\infty}^{2}(\Omega)\right)$. So the function $(\varphi, u)$ is assumed to be related to the disturbance $f$ and control $g$ through the problem (3.3):

$$
\begin{aligned}
& \eta \int_{\Omega} \rho \frac{\partial \varphi}{\partial t} q d x-i \eta \kappa \int_{\Omega} \operatorname{div}(\rho(u+U)) \varphi q d x-i \eta \kappa \int_{\Omega} \operatorname{div}(\rho u) \phi q d x-i \mu \int_{\Gamma} \rho \varphi q d \Gamma \\
& \quad+\int_{\Omega} \rho B(u)(\varphi+\phi) \bar{B}(u)(q) d x+\int_{\Omega} \rho F(\varphi) q d x \\
& =\int_{\Omega} \rho B(0)(\phi) \bar{B}(0)(q) d x, \quad \text { a.e. } t \in(0, T), \\
& \int_{\Omega} \rho \frac{\partial u}{\partial t} v d x+\int_{\Omega} \rho \operatorname{curl}(u) \operatorname{curl}(v) d x+\int_{\Omega} \operatorname{div}(\rho u) \operatorname{div}(v) d x \\
& \quad+\int_{\Omega} \rho \mathscr{R}((\bar{\varphi}+\bar{\phi}) B(u)(\varphi+\phi)) v d x \\
& =\int_{\Omega} \rho \mathscr{R}(\bar{\phi} B(0)(\phi)) v d x+\int_{\Omega} \rho h \operatorname{curl}(v) d x, \quad \text { a.e. } t \in(0, T), \\
& (\varphi(0), u(0))=(g, f) .
\end{aligned}
$$


To obtain the regularity of Theorem 4.2, we suppose that $h \in L^{2}(2)$. Let $\mathscr{P}:(f, g) \rightarrow$ $(\varphi, u)=\mathscr{P}(f, g)$ be the map: $\mathbf{L}^{2}(\Omega) \times \mathscr{L}_{\infty}^{2}(\Omega) \rightarrow \mathscr{E} \times \mathbf{E}_{n}$ defined by (5.30) and the cost function is defined by

$$
J(f, g)=\frac{a}{4}\left\||\varphi|^{2}-\Lambda\right\|_{L^{2}(2)}^{2}+\frac{b}{2}\left\|u-u_{\mathrm{obs}}\right\|_{L^{2}(2)}^{2}+\frac{\alpha}{2}\|f\|_{L^{2}}^{2}-\frac{\beta}{2}\|g\|_{L^{2}}^{2},
$$

where $\alpha, \gamma>0, a, b \geq 0$, and $a+b>0$. The functions $\left(u_{\mathrm{obs}}, \Lambda\right) \in \mathbf{L}^{2}(2) \times L^{\infty}(2)$ are given.

In this section, we study the following minimax control problem $\left(\mu \mathscr{P}_{3}\right)$ :

find an admissible control $f^{*} \in \mathscr{K}_{1}$ and a disturbance $g^{*} \in \mathscr{K}_{2}$ such that $\left(f^{*}, g^{*}\right)$ is a saddle point of the functional $J$ on $\mathscr{K}$, subject to system (5.30),

where $\mathscr{K}=\mathscr{K}_{1} \times \mathscr{K}_{2}$ such that $\mathscr{K}_{1}$ and $\mathscr{K}_{2}$ are nonempty, closed, convex, bounded subsets of $\mathbf{L}^{2}(\Omega)$ and $\mathscr{L}_{\infty}^{2}(\Omega)$, respectively.

The arguments of Section 5.1 extend directly to the present case without further estimates, so we omit the details. We have then the following results

Proposition 5.11. The function $\mathscr{P}$ is continuously Fréchet differentiable from $\mathbf{L}^{2}(\Omega) \times$ $\mathscr{L}_{\infty}^{2}(\Omega)$ to $\mathscr{E} \times \mathbf{E}_{n}$ with the derivative $\mathscr{P}^{\prime}(f, g): Y=\left(\beta_{1}, \beta_{2}\right) \rightarrow(\psi, w)$ given by the linear problem $\left(\mathscr{P}_{F 3}\right)$ (a.e. $\left.t \in(0, T)\right)$ :

$$
\begin{aligned}
& \eta \int_{\Omega} \rho \frac{\partial \psi}{\partial t} q d x-i \eta \kappa \int_{\Omega} \operatorname{div}\left(\rho U_{1}\right) \psi q d x-i \eta \kappa \int_{\Omega} \operatorname{div}(\rho w) \phi_{1} q d x-i \mu \int_{\Gamma} \rho \psi q d \Gamma \\
& \quad+\int_{\Omega} \rho\left(B(u)(\psi)+\phi_{1} w\right) \bar{B}(u)(q) d x+\int_{\Omega} \rho B(u)\left(\phi_{1}\right) w q d x \\
& \quad+\int_{\Omega} \rho\left(\left(2|\varphi|^{2}-1\right) \psi+\varphi^{2} \bar{\psi}\right) q d x=0, \\
& \int_{\Omega} \rho \frac{\partial w}{\partial t} v d x+\int_{\Omega} \rho \operatorname{curl}(w) \operatorname{curl}(v) d x+\int_{\Omega} \operatorname{div}(\rho w) \operatorname{div}(v) d x+\int_{\Omega} \rho \mathscr{\Re}\left(\bar{\psi} B(u)\left(\phi_{1}\right)\right) v d x \\
& \quad+\int_{\Omega} \rho\left(\mathscr{R}\left(\overline{\phi_{1}} B(u)(\psi)\right)+w\left|\phi_{1}\right|^{2}\right) v d x=0, \\
& (\psi(0), w(0))=\left(\beta_{2}, \beta_{1}\right),
\end{aligned}
$$

where $\left(U_{1}, \phi_{1}\right)=(u+U, \varphi+\phi)$.

Moreover the following estimates $\left(\forall\left(f_{i}, g_{i}\right) \in \mathbf{L}^{2}(\Omega) \times \mathscr{L}_{\infty}^{2}(\Omega), i=1,2\right)$ exist:

(i) $\left\|\mathscr{P}^{\prime}\left(f_{1}, g_{1}\right)\right\| \mathscr{L}\left(\mathbf{L}^{2}(\Omega) \times \mathscr{L}^{2}(\Omega), \mathscr{E} \times \mathbf{E}_{n}\right) \leq C_{e}$,

(ii) $\left\|\mathscr{P}^{\prime}\left(f_{1}, g_{1}\right) Y-\mathscr{P}^{\prime}\left(f_{2}, g_{2}\right) Y\right\|_{\mathscr{E} \times \mathbf{E}_{n}}^{2} \leq C_{e}\left(\|X\|_{L^{2}}\|Y\|_{L^{2}}^{2}+\|X\|_{L^{2}}^{2}\|Y\|_{L^{2}}\right)$,

where $f=f_{1}-f_{2}, g=g_{1}-g_{2}$ and $X=(f, g)$.

Proposition 5.12. The map $\mathscr{P}$ defined by (5.30) is continuous from the weak topology of $\mathbf{L}^{2}(\Omega) \times \mathscr{L}_{\infty}^{2}(\Omega)$ to the strong topology of $\mathscr{L}^{2}(2) \times \mathbf{L}^{2}(2)$.

Theorem 5.13. For $\alpha$ and $\gamma$ sufficiently large (i.e., there exists $\left(\alpha_{l}, \gamma_{l}\right)$ such that $\alpha \geq \alpha_{l}$ and $\left.\gamma \geq \gamma_{l}\right)$, there exist $\left(f^{*}, g^{*}\right) \in \mathscr{K}$ and $\left(\varphi^{*}, u^{*}\right) \in \mathscr{E} \times \mathbf{E}_{n}$ such that $\left(f^{*}, g^{*}\right)$ is defined by $\left(M \mathscr{P}_{3}\right)$ and $\left(\varphi^{*}, u^{*}\right)=\mathscr{P}\left(f^{*}, g^{*}\right)$ is solution of $(5.30)$. 
Next, we establish necessary optimality conditions for the robust control problem $\left(M \mathscr{P}_{3}\right)$.

Theorem 5.14. Under the assumptions of Theorem 3.1, the optimal solution $\left(f^{*}, g^{*}, u^{*}\right.$, $\left.\varphi^{*}\right) \in \mathscr{K} \times \mathbf{E}_{n} \times \mathscr{E}$, such that $\left(f^{*}, g^{*}\right)$ is defined by $\left(\mathcal{M}_{3}\right)$ and $\left(\varphi^{*}, u^{*}\right)=\mathscr{P}\left(f^{*}, g^{*}\right)$ is solution of (5.30), satisfies

$$
\begin{gathered}
\int_{\Omega}\left(\rho Q^{*}(0)+\alpha f^{*}\right)\left(f^{*}-f\right) d x \geq 0, \\
\int_{\Omega} \mathscr{R}\left(\left(\eta \rho P^{*}(0)-\gamma \overline{g^{*}}\right)\left(g^{*}-g\right)\right) d x \leq 0, \quad \forall(f, g) \in \mathscr{K},
\end{gathered}
$$

where $\left(P^{*}, Q^{*}\right)$ is solution of the adjoint problem (5.8) (corresponding to $\left(\varphi^{*}, u^{*}\right)$ ).

Proof. The cost function $J$ is a composition of (Fréchet) differentiable maps, then $J$ is differentiable and we have $\left(\forall Y=\left(\beta_{1}, \beta_{2}\right) \in \mathscr{K}\right)$

$$
\begin{aligned}
J^{\prime}(f, g) \cdot Y= & a \iint_{2} \mathscr{R}\left(\left(|\varphi|^{2}-\Lambda\right) \bar{\varphi} \psi\right) d x d t+b \iint_{2}\left(u-u_{\mathrm{obs}}\right) w d x d t \\
& +\alpha \int_{\Omega} f \beta_{1} d x-\gamma \int_{\Omega} \mathscr{R}\left(\bar{g} \beta_{2}\right) d x,
\end{aligned}
$$

where $(\psi, w)=\mathscr{P}^{\prime}(f, g) \cdot Y$ is solution of problem $\left(\mathscr{P}_{F 3}\right)$.

By taking $(q, v)=(P, Q)$ in $\left(\mathscr{P}_{F 3}\right)$ and integrating by time, we obtain (according to the homogeneous boundary conditions and to the initial condition)

$$
\begin{aligned}
& -\eta \iint_{2} \rho \frac{\partial P}{\partial t} \psi d x d t+\int_{\Omega} \eta \rho P(T) \psi(T) d x-\int_{\Omega} \eta \rho P(0) \beta_{2} d x-i \eta \kappa \iint_{2} \operatorname{div}\left(\rho U_{1}\right) P \psi d x d t \\
& +i \eta \kappa \iint_{2} \rho w \nabla\left(\phi_{1} P\right) d x d t-i \mu \iint_{\Sigma} \rho \psi P d \Gamma d t+\iint_{2} \rho \bar{B}(u)(P) B(u)(\psi) d x d t \\
& \quad+\iint_{2} \rho \phi_{1} w \bar{B}(u)(P) d x d t+\iint_{2} \rho B(u)\left(\phi_{1}\right) w P d x d t \\
& \quad+\iint_{2} \rho\left(\left(2\left|\phi_{1}\right|^{2}-1\right) P \psi+\phi_{1}^{2} P \bar{\psi}\right) d x d t=0, \\
& -\iint_{2} \rho \frac{\partial Q}{\partial t} w d x d t+\int_{\Omega} \rho Q(T) w(T) d x-\int_{\Omega} \rho Q(0) \beta_{1} d x+\iint_{2} \rho \operatorname{curl}(Q) \operatorname{curl}(w) d x d t \\
& \quad+\iint_{2} \operatorname{div}(Q) \operatorname{div}(\rho w) d x d t+\iint_{2} \rho \mathscr{R}\left(\bar{\psi} B(u)\left(\phi_{1}\right)\right) Q d x d t \\
& \quad+\iint_{2} \rho\left(\mathscr{R}\left(\overline{\phi_{1}} B(u)(\psi)\right) Q+w\left|\phi_{1}\right|^{2} Q\right) d x d t=0 .
\end{aligned}
$$


Since $(P, Q)$ is solution of $(5.8)$, we have that

$$
\begin{aligned}
& -\int_{\Omega} \eta \rho P(0) \beta_{2} d x+i \eta \kappa \iint_{2} \rho w \nabla\left(\phi_{1} P\right) d x d t+\iint_{2} \rho \phi_{1} w \bar{B}(u)(P) d x d t \\
& \quad+\iint_{2} \rho B(u)\left(\phi_{1}\right) w P d x d t+\iint_{2} \rho\left(\phi_{1}^{2} P \bar{\psi}-\bar{\phi}_{1}^{2} \bar{P} \psi\right) d x d t-\iint_{2} \rho \overline{B(u)\left(\phi_{1}\right)} Q \psi d x d t \\
& \quad-\iint_{2}\left(-\frac{i}{\kappa} \operatorname{div}\left(\rho \overline{\phi_{1}} Q\right)+\rho U_{1} \overline{\phi_{1}} Q\right) \psi d x d t+a \iint_{2}\left(|\varphi|^{2}-\Lambda\right) \bar{\varphi} \psi d x d t=0 \\
& -\int_{\Omega} \rho Q(0) \beta_{1} d x+\iint_{2} \rho \mathscr{R}\left(\bar{\psi} B(u)\left(\phi_{1}\right)+\overline{\phi_{1}} B(u)(\psi)\right) Q d x d t \\
& \quad-\iint_{2} \rho \mathscr{R}\left(i \eta \kappa \nabla\left(\phi_{1} P\right)+P B(u)\left(\phi_{1}\right)+\phi_{1} \bar{B}(u)(P)\right) w d x d t \\
& \quad+b \iint_{2}\left(u-u_{\mathrm{obs}}\right) w d x d t=0 .
\end{aligned}
$$

By adding the real part of the first part of (5.36) and the second part of (5.36), we obtain

$$
\begin{gathered}
a \iint_{2} \mathscr{R}\left(\left(|\varphi|^{2}-\Lambda\right) \bar{\varphi} \psi\right) d x d t+b \iint_{2}\left(u-u_{\mathrm{obs}}\right) w d x d t \\
=\int_{\Omega} \rho Q(0) \beta_{1} d x+\int_{\Omega} \eta \rho \mathscr{R}\left(P(0) \beta_{2}\right) d x
\end{gathered}
$$

According to the expression of $J^{\prime}(f, g) \cdot Y$, we can deduce that

$$
J^{\prime}(f, g) \cdot Y=\int_{\Omega}(\rho Q(0)+\alpha f) \beta_{1} d x+\int_{\Omega} \mathscr{R}\left((\eta \rho P(0)-\gamma g) \beta_{2}\right) d x
$$

Since $\left(f^{*}, g^{*}\right)$ is an optimal solution we have then

$$
\begin{gathered}
\int_{\Omega}\left(\rho Q^{*}(0)+\alpha f^{*}\right)\left(f^{*}-f\right) d x \geq 0, \\
\int_{\Omega} \mathscr{R}\left(\left(\eta \rho P^{*}(0)-\gamma \overline{g^{*}}\right)\left(g^{*}-g\right)\right) d x \leq 0, \quad \forall(f, g) \in \mathscr{K} .
\end{gathered}
$$

This completes the proof.

5.2.2. Distributed disturbance in the external magnetic field. In this section, the disturbance is in $h$ and the control is in $u_{0}$, that is, $u_{0}=f\left(f \in \mathbf{L}^{2}(\Omega)\right), h=g\left(g \in L^{2}(2)\right)$. So the function $(\varphi, u)$ is assumed to be related to the disturbance $g$ and control $f$ through the problem (3.3) (a.e. $t \in(0, T))$ :

$$
\begin{array}{r}
\eta \int_{\Omega} \rho \frac{\partial \varphi}{\partial t} q d x-i \eta \kappa \int_{\Omega} \operatorname{div}(\rho(u+U)) \varphi q d x-i \eta \kappa \int_{\Omega} \operatorname{div}(\rho u) \phi q d x-i \mu \int_{\Gamma} \rho \varphi q d \Gamma \\
+\int_{\Omega} \rho B(u)(\varphi+\phi) \bar{B}(u)(q) d x+\int_{\Omega} \rho F(\varphi) q d x=\int_{\Omega} \rho B(0)(\phi) \bar{B}(0)(q) d x
\end{array}
$$




$$
\begin{aligned}
& \int_{\Omega} \rho \frac{\partial u}{\partial t} v d x+\int_{\Omega} \rho \operatorname{curl}(u) \operatorname{curl}(v) d x+\int_{\Omega} \operatorname{div}(\rho u) \operatorname{div}(v) d x \\
& \quad+\int_{\Omega} \rho \mathscr{R}((\bar{\varphi}+\bar{\phi}) B(u)(\varphi+\phi)) v d x=\int_{\Omega} \rho \mathscr{R}(\bar{\phi} B(0)(\phi)) v d x+\int_{\Omega} \rho g \operatorname{curl}(v) d x \\
& (\varphi(0), u(0))=\left(\varphi_{0}, f\right) .
\end{aligned}
$$

To obtain the regularity of Theorem 4.2 , we suppose that $\varphi_{0} \in \mathscr{L}_{\infty}^{2}(\Omega)$. Let $\mathscr{P}:(f, g) \rightarrow$ $(\varphi, u)=\mathscr{P}(f, g)$ be the map: $\mathbf{L}^{2}(\Omega) \times L^{2}(2) \rightarrow \mathscr{E} \times \mathbf{E}_{n}$ defined by $(5.40)$ and the cost function is defined by

$$
J(f, g)=\frac{a}{4}\left\||\varphi|^{2}-\Lambda\right\|_{L^{2}(2)}^{2}+\frac{b}{2}\left\|u-u_{\mathrm{obs}}\right\|_{L^{2}(2)}^{2}+\frac{\alpha}{2}\|f\|_{L^{2}}^{2}-\frac{\gamma}{2}\|g\|_{L^{2}(2)}^{2},
$$

where $\alpha, \gamma>0, a, b \geq 0$ and $a+b>0$. The functions $\left(u_{\mathrm{obs}}, \Lambda\right) \in \mathbf{L}^{2}(2) \times L^{\infty}(2)$ are given.

In this section, we study the following minimax control problem $\left(\mathcal{M} \mathscr{P}_{4}\right)$ :

find an admissible control $f^{*} \in \mathscr{K}_{1}$ and a disturbance $g^{*} \in \mathscr{K}_{2}$ such that $\left(f^{*}, g^{*}\right)$ is a saddle point of the functional $J$ on $\mathscr{K}$, subject to system (5.40),

where, $\mathscr{K}=\mathscr{K}_{1} \times \mathscr{K}_{2}$ such that $\mathscr{K}_{1}$ and $\mathscr{K}_{2}$ are nonempty, closed, convex, bounded subsets of $\mathbf{L}^{2}(\Omega)$ and $L^{2}(2)$, respectively.

The proof of the following propositions and theorem of existence is obtained by using similar arguments of Section 5.1, so we omit the details.

Proposition 5.15. The function $\mathscr{P}$ is continuously Fréchet differentiable from $\mathrm{L}^{2}(\Omega) \times$ $L^{2}(2)$ to $\mathscr{E} \times \mathbf{E}_{n}$ with the derivative $\mathscr{P}^{\prime}(f, g): Y=\left(\beta_{1}, \beta_{2}\right) \rightarrow(\psi, w)$ given by the linear problem $\left(P_{F 4}\right)$ (a.e. $\left.t \in(0, T)\right)$ :

$$
\begin{aligned}
& \eta \int_{\Omega} \rho \frac{\partial \psi}{\partial t} q d x-i \eta \kappa \int_{\Omega} \operatorname{div}\left(\rho U_{1}\right) \psi q d x-i \eta \kappa \int_{\Omega} \operatorname{div}(\rho w) \phi_{1} q d x-i \mu \int_{\Gamma} \rho \psi q d \Gamma \\
& \quad+\int_{\Omega} \rho\left(B(u)(\psi)+\phi_{1} w\right) \bar{B}(u)(q) d x+\int_{\Omega} \rho B(u)\left(\phi_{1}\right) w q d x \\
& \quad+\int_{\Omega} \rho\left(\left(2|\varphi|^{2}-1\right) \psi+\varphi^{2} \bar{\psi}\right) q d x=0, \\
& \int_{\Omega} \rho \frac{\partial w}{\partial t} u d x+\int_{\Omega} \rho \operatorname{curl}(w) \operatorname{curl}(v) d x+\int_{\Omega} \operatorname{div}(\rho w) \operatorname{div}(v) d x+\int_{\Omega} \rho \Re\left(\bar{\psi} B(u)\left(\phi_{1}\right)\right) v d x \\
& \quad+\int_{\Omega} \rho\left(\Re\left(\overline{\phi_{1}} B(u)(\psi)\right)+w\left|\phi_{1}\right|^{2}\right) v d x=\int_{\Omega} \rho \beta_{2} \operatorname{curl}(v) d x, \\
& (\psi(0), w(0))=\left(0, \beta_{1}\right),
\end{aligned}
$$

where $\left(U_{1}, \phi_{1}\right)=(u+U, \varphi+\phi)$. 
Moreover the following estimates $\left(\forall\left(f_{i}, g_{i}\right) \in \mathbf{L}^{2}(\Omega) \times L^{2}(2), i=1,2\right)$ exist:

(i) $\left\|\mathscr{P}^{\prime}\left(f_{1}, g_{1}\right)\right\|_{\mathscr{L}\left(\mathbf{L}^{2} \times L^{2}(2), \mathscr{E} \times \mathbf{E}_{n}\right)} \leq C_{e}$,

(ii) $\left\|\mathscr{P}^{\prime}\left(f_{1}, g_{1}\right) Y-\mathscr{P}^{\prime}\left(f_{2}, g_{2}\right) Y\right\|_{\mathscr{E} \times \mathbf{E}_{n}}^{2} \leq C_{e}\left(\|X\|_{\mathbf{L}^{2} \times L^{2}(2)}\|Y\|_{\mathbf{L}^{2} \times L^{2}(2)}^{2}+\|X\|_{\mathbf{L}^{2} \times L^{2}(2)}^{2}\|Y\|_{\mathbf{L}^{2} \times L^{2}(2)}\right)$, where $f=f_{1}-f_{2}, g=g_{1}-g_{2}$ and $X=(f, g)$.

Proposition 5.16. The map $\mathscr{P}$ defined by (5.40) is continuous from the weak topology of $\mathbf{L}^{2}(\Omega) \times L^{2}(2)$ to the strong topology of $\mathscr{L}^{2}(2) \times \mathbf{L}^{2}(2)$.

Theorem 5.17. For $\alpha$ and $\gamma$ sufficiently large, there exist $\left(f^{*}, g^{*}\right) \in \mathscr{Y}$ and $\left(u^{*}, \varphi^{*}\right) \in$ $\mathbf{E}_{n} \times \mathscr{E}$ such that $\left(f^{*}, g^{*}\right)$ is defined by $\left(M \mathscr{P}_{4}\right)$ and $\left(\varphi^{*}, u^{*}\right)=\mathscr{P}\left(f^{*}, g^{*}\right)$ is solution of (5.40).

Next we give necessary optimality conditions for the robust control problem $\left(M \mathscr{P}_{4}\right)$.

Theorem 5.18. Under the assumptions of Theorem 5.8, the optimal solution $\left(f^{*}, g^{*}, u^{*}\right.$, $\left.\varphi^{*}\right) \in \mathscr{K} \times \mathbf{E}_{n} \times \mathscr{E}$, such that $\left(f^{*}, g^{*}\right)$ is defined by $\left(M \mathscr{P}_{4}\right)$ and $\left(\varphi^{*}, u^{*}\right)=\mathscr{P}\left(f^{*}, g^{*}\right)$ is solution of (5.40), satisfies

$$
\begin{gathered}
\int_{\Omega}\left(\rho Q^{*}(0)+\alpha f^{*}\right)\left(f^{*}-f\right) d x \geq 0, \\
\iint_{2}\left(\rho \operatorname{curl}\left(Q^{*}\right)-\gamma g^{*}\right)\left(g^{*}-g\right) d x d t \leq 0, \quad \forall(f, g) \in \mathcal{K},
\end{gathered}
$$

where $\left(P^{*}, Q^{*}\right)$ is solution of the adjoint problem (5.8) (corresponding to $\left(\varphi^{*}, u^{*}\right)$ ).

Proof. The cost function $J$ is a composition of (Fréchet) differentiable maps, then $J$ is differentiable and we have $\left(\forall Y=\left(\beta_{1}, \beta_{2}\right) \in \mathscr{K}\right)$

$$
\begin{aligned}
J^{\prime}(f, g) \cdot Y= & a \iint_{2} \mathscr{R}\left(\left(|\varphi|^{2}-\Lambda\right) \bar{\varphi} \psi\right) d x d t+b \iint_{2}\left(u-u_{\mathrm{obs}}\right) w d x d t \\
& +\alpha \int_{\Omega} f \beta_{1} d x-\gamma \iint_{2} g \beta_{2} d x d t
\end{aligned}
$$

where $(\psi, w)=\mathscr{P}^{\prime}(f, g) \cdot Y$ is solution of problem $\left(\mathscr{P}_{F 4}\right)$.

By taking $(q, v)=(P, Q)$ in $\left(\mathscr{P}_{F 4}\right)$, using Green's formula, and integrating by time, we obtain (according to the homogeneous boundary conditions and to the initial condition)

$$
\begin{aligned}
& -\eta \iint_{2} \rho \frac{\partial P}{\partial t} \psi d x d t+\eta \int_{\Omega} \rho P(T) \psi(T) d x \\
& \quad-i \eta \kappa \iint_{2} \operatorname{div}\left(\rho U_{1}\right) P \psi d x d t+i \eta \kappa \iint_{2} \rho w \nabla\left(\phi_{1} P\right) d x d t \\
& \quad-i \mu \int_{\Gamma} \rho \psi P d \Gamma+\iint_{2} \rho \bar{B}(u)(P) B(u)(\psi) d x d t+\iint_{2} \rho \phi_{1} w \bar{B}(u)(P) d x d t \\
& \quad+\iint_{2} \rho B(u)\left(\phi_{1}\right) w P d x d t+\iint_{2} \rho\left(\left(2\left|\phi_{1}\right|^{2}-1\right) P \psi+\phi_{1}^{2} P \bar{\psi}\right) d x d t=0
\end{aligned}
$$




$$
\begin{aligned}
-\iint_{2} \rho & \frac{\partial Q}{\partial t} w d x d t+\int_{\Omega} \rho Q(T) w(T) d x-\int_{\Omega} \rho Q(0) \beta_{1} d x+\iint_{2} \rho \operatorname{curl}(Q) \operatorname{curl}(w) d x d t \\
& +\iint_{2} \operatorname{div}(Q) \operatorname{div}(\rho w) d x d t+\iint_{2} \rho \mathscr{R}\left(\bar{\psi} B(u)\left(\phi_{1}\right)\right) Q d x d t \\
& +\iint_{2} \rho\left(\mathscr{R}\left(\overline{\phi_{1}} B(u)(\psi)\right) Q+w\left|\phi_{1}\right|^{2} Q\right) d x d t=\iint_{2} \rho \beta_{2} \operatorname{curl}(Q) d x d t
\end{aligned}
$$

Since $(P, Q)$ is solution of (5.8), we have that

$$
\begin{aligned}
i \eta \kappa \iint_{2} \rho w \nabla\left(\phi_{1} P\right) d x d t+\iint_{2} \rho \phi_{1} w \bar{B}(u)(P) d x d t+\iint_{2} \rho B(u)\left(\phi_{1}\right) w P d x d t \\
\quad+\iint_{2} \rho\left(\phi_{1}^{2} P \bar{\psi}-\bar{\phi}_{1}^{2} \bar{P} \psi\right) d x d t-\iint_{2} \rho \overline{B(u)\left(\phi_{1}\right)} Q \psi d x d t \\
\quad-\iint_{2}\left(-\frac{i}{\kappa} \operatorname{div}\left(\rho \overline{\phi_{1}} Q\right)+\rho U_{1} \overline{\phi_{1}} Q\right) \psi d x d t+a \iint_{2}\left(|\varphi|^{2}-\Lambda\right) \bar{\varphi} \psi d x d t=0, \\
-\int_{\Omega} \rho Q(0) \beta_{1} d x+\iint_{2} \rho \mathscr{R}\left(\bar{\psi} B(u)\left(\phi_{1}\right)\right) Q d x d t+\iint_{2} \rho \mathscr{R}\left(\overline{\phi_{1}} B(u)(\psi)\right) Q d x d t \\
\quad-\iint_{2} \rho \mathscr{R}\left(i \eta \kappa \nabla\left(\phi_{1} P\right) d x d t+P B(u)\left(\phi_{1}\right)+\phi_{1} \bar{B}(u)(P)\right) w d x d t \\
\quad+b \iint_{2}\left(u-u_{\mathrm{obs}}\right) w d x d t=\iint_{2} \rho \beta_{2} \operatorname{curl}(Q) d x d t .
\end{aligned}
$$

By adding the real part of the first part of (5.46) and the second part of (5.46), we obtain

$$
\begin{aligned}
& -\int_{\Omega} \rho Q(0) \beta_{1} d x+a \iint_{2} \mathscr{R}\left(\left(|\varphi|^{2}-\Lambda\right) \bar{\varphi} \psi\right) d x d t+b \iint_{2}\left(u-u_{\mathrm{obs}}\right) w d x d t \\
& \quad=\iint_{2} \rho \beta_{2} \operatorname{curl}(Q) d x d t
\end{aligned}
$$

According to the expression of $J^{\prime}(f, g) \cdot Y$, we can deduce that

$$
J^{\prime}(f, g) \cdot Y=\int_{\Omega}(\rho Q(0)+\alpha f) \beta_{1} d x+\iint_{2}(\rho \operatorname{curl}(Q)-\gamma g) \beta_{2} d x d t
$$

Since $\left(f^{*}, g^{*}\right)$ is an optimal solution, we obtain

$$
\begin{gathered}
\int_{\Omega}\left(\rho Q^{*}(0)+\alpha f^{*}\right)\left(f^{*}-f\right) d x \geq 0 \\
\iint_{2}\left(\rho \operatorname{curl}\left(Q^{*}\right)-\gamma g^{*}\right)\left(g^{*}-g\right) d x d t \leq 0, \quad \forall(f, g) \in \mathcal{K} .
\end{gathered}
$$

This completes the proof. 
Remark 5.19. In the case where the distributed disturbance is in the initial condition of the potential, we obtain the same results. In this case the cost functional is given by

$$
J(f, g)=\frac{a}{4}\left\||\varphi|^{2}-\Lambda\right\|_{L^{2}(2)}^{2}+\frac{b}{2}\left\|u-u_{\mathrm{obs}}\right\|_{L^{2}(2)}^{2}+\frac{\alpha}{2}\|f\|_{L^{2}}^{2}-\frac{\gamma}{2}\|g\|_{L^{2}}^{2},
$$

where $\alpha, \gamma>0, a, b \geq 0$ and $a+b>0$.

We can prove also an existence theorem of the robust control problem and obtain necessary optimality conditions for its solution using the same method. Let $\mathscr{K}=\mathscr{K}_{1} \times \mathscr{K}_{2}$ such that $\mathscr{K}_{1}$ and $\mathscr{K}_{2}$ are nonempty, closed, convex, bounded subsets of $\mathbf{L}^{2}(\Omega)$.

For $\alpha$ and $\gamma$ sufficiently large, there exists $\left(f^{*}, g^{*}, \varphi, u\right)$ satisfying

$$
\begin{aligned}
& \eta \int_{\Omega} \rho \frac{\partial \varphi}{\partial t} q d x-i \eta \kappa \int_{\Omega} \operatorname{div}(\rho(u+U)) \varphi q d x-i \eta \kappa \int_{\Omega} \operatorname{div}(\rho u) \phi q d x-i \mu \int_{\Gamma} \rho \varphi P d \Gamma \\
& \quad+\int_{\Omega} \rho B(u)(\varphi+\phi) \bar{B}(u)(q) d x+\int_{\Omega} \rho F(\varphi) q d x \\
& \quad=\int_{\Omega} \rho B(0)(\phi) \bar{B}(0)(q) d x, \quad \text { a.e. } t \in(0, T) \\
& \int_{\Omega} \rho \frac{\partial u}{\partial t} v d x+\int_{\Omega} \rho \operatorname{curl}(u) \operatorname{curl}(v) d x+\int_{\Omega} \operatorname{div}(\rho u) \operatorname{div}(v) d x \\
& \quad+\int_{\Omega} \rho \mathscr{R}((\bar{\varphi}+\bar{\phi}) B(u)(\varphi+\phi)) v d x \\
& \quad=\int_{\Omega} \rho \mathscr{R}(\bar{\phi} B(0)(\phi)) v d x d t+\int_{\Omega} \rho h \operatorname{curl}(v) d x, \quad \text { a.e. } t \in(0, T) \\
& (\varphi(0), u(0))=\left(\varphi_{0}, f^{*}+g^{*}\right), \\
& \int_{\Omega}\left(\rho Q(0)+\alpha f^{*}\right)\left(f^{*}-f\right) d x \geq 0, \\
& \int_{\Omega}\left(\rho Q(0)-\gamma g^{*}\right)\left(g^{*}-g\right) d x \leq 0, \quad \forall(f, g) \in \mathscr{K},
\end{aligned}
$$

where $(P, Q)$ is solution of (5.8).

\section{Conclusion}

We have developed a robust control method for the time-dependent complex GinzburgLandau vortices in superconductivity. This model contains two unknowns, the vector potential $u$ and an order parameter $\varphi$ (or a complex phase-field variable) coming from thermodynamics, where $\varphi$ describes the phase of the underlying superconductivity. The case $|\varphi|$ close to 1 corresponds to the superconducting phase, $|\varphi|$ close to 0 to the normal phase. The existence of weak solution as well as regularity and stability results are established. A robust control problem has been studied for two sets of distributed controls: firstly the control is in the external magnetic field (the disturbance is in the external magnetic field or in the initial condition of the parameter), secondly the control is in the initial condition of the vector potential (the disturbance is in the external magnetic field or in the initial condition of the parameter). Under suitable hypotheses, it is shown that 
one has existence of solution to a corresponding robust control problem, and the appropriate necessary conditions for saddle point optimality are obtained. These conditions are corresponding to identify the gradient of the cost functional that is very useful in the numerical resolution of the robust control problem. For example we can combining the optimal necessary conditions obtained in this paper and the gradient-iterative algorithm to solve the robust control problem numerically (at each iteration $i$, we obtain the numerical approximation $\left(f_{i}, g_{i}\right)$ of the optimal solution $(f, g)$ by $f_{i}=f_{i-1}-\theta J_{f}^{\prime}\left(f_{i-1}, g_{i-1}\right)$ and $g_{i}=g_{i-1}+\omega J_{g}^{\prime}\left(f_{i-1}, g_{i-1}\right)$, where $0<m \leq \theta, \omega \leq M$ are the sequences of step lengths and $m, M$ depending on the second Fréchet derivative of $J$ to ensure the convergence result (see, e.g., Ciarlet [16])).

\section{References}

[1] A. A. Abrikosov, On the magnetic properties of superconductors of the second group, Soviet Physics JETP 5 (1957), no. 6, 1174-1182.

[2] R. A. Adams, Sobolev Spaces, Pure and Applied Mathematics, vol. 65, Academic Press, New York, 1975.

[3] V. Barbu and T. Precupanu, Convexity and Optimization in Banach Spaces, 2nd ed., Mathematics and Its Applications (East European Series), vol. 10, D. Reidel, Dordrecht; Editura Academiei Republicii Socialiste România, Bucharest, 1986.

[4] J. Bardeen, L. N. Cooper, and J. R. Schrieffer, Theory of superconductivity, Physical Review 108 (1957), 1175-1204.

[5] A. Belmiloudi, Analysis of robust control problems for a quasilinear parabolic equations, Proceedings of the 9th IEEE MMAR, "Optimisation of Infinite Dimensional Systems" (Miedzyzdroje, 2003) (S. Domek and R. Kaszynski, eds.), Wydawnictwo Uczelniane Politechniki Szczecińskiej, 2003, pp. 99-108.

[6] - Nonlinear robust control problems of parabolic type equations with time-varying delays given in the integral form, Journal of Dynamical and Control Systems 9 (2003), no. 4, 469-512.

[7] _ Robust control problems associated with time-varying delay nonlinear parabolic equations, IMA Journal of Mathematical Control and Information 20 (2003), no. 3, 305-334.

[8] - Robust and optimal control problems to a phase-field model for the solidification of a binary alloy with a constant temperature, Journal of Dynamical and Control Systems 10 (2004), no. 4, 453-499.

[9] F. Bethuel, H. Brezis, and F. Hélein, Ginzburg-Landau Vortices, Progress in Nonlinear Differential Equations and Their Applications, vol. 13, Birkhäuser Boston, Massachusetts, 1994.

[10] M. W. Cantoni and K. Glover, Gap-metric robustness analysis of linear periodically time-varying feedback systems, SIAM Journal on Control and Optimization 38 (2000), no. 3, 803-822.

[11] S. J. Chapman, A hierarchy of models for type-II superconductors, SIAM Review 42 (2000), no. 4, $555-598$.

[12] S. J. Chapman, J. Rubinstein, and M. Schatzman, A mean-field model of superconducting vortices, European Journal of Applied Mathematics 7 (1996), no. 2, 97-111.

[13] Z. Chen and K.-H. Hoffmann, Numerical studies of a non-stationary Ginzburg-Landau model for superconductivity, Advances in Mathematical Sciences and Applications 5 (1995), no. 2, 363389.

[14] __ Optimal control of dynamical Ginzburg-Landau vortices in superconductivity, Numerical Functional Analysis and Optimization 17 (1996), no. 3-4, 241-258.

[15] Z. Chen, K.-H. Hoffmann, and J. Liang, On a nonstationary Ginzburg-Landau superconductivity model, Mathematical Methods in the Applied Sciences 16 (1993), no. 12, 855-875. 
[16] P. G. Ciarlet, Introduction to Numerical Linear Algebra and Optimisation, Cambridge Texts in Applied Mathematics, Cambridge University Press, Cambridge, 1989.

[17] E. Coskun and M. K. Kwong, Simulating vortex motion in superconducting films with the timedependent Ginzburg-Landau equations, Nonlinearity 10 (1997), no. 3, 579-593.

[18] W. N. Dale and M. C. Smith, Stabilizability and existence of system representations for discretetime time-varying systems, SIAM Journal on Control and Optimization 31 (1993), no. 6, 15381557.

[19] K. Deckelnick, C. M. Elliott, and G. Richardson, Long time asymptotics for forced curvature flow with applications to the motion of a superconducting vortex, Nonlinearity 10 (1997), no. 3, 655678.

[20] Q. Du, Global existence and uniqueness of solutions of the time-dependent Ginzburg-Landau model for superconductivity, Applicable Analysis 53 (1994), no. 1-2, 1-17.

[21] Q. Du and M. D. Gunzburger, A model for superconducting thin films having variable thickness, Applicable Analysis 5 (1992), 363-389.

[22] Q. Du, M. D. Gunzburger, and J. S. Peterson, Analysis and approximation of the Ginzburg-Landau model of superconductivity, SIAM Review 34 (1992), no. 1, 54-81.

[23] I. Ekeland and R. Temam, Convex Analysis and Variational Problems, North-Holland, Amsterdam, 1976.

[24] C. Foias, H. Özbay, and A. Tannenbaum, Robust Control of Infinite-Dimensional Systems. Frequency Domain Methods, Lecture Notes in Control and Information Sciences, vol. 209, Springer, Berlin, 1996.

[25] T. T. Georgiou, Differential stability and robust control of nonlinear systems, Mathematics of Control, Signals, and Systems 6 (1993), no. 4, 289-306.

[26] T. T. Georgiou and M. C. Smith, Robustness analysis of nonlinear feedback systems: an inputoutput approach, IEEE Transactions on Automatic Control 42 (1997), no. 9, 1200-1221.

[27] V. Girault and P.-A. Raviart, Finite Element Methods for Navier-Stokes Equations, Springer Series in Computational Mathematics, vol. 5, Springer, Berlin, 1986.

[28] L. P. Gor'kov and G. M. Eliashberg, Generalization of the Ginzburg-Landau equations for nonstationary problems in the case of alloys with paramagnetic impurities, Soviet Physics JETP 27 (1968), 328-334.

[29] M. Green and D. J. N. Limebeer, Linear Robust Control, Prentice-Hall, New Jersey, 1995.

[30] W. D. Gropp, H. G. Kaper, G. K. Leaf, D. M. Levine, M. Palumbo, and V. M. Vinokur, Numerical simulation of vortex dynamics in type-II superconductors, Journal of Computational Physics 123 (1996), no. 2, 254-266.

[31] C. Hu and R. Temam, Robust control of the Kuramoto-Sivashinsky equation, Dynamics of Continuous, Discrete \& Impulsive Systems. Series B. Applications \& Algorithms 8 (2001), no. 3, 315-338.

[32] O. A. Ladyzenskaya, V. A. Solonnikov, and N. N. Uralceva, Linear and Quasilinear Equations Parabolic Type, Transl. Math. Monographs, vol. 23, American Mathematical Society, Rhode Island, 1968.

[33] J.-L. Lions, Équations différentielles opérationnelles et problèmes aux limites, Die Grundlehren der Mathematischen Wissenschaften, vol. 111, Springer, Berlin, 1961.

[34] J.-L. Lions and E. Magenes, Problèmes aux limites non homogènes et applications. Vol. 1, Travaux et Recherches Mathématiques, no. 17, Dunod, Paris, 1968.

[35] _ Problèmes aux limites non homogènes et applications. Vol. 2, Travaux et Recherches Mathématiques, no. 18, Dunod, Paris, 1968.

[36] B. S. Mordukhovich and K. Zhang, Minimax control of parabolic systems with Dirichlet boundary conditions and state constraints, Applied Mathematics and Optimization 36 (1997), no. 3, 323 360 . 
[37] J. Reinschke, M. W. Cantoni, and M. C. Smith, A robust control framework for linear, timeinvariant, spatially distributed systems, SIAM Journal on Control and Optimization 40 (2001), no. 2, 610-627.

[38] E. Sandier and S. Serfaty, Ginzburg-Landau minimizers near the first critical field have bounded vorticity, Calculus of Variations and Partial Differential Equations 17 (2003), no. 1, 17-28.

[39] Q. Tang and S. Wang, Long time behavior of the Ginzburg-Landau superconductivity equations, Applied Mathematics Letters 8 (1995), no. 2, 31-34.

[40] K. Zhou, J. C. Doyle, and K. Glover, Robust and Optimal Control, Prentice-hall, New Jersey, 1996.

Aziz Belmiloudi: Institut de Recherche Mathématique de Rennes (IRMAR), Université de Rennes1, 35042 Rennes Cedex, France; Center de Maths, Institut National des Sciences, Appliquées (INSA), 20 Avenue des Buttes de Coësmes, CS 14315, 35043 Rennes Cedex, France E-mail address: aziz.belmiloudi@insa-rennes.fr 


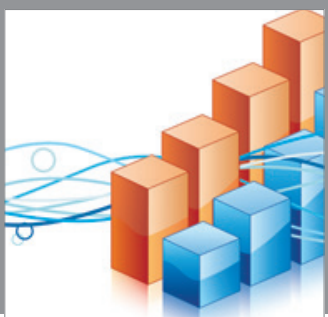

Advances in

Operations Research

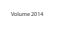

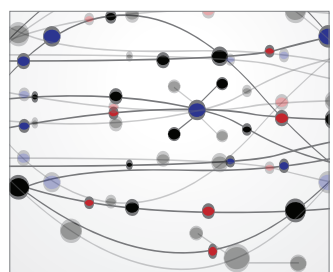

\section{The Scientific} World Journal
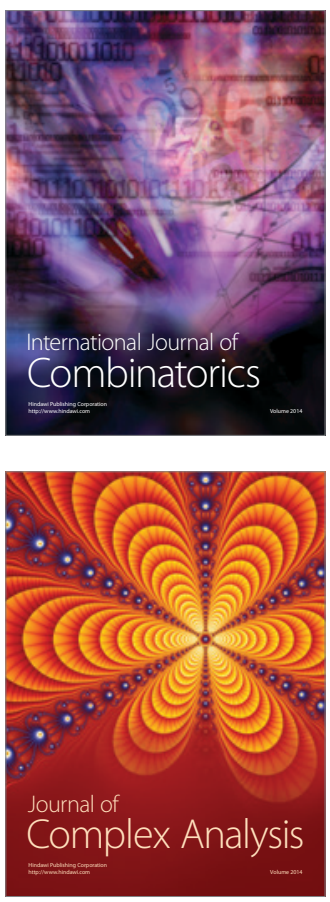

International Journal of

Mathematics and

Mathematical

Sciences
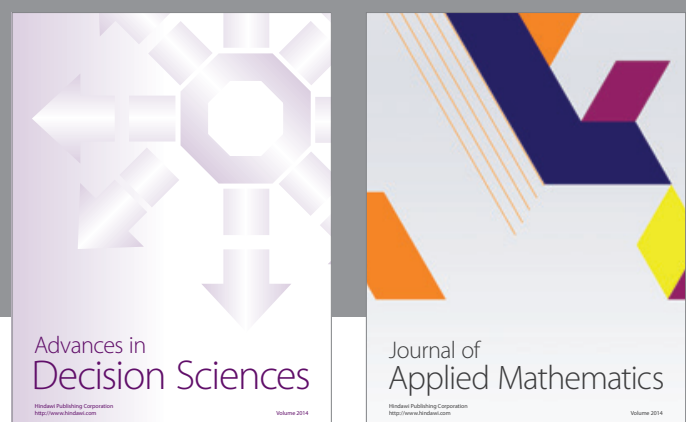

Journal of

Applied Mathematics
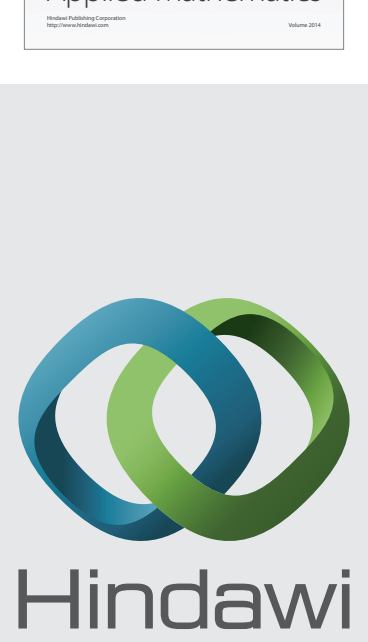

Submit your manuscripts at http://www.hindawi.com
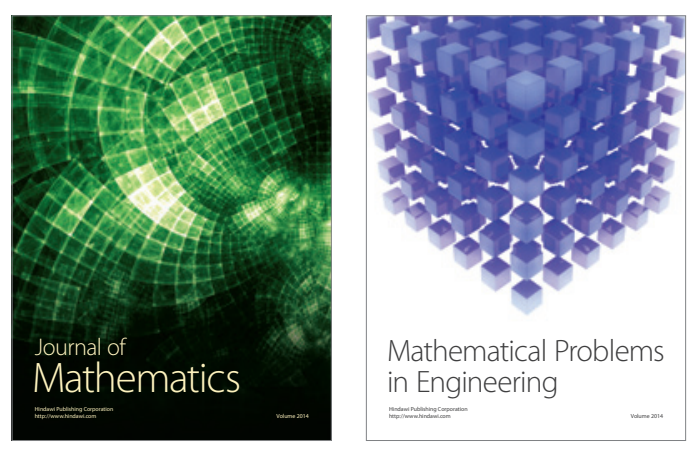

Mathematical Problems in Engineering
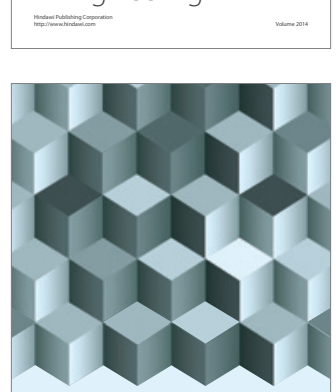

Journal of

Function Spaces
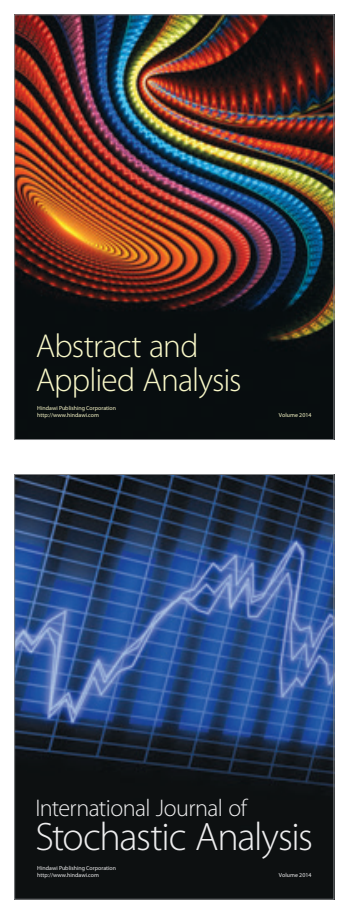

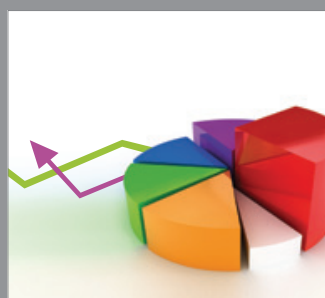

ournal of

Probability and Statistics

Promensencen
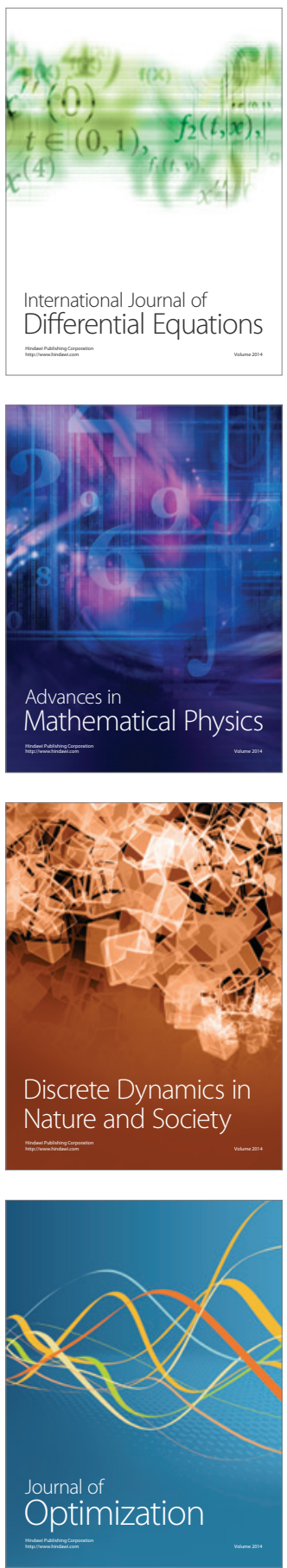